$\mathrm{DE} / \mathrm{ER} / 40444-3$

\title{
Nuclear Structure and Reaction Studies at Medium Energies
}

\author{
Progress Report
}

April 1, 1988 - March 31, 1991

G. W. Hoffmann and R. L. Ray

Department of Physics

The University of Texas at Austin

Austin, Texas 78712

October 1990

\section{PREPARED FOR THE U. S. DEPARTMENT OF ENERGY UNDER GRANT NUMBER DE-FG05-88ER40444 DISCLAIMER}

\footnotetext{
This report was prepared as an accou,.$l$ of work sponsored by an agency of the United States Government. Neither the United States Government nor any agency thereof, nor any of their employees, makes any warranty, express or implied, or assumes any legal liability or responsibility for the accuracy, completeness, or usefulness of any information, apparatus, product, or process disclosed, or represents that its use would not infringe privately owned rights. Reference herein to any specific cummercial product, process, or service by trade name, trademark, manufacturer, or otherwise does not necessarily constitute or imply its endorsement, recommendation, or favoring by the United States Government or any agency thereof. The views and opinions of authors expressed herein do not necessarily state or reflect those of the United States Government or any agency thereof.
} 


\section{Preface}

This document constitutes the 1988 - 1991 technical progress report for Special Research Grant DE-FG05-88ER40444.

The data, calculations, and other infor mation presented here represent work in progress, are preliminary, and should not be quoted without the authors' permission.

Further information relating to the technical portions of this document may be obtained from Dr. G. W. Hoffmann, Department of Physics, The University of Texas at Austin, Austin, Texas 78712, telephone (512) 471-1769 or Bitnet: "HOFFMANN@UTAPHY". 


\begin{abstract}
This document constitutes the $(1988$ - 1991) technical progress report for the ongoing medium energy nuclear physics research program supported by the U. S. Department of Energy through special Research Grant DE-FG05-88ER40444.

The experiments discussed are conducted at the Los Alamos National Laborztory's (LANL) Clinton P. Anderson Meson Physics Facility (LAMPF), the Alternating Gradient Synchrotron (AGS) facility of the Brookhaven National Laboratory (BNL), and at the Fermi National Accelerator Laboratory (FNAL).

The overall motivation for the work discussed in this document is driven by three main objectives: (1) provide hadron-nucleon and hadron-nucleus scattering data which serve to facilitate the study of effective two-body interactions, test (and possibly determine) nuclear structure, and help study reaction mechanisms and dynamics; (2) provide unique, first-of-a-kind "exploratory" hadron-nucleus scattering data in the hope that such data will lead to discovery of new phenomena and new physics; and (3) perform precision tests of fundamental interactions, such as rare decay searches, whose observation would imply fundamental new physics.
\end{abstract}




\section{Contents}

1 Introduction $\quad 6$

2 Nucleon-Nucleon Experiments at LAMPF 7

2.1 EXP 928: Inclusive and Exclusive Quasielastic $\vec{p}+{ }^{2} \mathrm{H}$ Spin Observables at 647 and $800 \mathrm{MeV} \ldots \ldots \ldots 7$

2.2 EXP 1072: $p+p$ Elastic Absolute Cross Sections . . . . . . . . . 11

3 Nucleon-Nucleus Experiments at LAMPF 12

3.1 Proton Elastic Scattering . . . . . . . . . . . . . . . . . . 12

3.1.1 EXP 955U: Polarized Proton Elastic Scattering from ${ }^{12,13} \mathrm{C}$ at $500 \mathrm{MeV} \ldots \ldots \ldots \ldots . \ldots \ldots$

3.1.2 EXP 955: Polarized Proton Elastic Scattering from Polarized ${ }^{13} \mathrm{C} \ldots \ldots \ldots \ldots 17$

3.1.3 EXP 1079: $p+$ A Precision Elastic Forward Angle Cross Sections ...................... 20

3.1.4 EXP 1119: $p+d$ Elastic Absolute Cross Sections . . . . . . 22

3.2 Proton Induced Inelastic Scattering and Reactions . . . . . . . . . . 24

3.2.1 EXP 626: Polarization Transfer for Inclusive Proton Nucleus Inelastic Scattering at $800 \mathrm{MeV}$. . . . . . . . . . . . . 24

3.2.2 EXP 973: Search for Narrow Resonances in the $B=2$ Missing Mass Spectrum from $p+{ }^{3} \mathrm{He}$ Reactions . . . . . . . . . . 27

3.2.3 EXP 1131: Measurement of Polarization Transfer for 800 $\mathrm{MeV}$ Inclusive Proton Scattering at the MRS . . . . . . . 29

3.2.4 EXPS 1052, 1061, 1062: NTOF Calibration and $(p, n)$ Charge Exchange Reactions at Medium Energies . . . . . . . . . 31

4 Pion-Nucleus Experiments at LAMPF

4.1 EXP 1023: Analyzing Powers for Pion Charge Exchange on Polarized ${ }^{13} \mathrm{C} \ldots \ldots \ldots . \ldots . \ldots . \ldots . \ldots 34$

4.2 EXP 1025: Pion Elastic/Inelastic Scattering from Polarized ${ }^{13} \mathrm{C}$ in the Energy Region of the $[3,3]$ Resonance . . . . . . . . . . . . 36

5 Experiments at Brookhaven National Laboratory 39

5.1 BNL EXP 781: Search for Radiative Transitions in the Hypernucleus ${ }_{\Lambda}^{10} \mathrm{~B} \ldots \ldots \ldots \ldots$

5.2 BNL EXP 791: Search for $K_{L}^{0} \rightarrow \mu e, K_{L}^{0} \rightarrow e e \ldots \ldots . \ldots 1$

6 Experiments at Fermilab $\quad \mathbf{4 3}$

6.1 FNAL EXP 772: Study of the Nuclear Antiquark Sea Via $p+N \rightarrow$ Dimuons . . . . . . . . . . . . . . . . . . 43

7 Polarized Nuclear Target Development Work 46 
8 Theoretical Work.

8.1 Spin-Flip Cross Sections for $\left.{ }^{13} \mathrm{C}(\vec{p}, \vec{n})\right)^{13} \mathrm{~N}(\mathrm{~g} . \mathrm{s})$ at $500 \mathrm{MeV} \ldots \ldots . .48$

8.2 Multiple Scattering Description of Intermediate Energy DeuteronNucleus Scattering . . . . . . . . . . . . . . . . . . 50

8.3 Coupled-Channels Dirac Equation Treatment of Large Angle $\vec{p}+{ }^{40} \mathrm{Ca}$ at $500 \mathrm{MeV} \ldots \ldots \ldots \ldots 5$

8.4 Antisymmetrization Correction for Nucleon-Nucleus Elastic Scattering 57

8.5 Spin-One Kemmer-Duffin-Petiau Equation and Intermediate-Evergy Deuteron-Nucleus Scattering . . . . . . . . . . . . . . . . . . . . . 59

8.6 Effective Interactions for Nucleon-Nucleus Scattering Above $300 \mathrm{MeV} 61$

8.7 Analyzing Powers for $\vec{p}+{ }^{208} \mathrm{~Pb}$ at Large Momentum Transfers . . . 66

8.8 Comparison of Dirac and Schrödinger Descriptions of Spin Observables for Proton-Nucleus Elastic Scattering at 650 and $800 \mathrm{MeV}$. . 68

8.9 Nonrelativistic Density-Dependent Calculations for $\vec{p}+{ }^{13} \overrightarrow{\mathrm{C}} \ldots \ldots$

9 Publications (1988 - 1990) $\quad 73$

9.1 Journal and Conference Proceedings in Print . . . . . . . . . . . T3

9.2 Journal Articles Submitted for Publication . . . . . . . . . . . . . 80

9.3 Abstracts ...................... 82

10 Copies of Publications (1990 - 1991) 85 


\section{Introduction}

This document is a progress report for research projects in medium energy nuclear physics (MEP) supported by the U. S. Department of Energy's Three-Year Grant No. DE-FG05-88ER40444 with the University of Texas at Austin. University of Texas personnel associated with these projects for the 1988-1991 period (not inclusive) are G. W. Hoffmann (Professor); R. L. Ray, M. L. Barlett, and G. Pauletta (Research Scientists), J. McDonough (post-doc); D. E. Ciskowski, W. F. Kielhorn, M. J. Purcell, D. E. Read, S. D. Worm, A. R. Smith (Graduate Students), and S. Z. Mujtaba (undergraduate student). Dr. Ciskowski received his Ph.D in 1989, while W. Kielhorn and M. Purcell should receive their Ph.D's in January 1991. Dr. Pauletta left our group to take a tenured faculty position at The University of Udine in Italy. Dr. Barlett is now a staff member at the University of Texas's Applied Research Laboratory at the Balcones Research Center.

The overall motivation for our MEP research program is driven by three main objectives. The first is to provide hadron-nucleon and hadron-nucleus scattering data which serve to facilitate the study of effective two-body interactions, test (and possibly determine) nuclear structure, and help study reaction mechanisms and dynamics. We have adopted the pragmatic philosophy that experiment and theory must merge in this effort in order that significant progress be made. For this reason substantial effort has gone into providing strong theoretical support of the experimental program. Another objective of the program is to provide unique, first-of-a-kind, "exploratory" hadron-nucleus scattering data in the hope that such data will lead to discovery of new phenomena and new physics and stimulate further theoretical work. Most of the above research is associated with experiments done at the Los Alamos Clinton P. Anderson Meson Physics Facility (LAMPF).

Finally, with the realization that the study of fundamental interactions has come to be regarded as forming a vital branch of research in medium energy physics, we have expanded our program to include experiments which provide stringent tests of the Minimal Standard Model (MSM) and in this way probe for physics beyond the Standard Model. Specifically our work in this area involves an experiment at the Brookhaven National Laboratory which searches for previously unobserved and "forbidden" neutral kaon decay.

Our group has authored 43 refereed journal articles, 16 published conference proceedings, and 25 abstracts during the 1988-1991 research period.

The material contained in this document summarizes the highlights of our 1988-1991 efforts with emphasis given to the more recent (1990-1991) work. 


\section{Nucleon-Nucleon Experiments at LAMPF}

\subsection{EXP 928: Inclusive and Exclusive Quasielastic $\vec{p}+{ }^{2} \mathrm{H}$ Spin Observables at 647 and $800 \mathrm{MeV}$}

(M. L. Barlett, G. W. Hoffmann, L. Ray, G. Pauletta; LANL; Rutgers;

UCLA; M. L. Barlett, G. W. Hoifmann, J. B. McClelland, spokesmen)

A longstanding goal of the nucleon-nucleon (NN) program at LAMPF has been to provide sufficient data to allow for an accurate determination of the $I=0,1$ phases up to an energy of $1 \mathrm{GeV}$ (laboratory). Presently, the $\mathrm{I}=1$ phases are fairly well-determined, but the $I=0$ phases are stili uncertain above $500 \mathrm{MeV}$.

Over the years we have performed a number of quasielastic experiments on deuterium in order to provide quasi-free $p n$ data to aid the phase shift solutions, particularly at small momentum transfers $\left(\mathrm{q} \leq 2 \mathrm{fm}^{-1}\right)$. In EXP 928 inclusive and exclusive $p p$ and $p n$ quasielastic data for $\vec{p}+{ }^{2} \mathrm{H}$ were taken at 647 and $800 \mathrm{MeV}$. The experiment measured analyzing powers $\left(A_{y}\right)$ and spin-rotation depolarization parameters $\left(D_{i j}\right)$. The results are discussed in detail in Ref. 1. The experiment was done at the external proton beam (EPB) area at LAMPF using a liquid deuterium target, the SCYLLA low-resolution spectrometer, and the JANUS polarimeter. A recoil particle detector system allowed exclusive measurements to be made.

The exclusive data were compared with previous, free NN scattering data and with phase shift predictions ${ }^{2}$ and were found to be in very good agreement with each. The inclusive data, shown in Figs. 1-3, are well described by a simple isospin weighting model. This model assumes that the inclusive quasielastic spindependent differential cross sections are proportional to the sum of the exclusive $p p$ and $p n$ quasielastic spin-dependent differential cross sections. From this it follows that the inclusive quasielastic spin observable $X_{I}$ can be expressed as

$$
X_{I}=\frac{\sigma_{p p}^{e x}}{\sigma_{p p}^{e x}+\sigma_{p n}^{e x}} X_{p p}^{e x}+\frac{\sigma_{p n}^{e x}}{\sigma_{p p}^{e x}+\sigma_{p n}^{e x}} X_{p n}^{e x},
$$

where $\sigma_{p p}^{e x}\left(\sigma_{p n}^{e x}\right)$ is the exclusive $p p(p n)$ differential cross section at the appropriate angle and $X_{p p}^{e x}\left(X_{p n}^{e x}\right)$ is the exclusive quasielastic $p p(p n)$ spin observable corresponding to $X_{I}$. One can see that if $X_{I}, X_{p p}^{e x}$, and $\sigma_{p p}^{e x} / \sigma_{p n}^{e x}$ are known, then $X_{p n}^{e x}$ may be calculated. The model was tested by using the above equation together with SP87 NN observables ${ }^{2}$ to predict the $\vec{p}+{ }^{2} \mathrm{H}$ inclusive quasielastic analyzing power and $D_{i j}$. The results are shown in Figs. 1-3 by the solid curves which are seen to be in very good agreement with the data.

Based on this success the model was then used to extract exclusive $p n D_{i j}$ observables from the inclusive data. The results at 800 and $647 \mathrm{MeV}$ are shown in Figs. 4 and 5, respectively, in comparison with previous data at $800 \mathrm{MeV}$ at forward angles (crosses) and with SP87 phase shift predictions (solid curves). ${ }^{2}$ These $p n$ 
observables are consistent with previous data but differ quantitatively from the phase shift predictions, particularly $D_{N N}$ and $D_{L S}$. At $647 \mathrm{MeV}$ the phase shift analysis ${ }^{2}$ reproduces the overall magnitudes of the deduced observables. These results lend support to the belief that the $I=0 \mathrm{NN}$ phases above $500 \mathrm{MeV}$ need improvement.

In addition to providing new data and testing the quasielastic method this work shows that the isospin weighting model provides an expedient means for studying $p n$ elastic scattering in that only one-arm, inclusive quasielastic measurements are needed in order to extract $p n$ observables. Such work is vital to the NN program as long as an insufficient amount of free $p n$ data exist.

\section{References}

[1] M. L. Barlett et al., Phys. Rev. C 40, 2697 (1989).

[2] R. A. Arndt et al., Phys. Rev. D 35, 128 (1987).

\section{Figure Captions}

Figure 1. The 800 and $647 \mathrm{MeV}$ inclusive analyzing power for $\vec{p}+{ }^{2} \mathrm{H}$. The curves are from the isospin weighting model.

Figure 2. The $800 \mathrm{MeV}$ inclusive spin-rotation-depolarization parameters for $\vec{p}+{ }^{2} \mathrm{H}$. The curves are from the isospin weighting model.

Figure 3. Same as Fig. 2 except $647 \mathrm{MeV}$.

Figure 4. The $800 \mathrm{MeV}$ exclusive $(p n)$ spin-rotation-depolarization parameters deduced using the isospin weighting model are compared with previous data (crosses) and predictions of phase shift analysis.

Figure 5. Same as Fig. 4 except $647 \mathrm{MeV}$. 
Figure 1
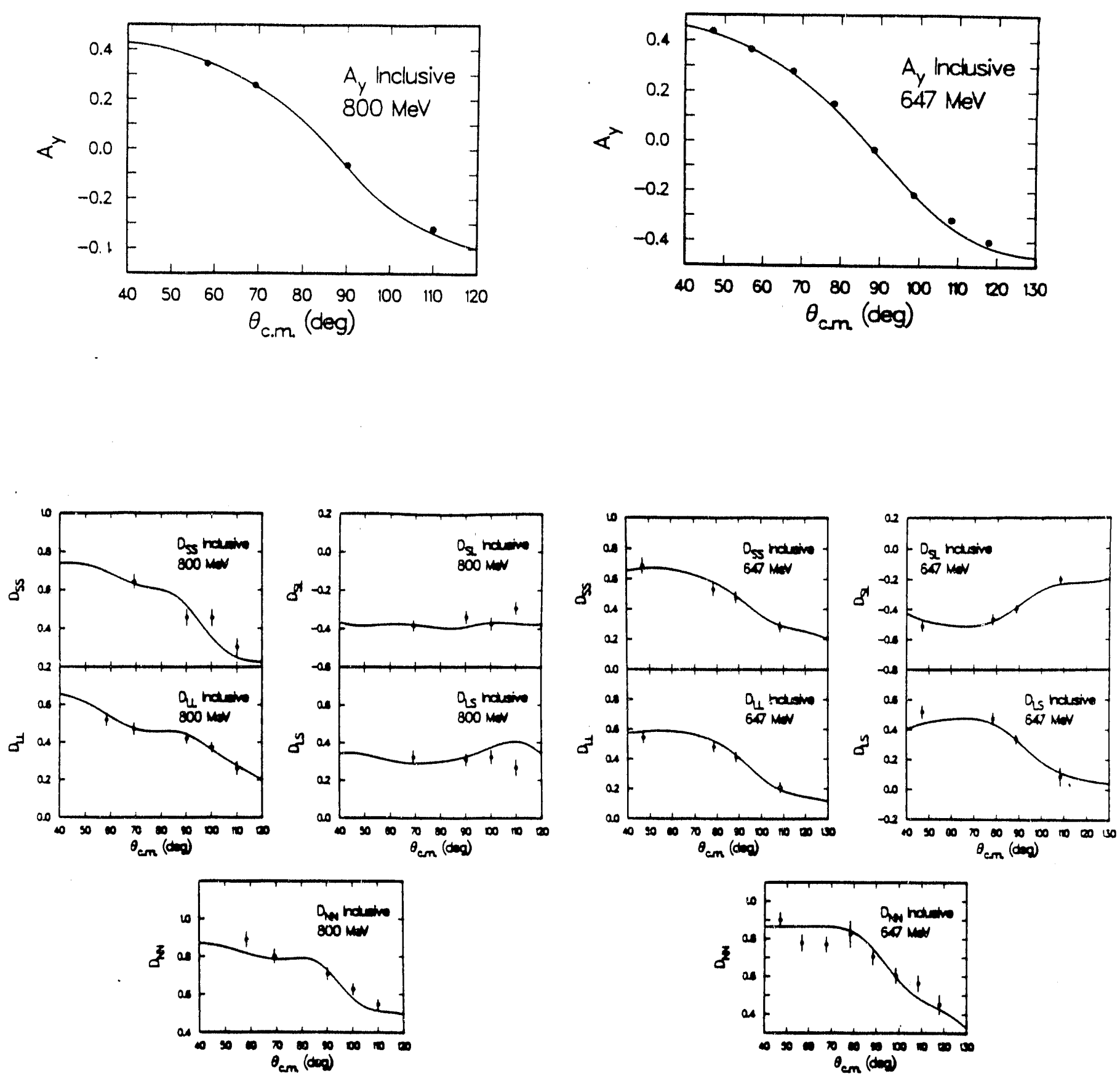

Figure 2

Figure 3 
Figure 4
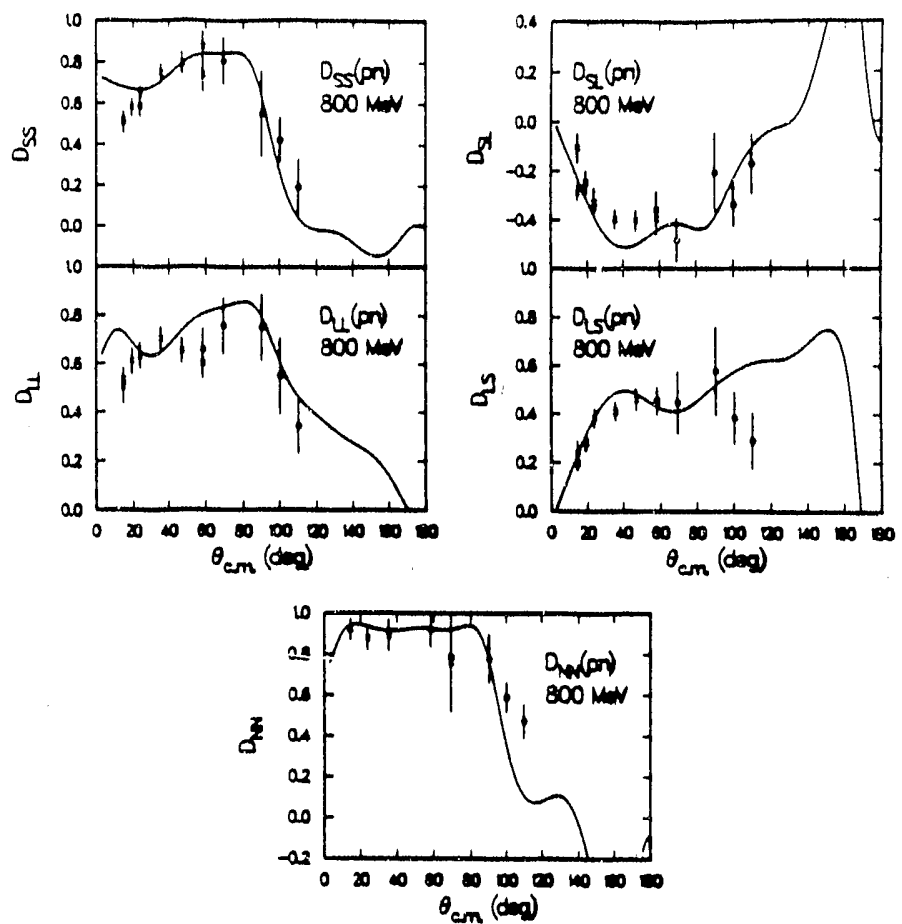

Figure 5
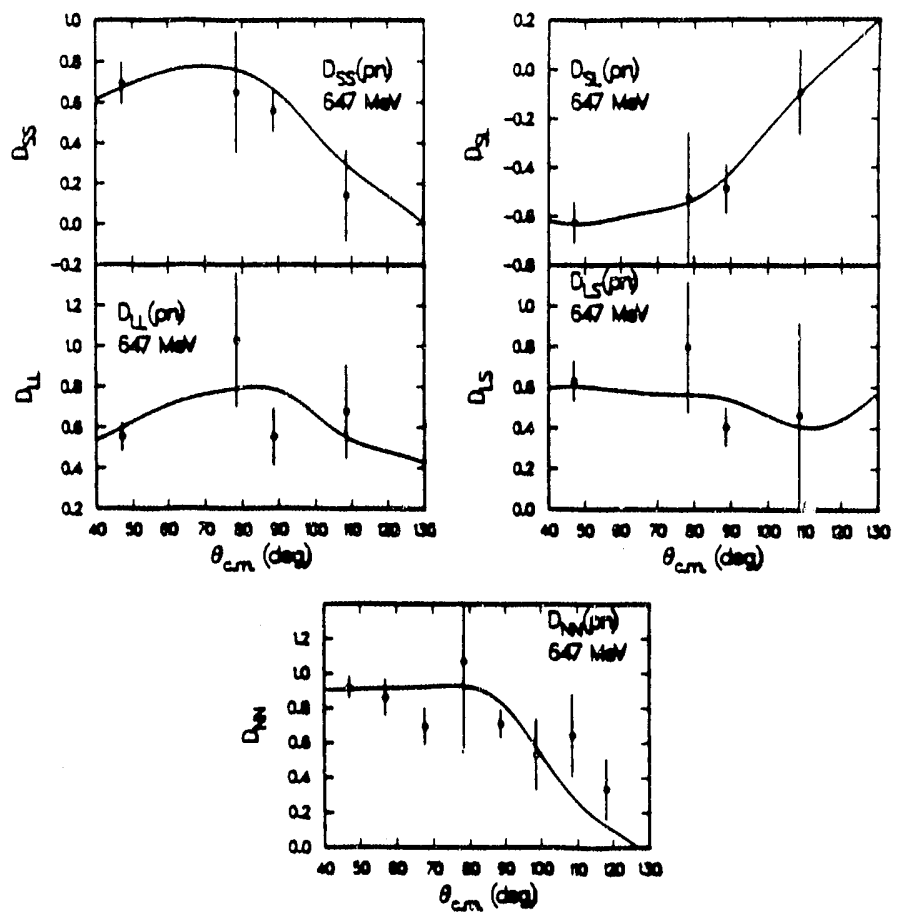


\subsection{EXP 2072: $p+p$ Elastic Absolute Cross Sections}

(M. L. Barlett; LANL; UCLA; Rice; Rutgers; Texas A\&M;

M. McNaughton, spokesman)

One objective of the nucleon-nucleon program at LAMPF is to provide a complete set of elastic data for the $p+p$ and $n+p$ systems between laboratory energies 500 $800 \mathrm{MeV}$. The goal is that these data be used to determine the nucleon-nucleon $(\mathrm{NN})$ phase shifts through careful phase-shift analysis.

Over the past 15 years most of the experimental effort for $p+p$ has gone into "spin-observable" measurements, and little attention has been given to providing the best possible cross section data. The typical quoted errors for the "best" $p+p$ cross sections are $\pm(5-10) \%$. However, it is bilinear combinations of the scattering amplitudes which are equal to products of spin-observables and the differential cross sections that are fit in the phase shift analyses. An example is the expression for the spin-depolarization parameter $D_{N N}$ :

$$
\frac{d \sigma}{d \Omega}(\theta) D_{N N}(\theta)=\frac{1}{2}\left[|a|^{2}+|b|^{2}-|c|^{2}-|d|^{2}+|e|^{2}+|f|^{2}\right],
$$

where $a, b, c, d, e, f$ are the $p+p$ scattering amplitudes as defined by Bystricky and Lehar ${ }^{1}$. Thus, knowledge of the $p+p$ amplitudes may be limited by the accuracy of the available cross section data and not that of the spin-observable data. The elastic cross section data also provide important constraints on inelasticities through the reaction cross section. Additionally, more accurate $p+p$ cross section data are desireable because many intermediate energy proton-nucleus experiments have established absolute normalizations with respect to measured $p+p$ elastic differential cross sections.

Driven by the above motivation, the experiment measured absolute differential cross sections over the center-of-momentum angular range $15^{\circ}-90^{\circ}$ at incident laboratory energies $500,580,650,733$, and $800 \mathrm{MeV}$. A novel bearn counting technique was developed to count beam particles individually with the $5 \mathrm{~ns}$ LAMPF accelerator microstructure. ${ }^{2}$ The final state protons were detected in coincidence, with position and angle information recorded for both protons. Redundant sets of wire chambers (a total of $10 \mathrm{XY}$ pairs) and four banks of scintillators provided overall detector efficiency greater than $99 \%$. Data analysis is still underway, but preliminary results indicate that the differential cross sections are determined to $\pm 1 \%$.

\section{References}

[1] J. Bystricky, F. Lehar, and P. Winternitz, J. de Phys. 39, 1 (1978).

[2] E. Gülmez and C. A. Whitten, Jr., Nucl. Instr. and Methd. (in press); E. Gülmez et al., Nucl. Instr. and Methd. (in press). 


\section{Nucleon-Nucleus Experiments at LAMPF}

\subsection{Proton Elastic Scattering}

\subsubsection{EXP 955U: Polarized Proton Elastic Scattering from ${ }^{12,13} \mathrm{C}$ at 500 $\mathrm{MeV}$}

(G. W. Hoffmann, Mr. L. Barlett, D. Ciskowski, G. Pauletta, M. Purcell, L. Ray; LANL; Minnesota; ASU; OSU;

G. W. Hoffmann, M. L. Barlett, spokesmen)

For more than a decade, experiments with proton bearns have provided a variety of data which have been used to test models of nucleon + nucleus elastic scattering at intermediate energies. Analyzing powers, $A_{y}$, and spin-rotation-depolarization observables, $D_{i j}$, measured with polarized beams have been particularly useful in guiding theoretical development. Even today, the study of spin dependence in the scattering process continues to be an important part of me.ium energy nuclear physics research. Indeed, during the last few years much work has gone into development of polarized nuclear targets for use in scattering experiments. The intent is clearly to introduce a new degree of freedom, that of target polarization, into the scattering problem. For example, the first phase of EXP $955, \vec{p}+{ }^{13} \overrightarrow{\mathrm{C}}$ at $497.5 \mathrm{MeV}$ has provided $A_{\text {ooon }}$ and $A_{\text {oonn }}$ data. In order to analyze these new data, it is necessary to measure the other scattering observables from both the ${ }^{12} \mathrm{C}$ core and ${ }^{13} \mathrm{C}$ at the same energy. The $\vec{p}+{ }^{12} \mathrm{C}$ data enable determination of phenomenological representations of the twelve nucleon "core" scattering amplitudes and distorted waves that are required in distorted wave Born approximation (DWBA) calculations for $\vec{p}+{ }^{13} \overrightarrow{\mathrm{C}}$ elastic scattering. The measured ratio of the ${ }^{13} \mathrm{C}$ to ${ }^{12} \mathrm{C}$ differential cross sections can be used to constrain models of the ${ }^{13} \mathrm{C}$ valence neutron wave function.

A complete set of $\vec{p}+{ }^{12} \mathrm{C}$ elastic scattering measurements is interesting for other reasons. Such data provide a means for testing certain aspects of relativistic and nonrelativistic models of the scattering process, as well as for studying medium effects, nuclear structure models, and multistep processes. They also allow empirical determination of important components of the nucleon-nucleon (NN) effective interaction. Similar conments apply for $\vec{p}+{ }^{13} \mathrm{C}$. The $\vec{p}+{ }^{13} \mathrm{C} d \sigma / d \Omega$ and $A_{y}$ data also serve as benchmarks for the more difficult $\vec{p}+{ }^{13} \overrightarrow{\mathrm{C}}$ experiment. Studies of $D_{N N}$ focus attention on important $\Delta J=1$ contributions to the $\vec{p}+{ }^{13} \mathrm{C}$ elastic scattering amplitude. When these transitions occur, $D_{N N}$ becomes less than unity, and the spin-flip probability, given by $(1 / 2)\left(1-D_{V N}\right)$, is non-zero.

The data ${ }^{1}$ were taken at LAMPF with the high resolution spectrometer (HRS) and its focal plane polarimeter (FPP). The data (and curves to be discussed) are shown in Figs. 1-3. Except at the largest angles, statistical errors are typically $\pm 1 \%$ for the cross sections $\pm(0.01-0.02)$ for $A_{y}$, and $\pm(0.03-0.05)$ for $D_{i j}$. For 
the $d \sigma / d \Omega$ and $A_{y}$ data the systematic errors are small $\leq \pm 2 \%$; for the $D_{1}$, data. the systematic uncertainties in $D_{s L}$ and $D_{S S}$ are $\leq \pm 0.02$, while those for $D_{V . v}$ are $\leq \pm 0.04$. The ${ }^{13} \mathrm{C} /{ }^{12} \mathrm{C}$ differential cross section ratios versus momentum transfer are plotted in Fig. 4, along with curves to be discussed.

In Figs. 1.3 the dashed curves (standard RIA-DWBA) are results from the relativistic impulse approyimation (RIA) - Dirac equation model for ${ }^{12} \mathrm{C}$ and the relativistic DWBA model for ${ }^{13} \mathrm{C}$ (Ref. 2). The solid curves result from a Dirac optical model fit to the $\vec{p}+{ }^{12} \mathrm{C}$ data and a $p+{ }^{13} \mathrm{C}$ relativistic DWBA calculation that uses the zcattering amplitudes and distorted was s from the optical model fit. The improvement over the standard RIA.DWBA prediction is a direct consequence of using a phenomenological model which accurately reproduces the $\vec{p}+{ }^{12} \mathrm{C}$ data. To observe effects due to the $13^{\text {th }}$ nucleon, with protnn elastic scattering from an unpolarized ${ }^{13} \mathrm{C}$ target. the quantity $D_{V N}$ and the small differences between the ${ }^{13} \mathrm{C}$ and ${ }^{1 ?}$ " observables. must be studied. The arnount by which $D_{. v}$ differs from un: $\cdots$ is determined by a few of the $\Delta J=i$ components of the $N . \vee$ effective interaction alorg with the valence nucleon wave function. The ${ }^{13} \mathrm{C} /{ }^{12} \mathrm{C}$ cross section ratio primarily depeads upon the $\lrcorner J=0$ componens of the $N \mathrm{~N}$ amplitudes and is particularly sensitive to he large. upper component of the valence nucleon wave fu. iction. In Fig. 16 of Ref. 2, a variety of RIA-DWBA $D_{N N}$ predictions are shown for $500.4 \vee \vec{p}+{ }^{13} \mathrm{C}$. T wo of these re cornpared with the new data in the upper half of Fig. 3; tne standard RIA-DWBA result is indicated by the dashed curve: the solid curve also represents a RIA-DWBA result, except that the pseudovector form of the NN invariant amplitude was used, and the isoscalar three-vector current was suppressed. ${ }^{2}$ The two curves shown in the lower half of the figure correspond to those in the upper half, except that the phenomenological ${ }^{12} \mathrm{C}$ distorted waves and scattering amplitudes were used instead. None of the four results fit the data very well. In Fig. 4, the ${ }^{13} \mathrm{C} /{ }^{12} \mathrm{C} d \sigma / d \Omega$ ratio is compared with two model predictions. The ratio, $\left({ }^{13} \mathrm{C}\right.$ relativistic DWBA $d \sigma / d \Omega$ using phenomenological distorted waves and core amplitudes)/(the Dirac phenomenological ${ }^{12} \mathrm{C} d \sigma / d \Omega$ ) is indicated by the solid line. The dashed curve results from using the Dirac optical potential to calculate both the ${ }^{12} \mathrm{C}$ and ${ }^{13} \mathrm{C}$ cross sections, where in both cases the radii were scaled by $A^{1 / 3}$. The majority of the structure in the ${ }^{13} \mathrm{C} /{ }^{12} \mathrm{C}$ ratio results from the overall $A^{1 / 3}$ scaling of the nuclear size. The amount of sensitivity to different forms for the ${ }^{13} \mathrm{C}-{ }^{12} \mathrm{C}$ nuclear structure difference is indicated by the small differences among the two curves and the data.

The new elastic scattering daia for $500 \mathrm{MeV} \vec{p}+{ }^{12,13} \mathrm{C}$ are described qualltatively with the microscopic RIA - Dirac equation model; quantitative fits are obtained with empirical potentials. The measured spin-flip probability does not agree in detail with any of the theoretical predictions shown here or in Ref. 2. However, the $D_{N N}$ data are far from conclusive and more measurements of this quantity are needed, as are continued improvements in the theoretical model. Finally, the ${ }^{13} \mathrm{C} /{ }^{12} \mathrm{C}$ differential cross section ratio is fit reasonably well with the relativistic DWBA model; this ratio is sensitive to the ${ }^{13} \mathrm{C}-{ }^{12} \mathrm{C}$ structure difference and can 
be used to constrain the nuclear structure model used in analyses of $\vec{p}+{ }^{13} \vec{C}$ elastic scattering data.

\section{References}

[1] G. W. Hoffmann et al., Phys. Rev. C 41, 1651 (1990).

[2] L. Ray, G. W. Hoffmann, M. L. Barlett, J. D. Lumpe, B. C. Clark, S. Hama, and R. L. Mercer, Phys. Rev. C37 1169 (1988).

\section{Figure Captions}

Figure 1. Elastic differential cross section, analyzing power, and spin-rotation data, and results of calculations, for $500 \mathrm{MeV} \vec{p}+{ }^{12} \mathrm{C}$. The solid and dashed curves are Dirac optical model fits and RIA - Dirac equation model predictions. respectively.

Figure 2. Elastic differential cross section, analyzing power, and spin-rotation data, and results of calculations, for $500 \mathrm{MeV} \vec{p}+{ }^{13} \mathrm{C}$. The solid and dashed curves are relativistic DWBA results based on Dirac phenomenology and the RIA optical potentials for ${ }^{12} \mathrm{C}$, respectively, that were used to compute the twelve nucleon core scattering amplitıde and relativistic distorted waves.

Figure 3. Data and RIA results for the spin-depolarization observable $D_{N N}$ for $500 \mathrm{MeV} \vec{p}+{ }^{13} \mathrm{C}$ elastic scattering. The solid and dashed curves are the relativistic DWBA results when the pseudovector and pseudoscalar forms for the Lorentz invariant $N N$ amplitude ${ }^{1}$ are used, respectively. The curves in the upper (lower) half of the figure correspond to use of the RIA (Dirac phenomenological) ${ }^{12} \mathrm{C}$ optical potential to compute the core scattering amplitudes and distorted waves.

Figure 4. Ratio of ${ }^{13} \mathrm{C}$ to ${ }^{12} \mathrm{C}$ elastic differential cross sections as a function of momentum transfer. The errors in the data include statistical uncertainties only. The solid and dashed curves are relativistic DWBA and Dirac optical model results, respectively. 
Figure 1
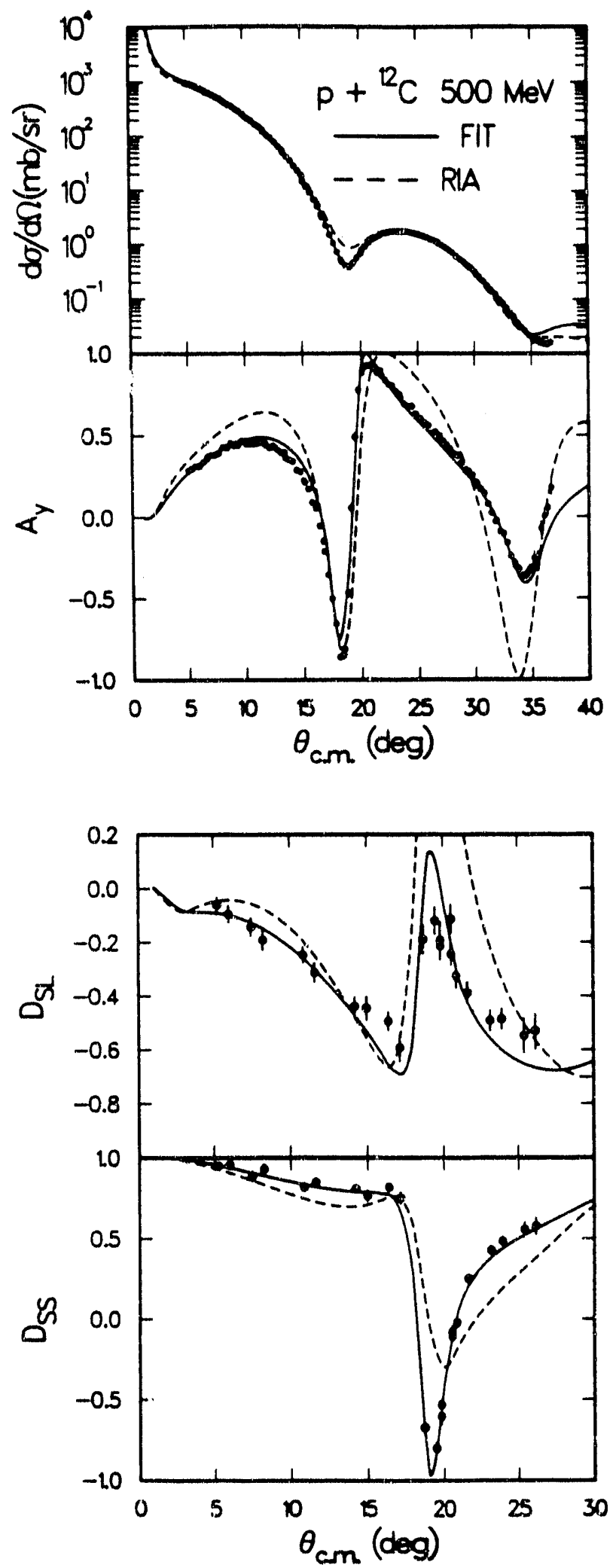
Figure 2

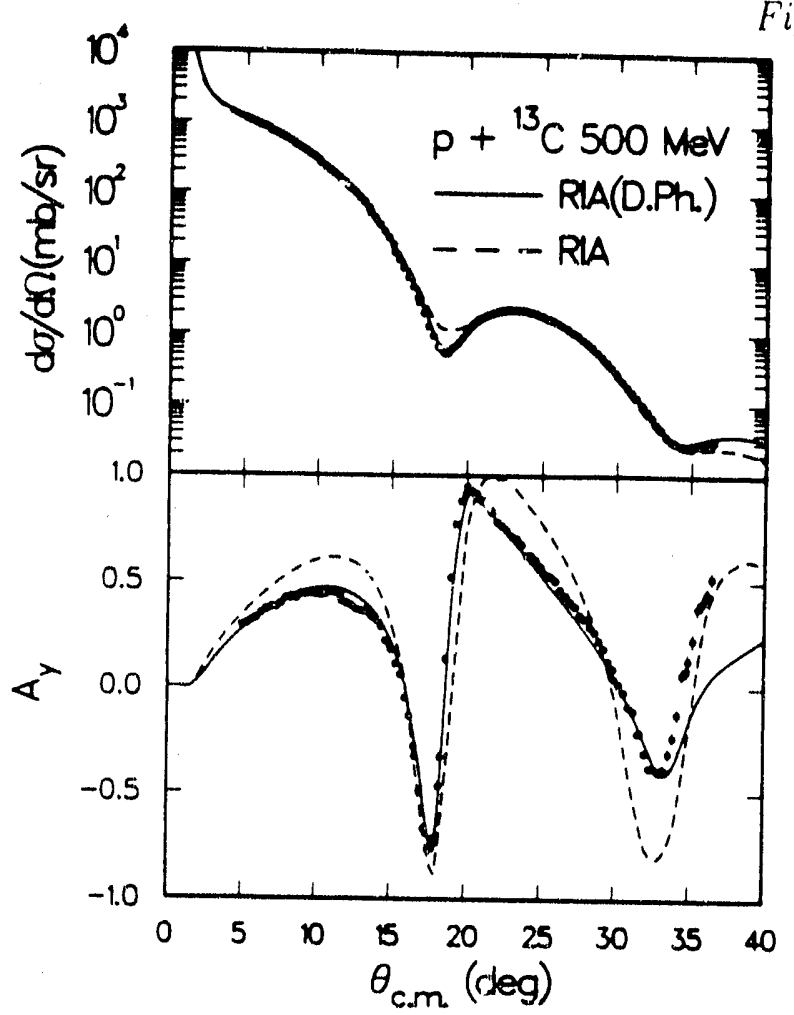

Figure 3

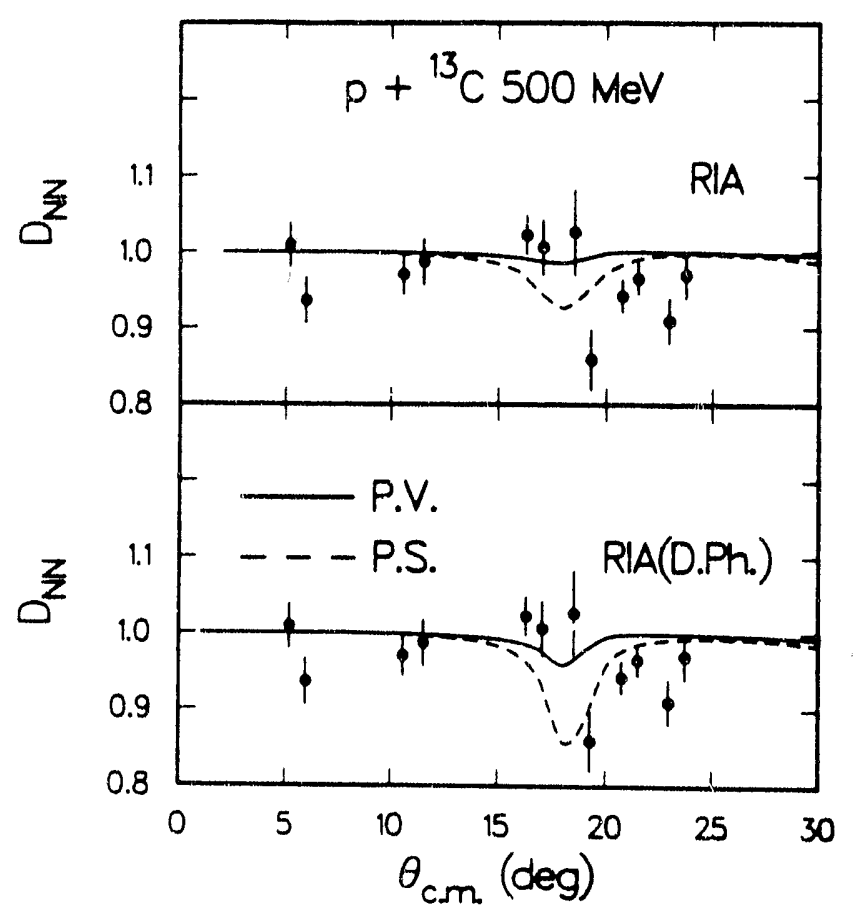

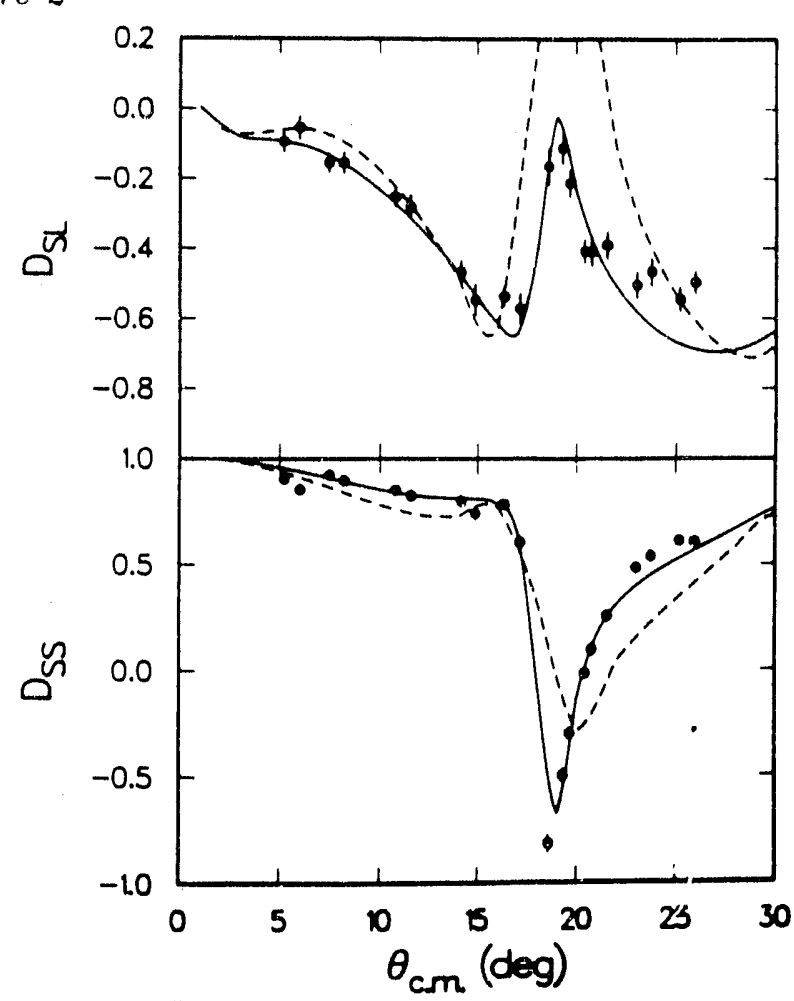

Figure 4

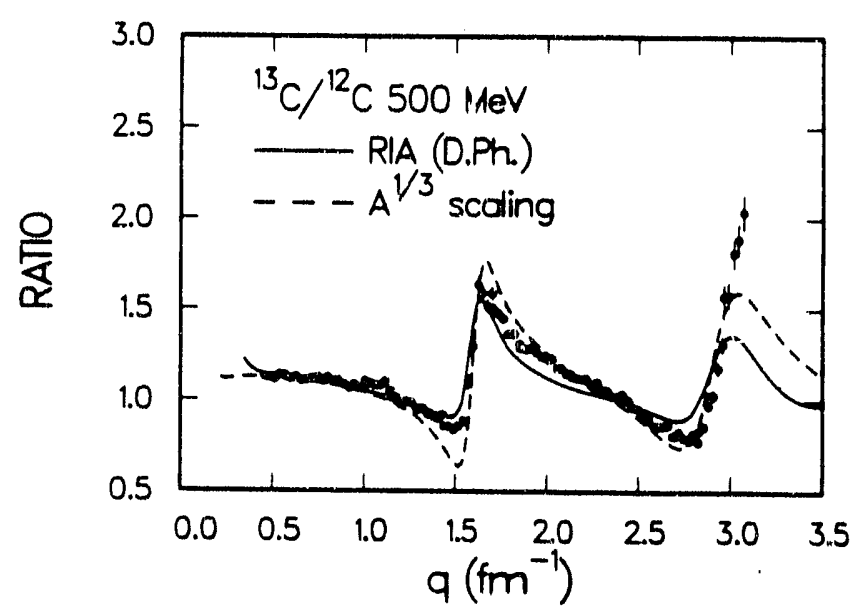




\subsubsection{EXP 955: Polarized Proton Elastic Scattering from Polarized ${ }^{13} \mathrm{C}$}

(G. W. Hoffmann, M. L. Barlett, W. Kielhorn, G. Pauletta, M. Purcell,

L. Ráy; LANL; NMSU; Minnesota; ASU; OSU; IBM;

G. W. Hoffmann, R. L. Ray, M. L. Barlett, J. Jarmer, spokesmen)

The medium energy proton-nucleus scattering data taken over the last 20 years for even-even target nuclei have stimulated calculations of density-dependent nucleonnucleon $(\mathrm{NN})$ effective interactions from a few $\mathrm{MeV}$ to $\sim 1 \mathrm{GeV}$, investigations of off-shell and fill-folding effects in the proton-nucleus optical poiential, studies of ground state matter densities and matter transition densities for collective excitations, and development of the relativistic impulse approximation (RIA), based on the Dirac equation.

However, studies of elastic and inelastic (collective) scattering for $\mathrm{J}^{\pi}=0^{+}$targets are sensitive mainly to just the no-spin and no-isospin transfer components of the $\mathrm{NN}$ interaction and the isoscalar, one-body densities of the target. Other types of reactions can be studied to investigate the spin and/or isospin transfer components of the NN interaction and the single particle aspects of nuclear structure. These reactions include non-natural-parity inelastic excitations and charge-exchange processes. Another possibility is proton elastic scattering from polarized, odd-mass nuclear targets. We performed the first such experiment at medium energies: a measurement of the target analyzing power $A_{\text {ooon }}$ (Ref. 1) and the spin-correlation pärameter $A_{\text {oonn }}$ (Ref. 1) for $\vec{p}+{ }^{13} \overrightarrow{\mathrm{C}}$ at $497.5 \mathrm{MeV}{ }^{2}$

The data were taken at LAMPF with the high resolution spectrometer (HRS). The target nuclei, ${ }^{13} \mathrm{C}$ and ${ }^{1} \mathrm{H}$, were polarized by using the dynamic nuclear polarization (DNP) technique. A single coil provided both the ${ }^{13} \mathrm{C}$ and ${ }^{1} \mathrm{H}$ nuclear magnetic resonance (NMR) signals, which were used to determine the ${ }^{13} \mathrm{C}$ and ${ }^{1} \mathrm{H}$ polarizations for the entire target material. The hydrogen polarization of the target was also measured by using a target polarimeter which monitored $\vec{p}+\vec{p}$ elastic scattering at $\theta_{\text {c.m. }}=46^{\circ}$, and relied upon the known values of $A_{y}=A_{\text {ooon }}$ and $A_{\text {oonn }}$ for $500 \mathrm{MeV} \vec{p}+\vec{p}$. The HRS was used to momentum analyze the scattered protons and generate missing mass spectra.

Data were taken between laboratory angles $12^{\circ}-30^{\circ}$ in about $1.5^{\circ}-2.0^{\circ}$ steps for the ${ }^{13} \mathrm{C}$ target and also for an empty target cell (to enable background corrections) and a thin, unpoiarized ${ }^{13} \mathrm{C}_{2} \mathrm{H}_{6} \mathrm{O}_{2}$ target (the ethylene glycol was sandwiched between two thin Be foils separated by $1 \mathrm{~mm}$ ) to allow accurate determination of the ${ }^{13} \mathrm{C} /{ }^{16} \mathrm{O}$ cross section ratios. The data from the dummy target and the thin ${ }^{13} \mathrm{C}$ target were crucial to the experiment because the ${ }^{13} \mathrm{C}$ and ${ }^{16} \mathrm{O}$ elastic peaks were not well-resolved except at the larger angles.

Small differences in the magnitudes of the beam polarization for normal and reverse directions and differences in the target polarization magnitudes for target "up" and target "down" runs were accounted for during data reduction.

The results for $A_{\text {ooon }}$ and $A_{\text {oonn }}$ are shown in Fig. 1 along with theoretical 
predictions. The errors shown account for all sources of statistical uncertainty; the measured yields dominated. Systematic errors in the beam and target polarizations are estimated to be small. Both data sets are smooth with respect to their angular distributions and display structure in the angular regions near the minima in the differential cross section $\left(18.5^{\circ}\right.$ and $34.5^{\circ}$ in the COM) as expected.

The theoretical results for $A_{\text {ovon }}$ and $A_{\text {oonn }}$ shown in Fig. 1 were computed in the disitorted wave Born approximation (DWBA) for both the RIA rnodel and the nonrelativistic impulse approximation (NRIA) model. For both calculations the ${ }^{13} \mathrm{C}$ wave function was assumed to be a pure $1 p_{\frac{1}{2}}$ neutron single particle state coupled to an inert ${ }^{12} \mathrm{C}$ "core," and the scattering amplitude, corresponding to the twelve-nucleon core, was obtained from a Dirac phenomenological optical model fit to the EXP $955 \mathrm{U} 500 \mathrm{MeV} \vec{p}+{ }^{12} \mathrm{C}$ elastic scattering data. The $\mathrm{NN} t$ matrices (in both the RIA and NRIA forms) in the proton-nucleus Breit frame were generated from the SP82 NN phase shift solution.

For the RIA-DWBA calculations the NN Lorentz invariant amplitudes were assumed to be given by the local McNeil-Ray-Wallace form. The valence neutron wave function was taken to be the $1 p_{\frac{1}{2}}$ eigenstate of the relativistic mean field (RMF) scalar and time-like vector binding potentials for ${ }^{12} \mathrm{C}$. The relativistic distorted waves were obtained through solution of the Dirac equation for $\vec{p}+{ }^{12} \mathrm{C}$ elastic scattering by using the Dirac phenomenological optical potential. The results are indicated in the figure by the solid curves. RIA-DWBA calculations were also done in which the relativistic enhancement of the lower component of the $1 p_{\frac{1}{2}}$ valence neutron wave function was suppressed, thus reducing its strength to the nonrelativistic limit. These results are shown by the dashed curves. Other calculations were made with a pseudovector, rather than pseudoscalar, form for the $\mathrm{NN}$ invariant amplitude, and with the twelve-nucleon core contribution to the isoscalar, three-vector current included. Both of these results lie between the solid and dashed curves in the figure.

For the NRIA - DWBA calculations the full NN $t$ matrix in the proton-nucleus Breit frame was used. Direct and exchange contributions were included, and the amplitudes were taken to be functions of momentum transfer only. The NR distorted waves were generated by solving the Schrödinger equation with relativistic kinematics by using the Schrödinger equivalent potential of the Dirac phenomenological optical potential for $\vec{p}+{ }^{12} \mathrm{C}$. The valence neutron wave function was assumed to be the (properly normalized) upper component of the RMF $1 p_{\frac{1}{2}}$ eigenstate. The NRIA-DWBA predictions are shown by the dashed-dotted curves in the figure.

Each of the calculations qualitatively describes the data for both observables, although all are lacking in necessary structure in the region from $15^{\circ}-25^{\circ} \mathrm{c} . \mathrm{m}$. Compared to the discrepancies between theory and data, the differences between the two RIA - DWBA predictions are small as are the differences between the RIAand NRIA - DWBA predictions.

The results reported here for $\vec{p}+{ }^{13} \overrightarrow{\mathrm{C}}$ at $500 \mathrm{MeV}$ show that such experiments are difficult, but possible, and that quality data can be obtained. These new types of 
scattering data are qualitatively explained by current theoretical models, but further improvements will evidently be necessary before quantitative understanding can be achieved.

\section{References}

[1] The notition for both the $N N$ and $p+{ }^{13} \mathrm{C}$ spin observables is that of $\mathrm{J}$. Bystricky, F. Lehar, and P. Winternitz, J. Phys. (Paris) 39, 1 (1978).

[2] G. W. Hoffmann et al., Phys. Rev. L'tt. (1990) (submitted).

\section{Figure Caption}

Figure 1. Experimental data and theor etical predictions for $\vec{p}+{ }^{13} \overrightarrow{\mathrm{C}}$ target spin observables $A_{\text {ooon }}$ (upper portion) and $A_{\text {oonn }}$ (lower portion) at $497.5 \mathrm{MeV}$. The RIA-DWBA predictions assuming the RMF (nonrelativistic) value for the valence neutron lower component wave function are indicated by the solid (dashed) curves. The NRIA-DWBA predictions are given by the dashed-dotted curves.

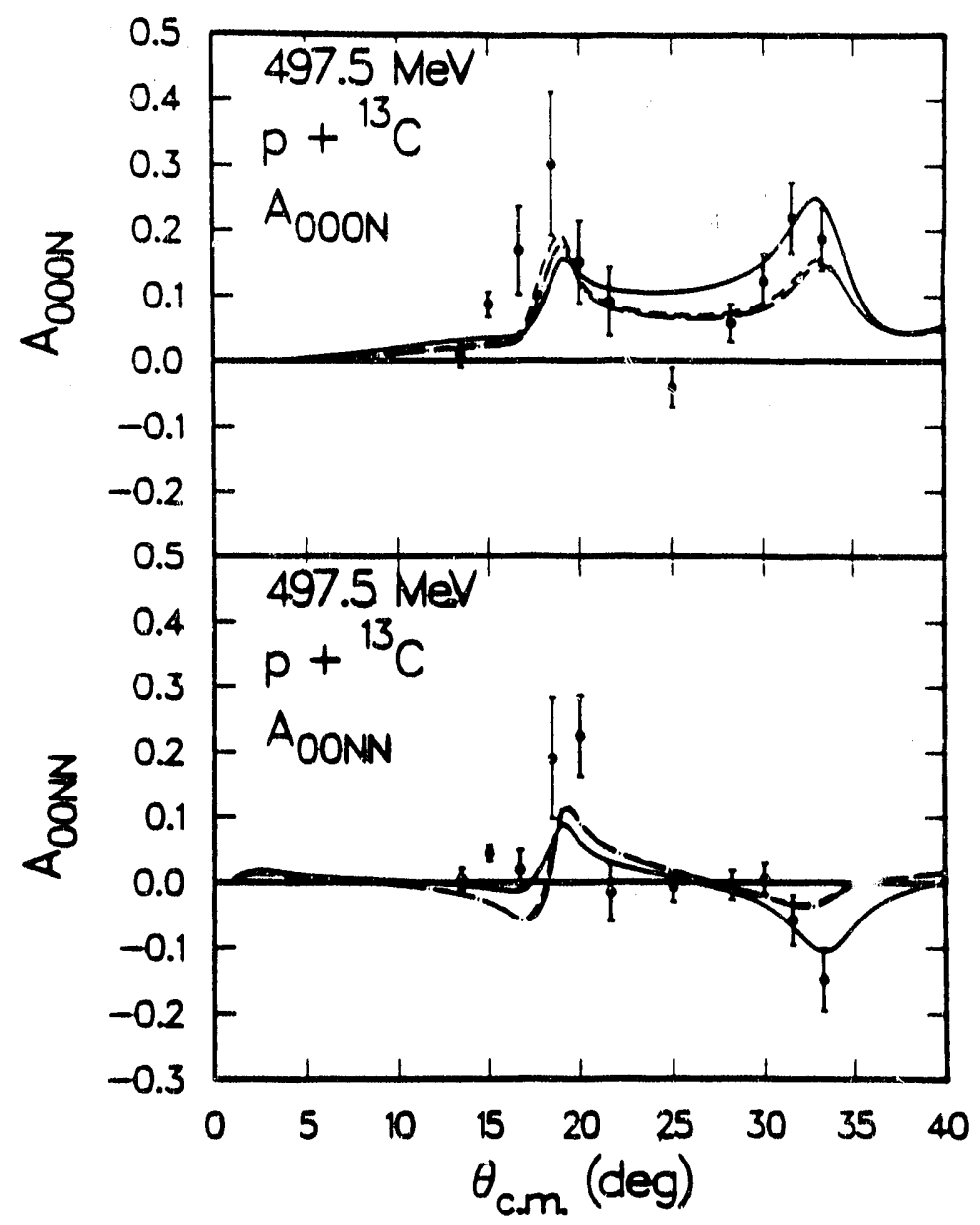




\subsubsection{EXP 1079: $p+$ A Precision Elastic Forward Angle Cross Sections}

(G. W. Hoffmann, M. L. Barlett, G. Pauletta, L. Ray, D. Read S. Worm, A. Smith, M. Purcell, Z. Mujtaba; LANL; Texas A\&M;

G. W. Hoffmann, N. Tanaka, J. F. Amann, spokesmen)

A comparison of observables predicted by scattering models which use either relativistic (RIA) or nonrelativistic (NRIA) dynamics indicates ${ }^{1}$ significant differences in the magnitudes of the $500-800 \mathrm{MeV} p+{ }^{16} \mathrm{O},{ }^{40} \mathrm{Ca},{ }^{208} \mathrm{~Pb}$ differential cross sections in the forward-angle, Coulomb-nuclear interference region (see Fig. 1). At $500 \mathrm{MeV}$ somie of the differences are $15-25 \%$. This result is stable with regard to uncertainties in the theoretical calculations $( \pm 2-6 \%)$ including medium effects, and suggests that techniques be developed to perform the required experiment to test the various models. It has also been pointed out that precise cross section data in the Coulomb-nuclear region would set important constraints on relativistic models of the virtual pair process and the scalar densities of the target nucleus. Once developed, the techniques could also be used to renormalize to the level of $1 \%$ the vast quantity of $p+A$ elastic scattering data that has been taken at LAMPF over the past 15 years. Such accuracy is particularly useful for nuclear structure studies.

The first phase of EXP 1079 was the development of the techniques required for a $1 \%$ experiment: Of particular importance are accurate integrated beam flux determination $(0.5 \%)$, precise polar scattering angle determination $\left(0.001^{\circ}\right.$, and accurate solid angle determination $(0.1 \%)$.

We have had two development runs in which we have commissioned the new HRS Faraday cup, a high gain (xenon-ethane $600 \mathrm{~mm} \mathrm{Hg}$ ) ion chamber, the EXP 1072 beam counting scintillator telescope as modified for this experiment, and the new Line $\mathrm{C}$ inductive pickup coil (LCCM). We have been able to show that with care there is sufficient overlap in the beam intensity regions over which these devices operate that together they can redundantly determine beam current over the range from $10^{4} / \mathrm{s}$ to several nA with better than $1 \%$ absolute accuracy.

We have demonstrated that the solid angle can be determined to the $0.1 \%$ level using brass slits of aperture $10 \%$ of the HRS acceptance. Slit edge scattering was demonstrated to have negligible effect on the solid angle defined by the aperture (note that medium energy protons that go through the slit material loose about $10 \mathrm{MeV}$ so that they are shifted in the missing-mass spectrum generated by the HRS; nominal HRS resolution is $100 \mathrm{ke}$ ).

Small wire chambers, for use in the HRS scattering chamber, have been designed and are under construction. They will be used for tuning the zero-phasespace pencil beam and determining the spatial coordinates of the beam optic axis to about 50 microns, allowing a very precise determination of the absolute polar scattering angle.

We have developed the tools required to perform production runs, and will ask 
for production time at the January, 1991 PAC meeting.

\section{References}

[1] L. Ray, G. W. Hoffmann, M. L. Barlett, and N. Ottenstein, Phys. Rev. C 37, $224(198 ;)$.

\section{Figure Caption}

Figure 1. Uncertainty bands for the forward angle differential cross sections for $500 \mathrm{MeV} p+{ }^{40} \mathrm{Ca}$ and ${ }^{208} \mathrm{~Pb}$ from the standard NRIA (shaded bands between dashed curves) and RIA (shaded bands between the solid curves), from Ref. 1.
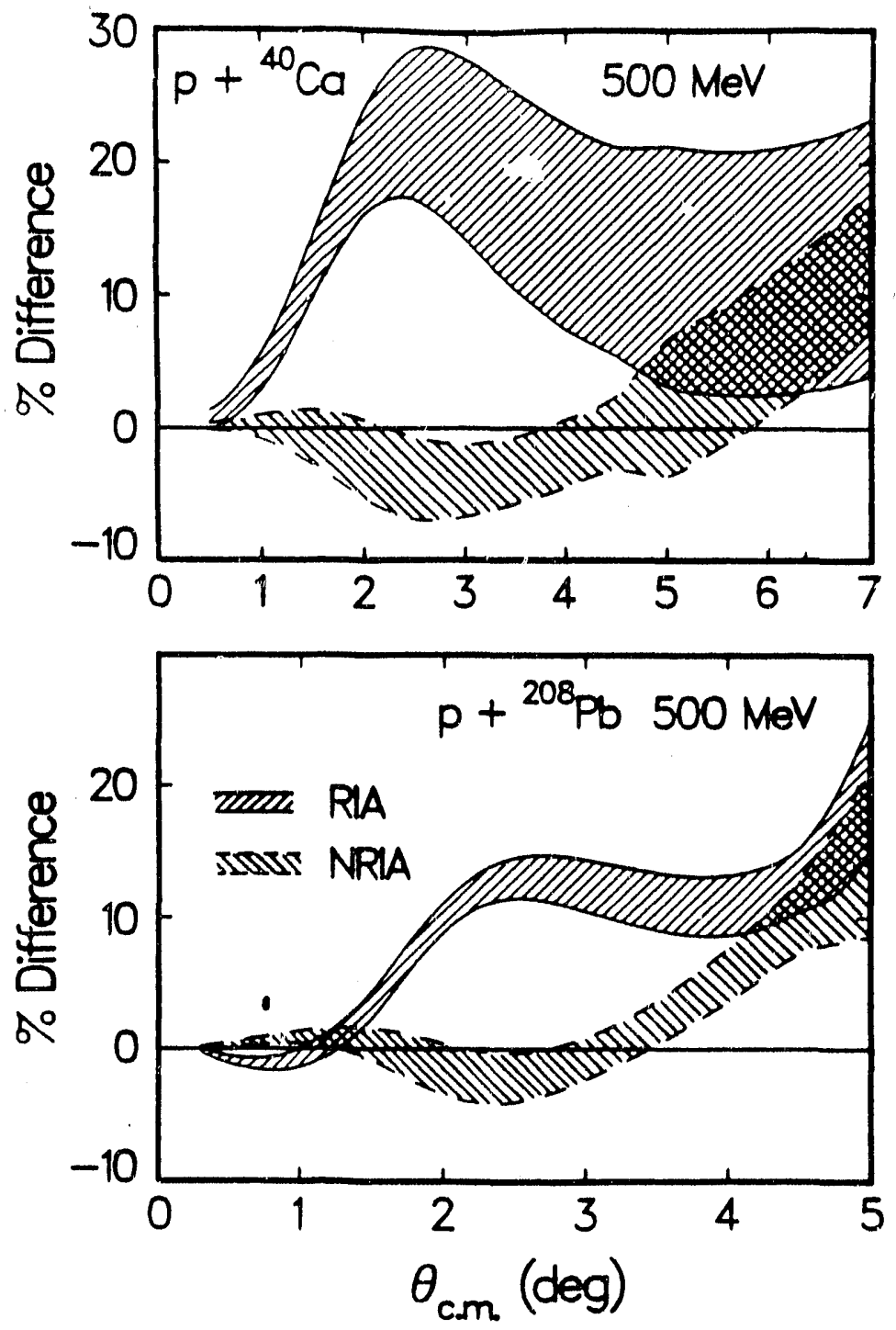


\subsubsection{EXP 1119: $p+d$ Elastic Absolute Cross Sections}

(M. L. Barlett; LANL; UCLA; Rice; Rutgers; Texas A\&M;

M. McNaughton, spokesman)

Since the $p+d$ system is one of the simplest many-body systems, the study of $p+d$ elastic scattering at intermediate energies may be a possible way to test some cundamental aspects of hadron - nucleus scattering mudels. For example, the data may be useful in determining the role played by 3-body forces in the uuclear medium. A complete set of scattering observables is desireable for the most rigorous tests. Among the possible observables that can be measured, the unpolarized differential cross sections play an essential role in determining the overall scale of the amplitudes, because bilinear combinations of the scattering amplitudes are expressed in terms of spin-observables times the unpolarized differential cross sections. Thus, the accuracy of the differential cross section data directly affects the interpretation of the spin-observa'ie data. Particularly desireable, is quality data near $800 \mathrm{MeV}$, an energy where considerable $p+d$ spin-observable data exist ${ }^{1}$ and an energy where some of the available cross section data ${ }^{2}$ have been questioned.

The experiment, ${ }^{3}$ performed at LAMPF, took advantage of the apparatus developed for EXP 1072, the $p+p$ absolute differential cross section experiment. A liquid deuterium target was used, and data were taken at beam energies (center of target) of $641.3 \mathrm{MeV}$ and $792.7 \mathrm{MeV}$. As in EXP 1072 both scattered and recoil particles were detected with a redundant detection scheme that allowed precise efficiencies to be determined. The integrated beam flux was also determined as in EXP 1072 using a 3-counter scintillator telescope in the beam upstream of the target to directly count beam particles. An elaborate electronic timing scheme plus the use of Poisson statistics made it possible to correct for more than one beam particle in any given $\mathrm{rf}$ micropulse.

The new data ${ }^{3}$ are shown in Fig. 1, together with the $800 \mathrm{MeV}$ data of Winkelman ${ }^{2}$. Typical uncertainties in these new data are 2-3\% (absolute). Using these new data we have been able to resolve the controversy concerning the older data set $^{2}$. The problem seems to be that the inverse of the correct Jacobian was used in transforming the data between the laboratory and the center-of-momentum coordinate system. The new data obained in EXP 1119 should be useful for the continued theoretical study of the intermediate energy $p+d$ elastic scattering process.

\section{References}

[1] G. J. Igo, in the Proceedings of the Int. Symp. on Medium Energy Physics, June, 1987, Beijing, China.

[2] E. Winkelmann et al., Phys. Rev. C 21, 2335 (1980). 
[3] E. Gülmez et al., Phys. Rev. C (1990) (submitted).

Figure Caption

Figure 1. Center-of-momentum cross sections as a function of center-of-momentum scattering angle for 641.3 and $792.7 \mathrm{MeV} p+d$ elastic scattering.

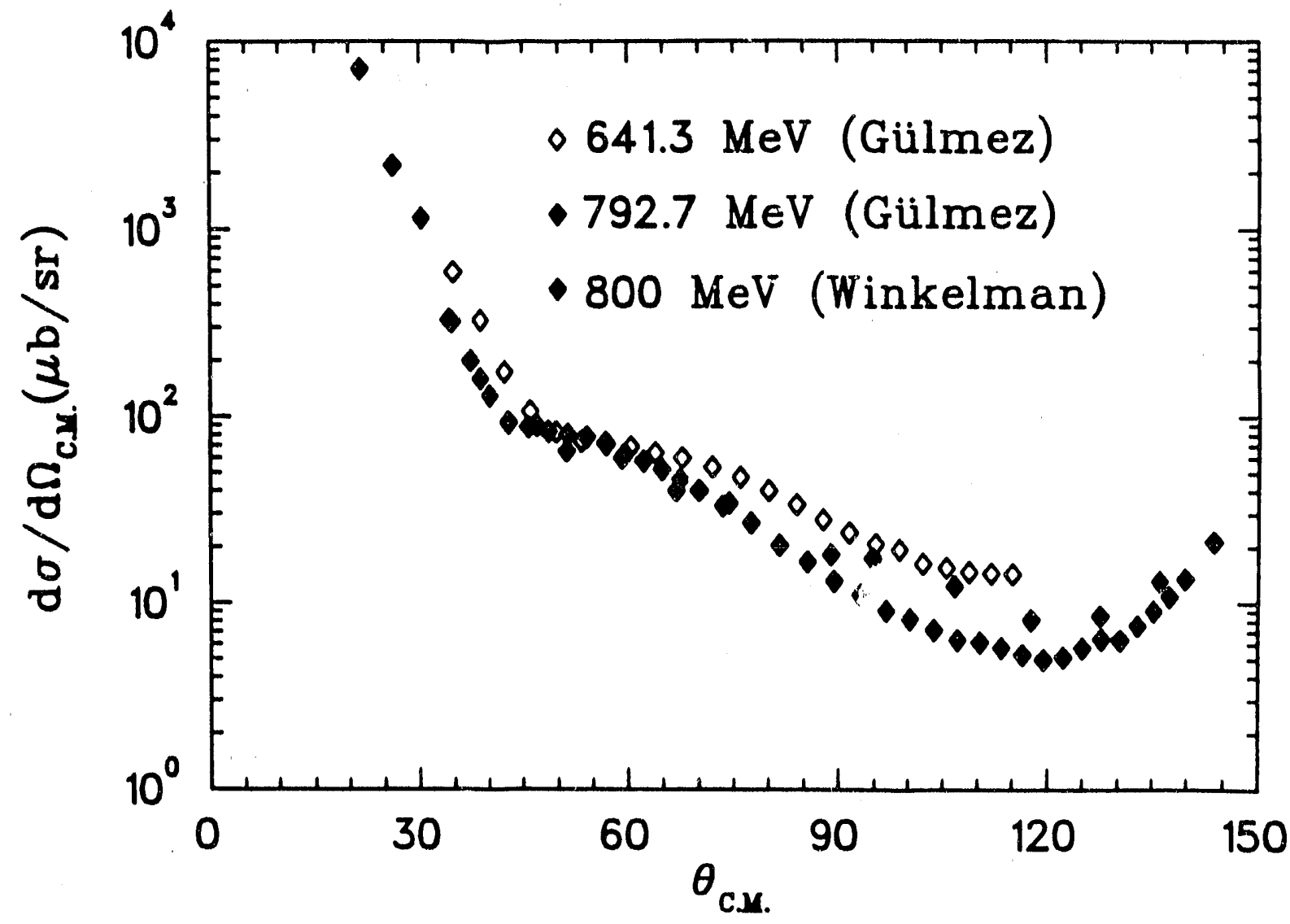




\subsection{Proton Induced Inelastic Scattering and Reactions}

\subsubsection{EXP 626: Polarization Transfer for Inclusive Proton Nucleus Inelastic Scattering at $800 \mathrm{MeV}$}

(M. Barlett, G. Hoffmann, J. Marshall; Rutgers; Institute for Atomic

Energy, Beijing, China; LANL; C. Glashausser, J. McGill, spokesmen)

The high-excitation energy response of the nucleus to mediurn energy probes has been the subject of considerable interest. A fundamental question is whether the nuclear response in this region is close to that of a noninteracting gas of fermions or whether there are specific many-body effects that are important even at highexcitation energies. If a Fermi gas picture is valid and distortions and Fermimotion effects are small, then spin-observables such as the analyzing power $A_{y}$ or the polarization-transfer coefficients $D_{i j}$ for inelastic proton scattering should be very close to the values for nucleon-nucleon $(N N)$ scattering. Deviations from the Fermi gas predictions could result, for example, from nuclear structure effects like dynamical correlations which persist even at high-excitation energy. Another medium effect is suggested by applications of the Dirac equation to the description of nuclear structure and reactions. Calculations indicate that many of these effects are most easily seen in the behavior of spin observables in scattering experiments.

We have measured ${ }^{1}$ all of the parity allowed polarization-transfer coefficients $\left(D_{N N}, D_{S S}, D_{L L}, D_{L S}, D_{S L}\right)$ for inclusive $800 \mathrm{MeV}$ proton scattering from ${ }^{1} \mathrm{H},{ }^{2} \mathrm{H}$, and ${ }^{12} \mathrm{C}$. The data were taken at laboratory scattering angles of $5^{\circ}, 11^{\circ}$, and $20^{\circ}$, and cover the excitation energy range from the quasielastic region (elastic scattering from ${ }^{1} \mathrm{H}$ ) to the delta region. These data comprise a survey of spin observables in this region which complement the earlier cross section and analyzing power data taken in EXP $642 .{ }^{2}$

The data are shown in Figs. 1-5, where they are plotted as a function of energy loss $\omega$. The arrows marked " $N$ " in each figure indicate the energy loss corresponding to free $p+{ }^{1} \mathrm{H}$ scattering at each angle. The arrows marked $\pi$ indicate the energy loss corresponding to an excitation of $300 \mathrm{MeV}$ in the $p+{ }^{1} \mathrm{H}$ systemenough to excite the center of the first delta resonance. The curves on the ${ }^{1} \mathrm{H}$ plots are unitary model predictions. ${ }^{3}$ The solid curves on the ${ }^{12} \mathrm{C}$ data in the quasielastic and dip regions are nonrelativistic slab model calculations. ${ }^{4}$ Relativistic plane wave impulse approximation predictions are shown as the dashed curves. ${ }^{5}$

The data for each observable at each angle show great similarity for the three targets. There are no dramatic differences which would provide clear evidence of strong medium effects on any observable. The differences that do exist are seldom greater than aluut two standard deviations and they show few systematic trends.

The theoretical curves for ${ }^{12} \mathrm{C}$ in the quasielastic region are in qualitative agreement with the data; only small differences between the relativistic and nonrelativistic calculations are specifically due to relativity. The two sets of theoretical 
predictions contain different nuclear ingredients, yet the net effect of these differences is small. The remaining discrepancies that $a$ re apparent in the comparison of theory and experiment in a few regions are interesting and deserve further investigation.

\section{References}

[1] R. Fergerson et al., Phys. Rev. C 38, 2193 (1988).

[2] J. A. McGill et al., Phys. Rev. C 29, 204 (1984).

[3] J. Dubach, W. M. Kloet, and R. R. Silbar, Nucl. Phys. A466, 573 (1987).

i4] R. D. Smith (private comm niration)

[5] D. P. Murdock and C. J. Ho-switz, Phys. Rev. C 35, 1442 (1987).

\section{Figure Captions}

Figures 1-5. Measured values of the polarization transfer observables $D i j$ for inclusive proton scattering at $800 \mathrm{MeV}$ for the targets and scattering angles noted. The curves are from Refs. 3-5.

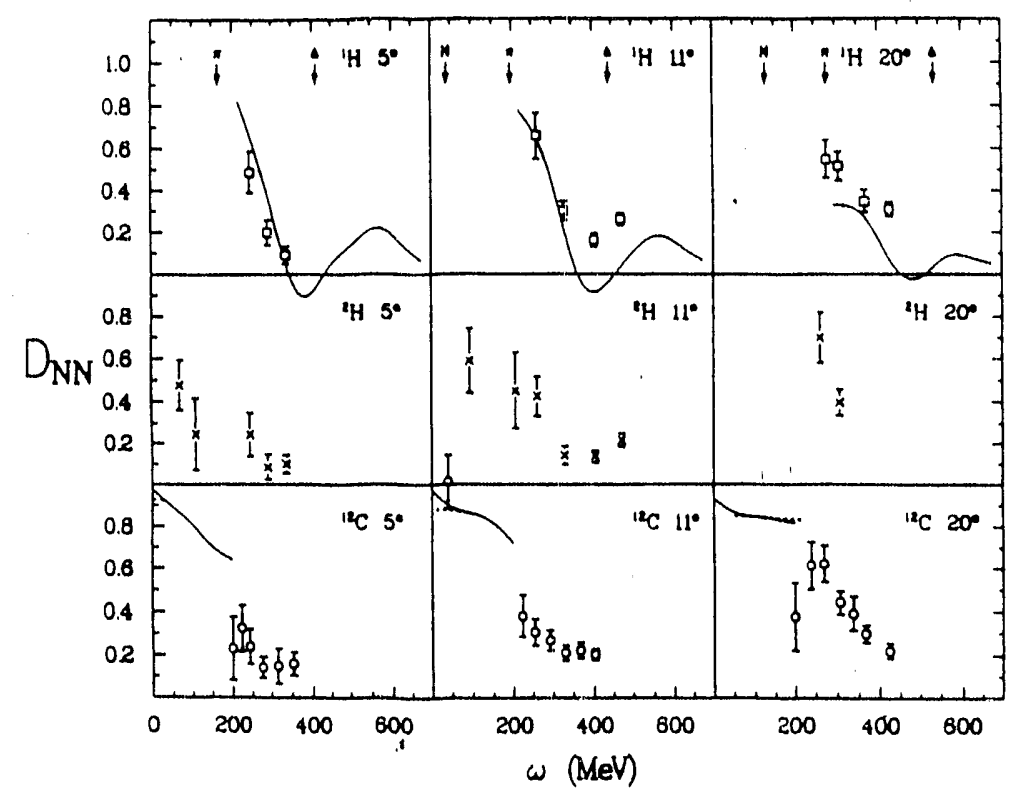

Figure 1 

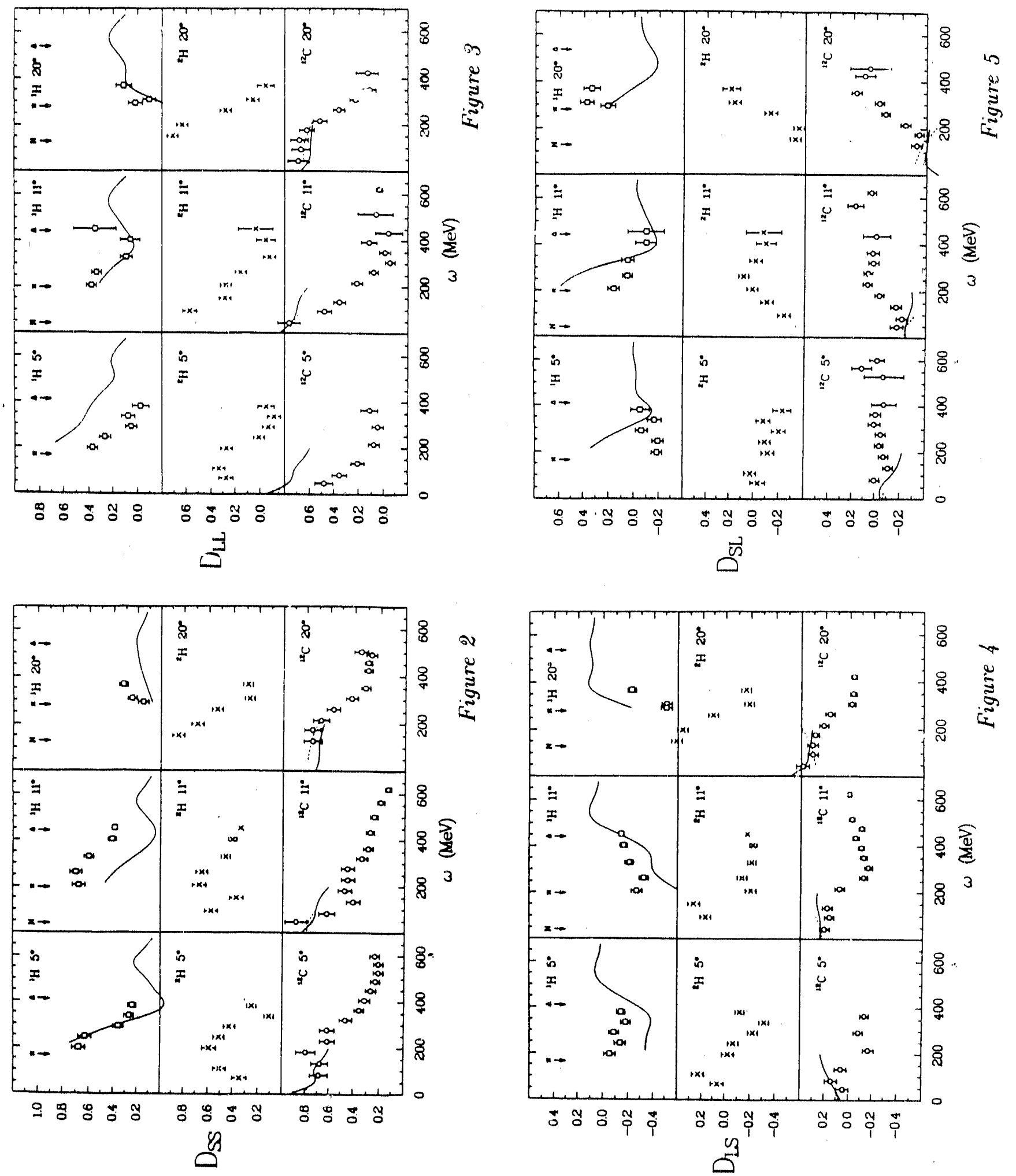


\subsubsection{EXP 973: Search for Narrow Resonances in the $B=2$ Missing Miass Spectrum from $p+{ }^{3} \mathrm{He}$ Reactions}

(M. L. Barlett, D. Ciskowski, G. W. Hoffmann, G. Pauletta, M. Purcell;

Udine; Minnesota; LANL; Virginia; M. Gazzaly, G. Pauletta, N. Tanaka, spokesmen)

Reports of narrow $\left(\Gamma_{1 / 2} \leq 50 \mathrm{MeV}\right)$ structure in the $\mathrm{B}=2$ missing mass spectra have attracted considerable interest in the past few years. ${ }^{1}$ Prominent among these are the $\mathrm{I}($ isospin $)=1$ structures seen in the ${ }^{3} \mathrm{He}(p, d) \mathrm{X}$ reaction. Excitation functions for this reaction, measured at Saclay, have shown evidence of narrow structures at several energies and the credibility of these measurements is enhanced by agreement between these energies and resonance energies predicted by a rotation model based on a virtual $\mathrm{NN} \pi$ bound state. ${ }^{2}$ Other structures, reported to occur in a variety of reactions, appear to be well accomodated by this model.

The purpose of EXP 973 was to corroborate the Saclay results, by measuring both the differential cross section and analyzing power using the HRS at LAMPF. These were measured at a deuteron scattering angle of $22^{\circ}$ (lab) at an incident proton momentum of $1.46 \mathrm{GeV} / \mathrm{c}$. The data from this experiment are shown in Figs. 1-2 (from Ref. 1). In Fig. 1 differential cross section measurements from this experiment (solid dots) are compared with data from Saclay taken at $1.4 \mathrm{GeV} / \mathrm{c}$ (crosses). In Fig. 2 analyzing power data obtained in this experiment are shown both before (upper part) and after (lower part) dummy target background subtractions were made. The curves through the data are fits using sums of gaussians. The vertical lines in both figures correspond to predicted resonance energies. ${ }^{2}$ The vertical arrows correspond to the cnergies at which possible structures were observed at Saclay. The possibility of structure is clearer in the missing mass spectra of the analyzing powor. All but one of the maxima in the data correspond to resonance energies predicted in our range of missing mass. Interpretation of the data is not unambiguous due to the narrow range covered in missing mass and because of the lack of theoretical predic , ns for the benavior of the analyzing power near the resonances. These results do, however, provide ample incentive for more measurements with better statistics $c$.er a wider range of missing mass. Further experimental runs to accomplish this are planned.

\section{References}

[1] L. Santi et al., Phys. Rev. C 38, 2466 (1988).

[2] M. H. MacGregor, Phys. Rev. Lett. 42, 1724 (1979). 


\section{Figure Captions}

Figure 1. Differential cross sections for the reaction ${ }^{3} \mathrm{He}(\vec{p}, d) \mathrm{X}$ from this experiment (solid dots) are compared with Saclay data (crosses).

Figure 2. Analyzing power data from this experiment before (upper part) and after (lower part) subtracting dummy target backgrounds.

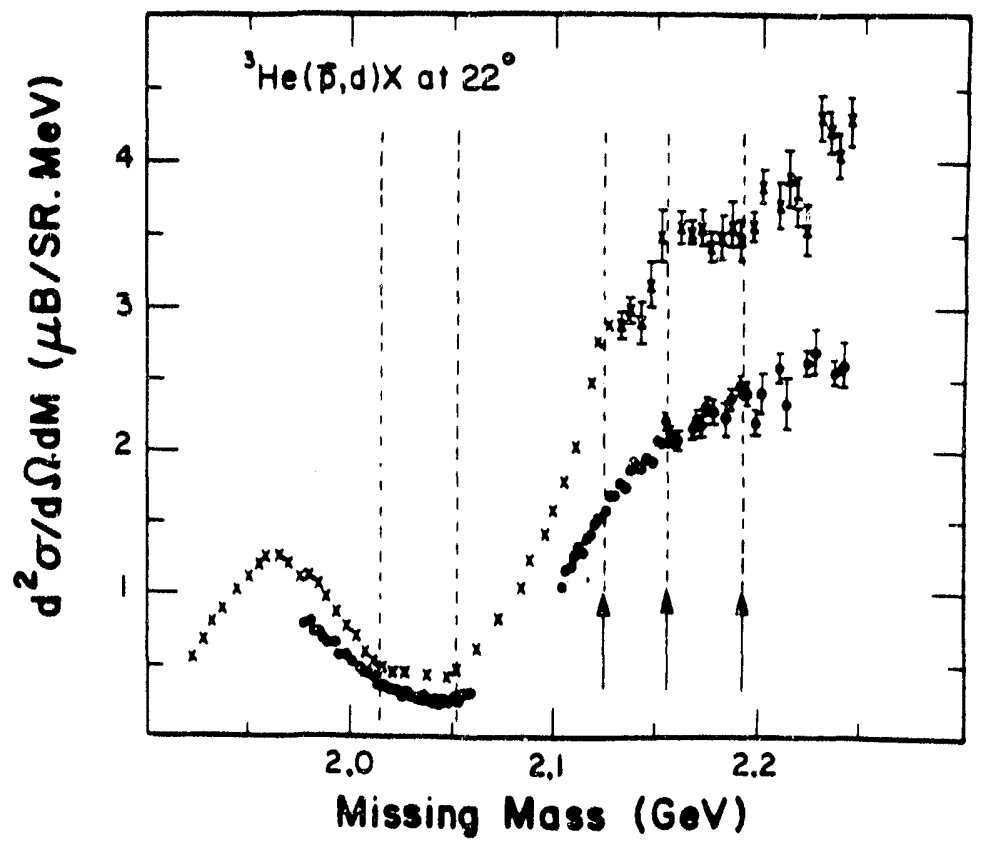

Figure 1

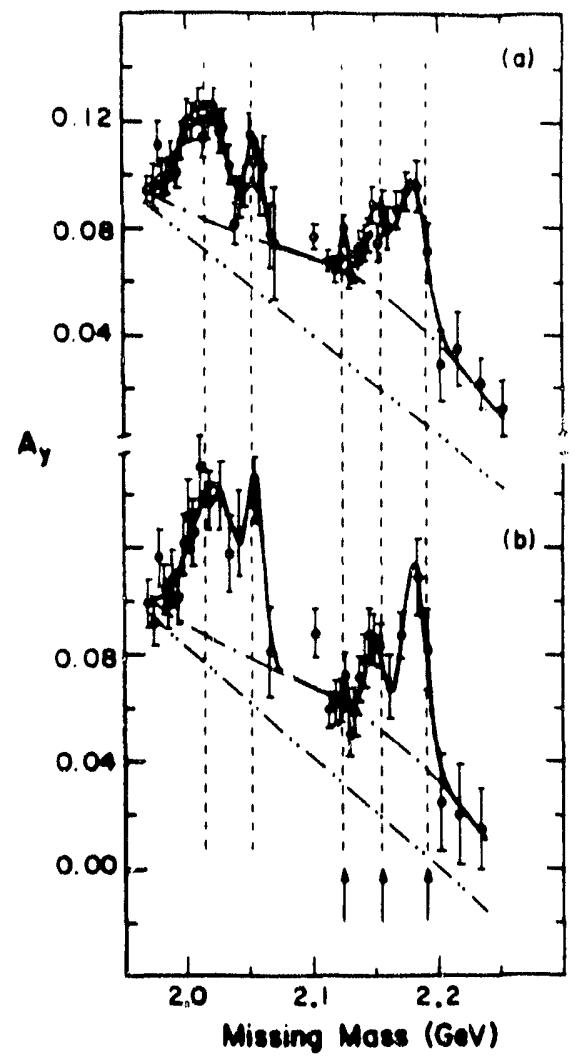

Figure 2 


\subsubsection{EXP 1131: Measurement of Polarization Transfer for $800 \mathrm{MeV}$ Inclusive Proton Scattering at the MRS}

(G. Hoffmann, D. Read, S. Worm, A. Smith, Z. Mujtaba; Rutgers; LANL; Georgia; Orsay; C. Glashausser, G. W. Hoffmann, K. Jones, spokesmen)

See the discussion concerning EXP 626 for the motivation for $\left(p, p^{\prime}\right)$ experiments to probe the high excitation energy response of the nucleus at medium energies. EXP 626 was done with the high resolution spectrometer (HRS), and because of time limitations and intrinsic constraints from spin precession in the HRS dipoles, the data have relatively large statistical errors and have a number of gaps for various observables at different outgoing proton energies. These data do noi have the statistical accuracy to distinguish small but possibly significant effects of the nuclear medium on various spin observables, and the gaps and poor statistics in the data for some observables in some energy-loss regions make it difficult to discuss trends. The fact that each data point represents a particular magnetic field setting also might contribute to small systematic errors from one setting to the next.

The design of the medium energy resolution spectrometer (MRS) at LAMPF (Fig. 1) suggests that this instrument is ideal for nuclear response measurements in the continuum. We have initiated an experimental program using the MRS, whose purpose is to both commission and test the spectrometer over a wide energy range, and yield new physics through a measurement of a complete set of polarization transfer data for ${ }^{1} \mathrm{H},{ }^{2} \mathrm{H},{ }^{12} \mathrm{C}$, and ${ }^{40} \mathrm{Ca}$ at $20^{\circ}$ with energy losses extending to about $500 \mathrm{MeV}$. The entire energy loss region can be spanned using $4 \mathrm{MRS}$ magnetic field settings, and at $20^{\circ}$ the quasielastic peak is particularly well-defined.

The initial shakedown run for this experiment has recently been completed. A considerably more than anticipated amount of time was devoted to installing and commissioning the newly constructed MRS focal plane polarimeter (FPP) and understanding the optics of the beam line as well as those of the MRS itself. Although in principle the MRS-FPP is essentially the same as the HRS-FPP in theory of operation, operation of the MRS-FPP system is in many ways much more complex than it is for the HRS-FPP system owing to the large momentum acceptance of the MRs $(30 \%)$ compared to the HRS $(2 \%)$. Thus, although it was possible to understand and operate the MRS system over a restricted region of the focal plane, the task of understanding it over the full momentum acceptance $(30 \%)$ was a time-consuming and difficult task. Off-line analysis of the data taken during this run has now provided a reasonable calibration and understanding of the MRS-FPP system, and we are now in a position for production runs to begin. 


\section{Figure Caption}

Figure 1. Schematic drawing of the new rnedium resolution spectrometer (MRS) at LAMPF.

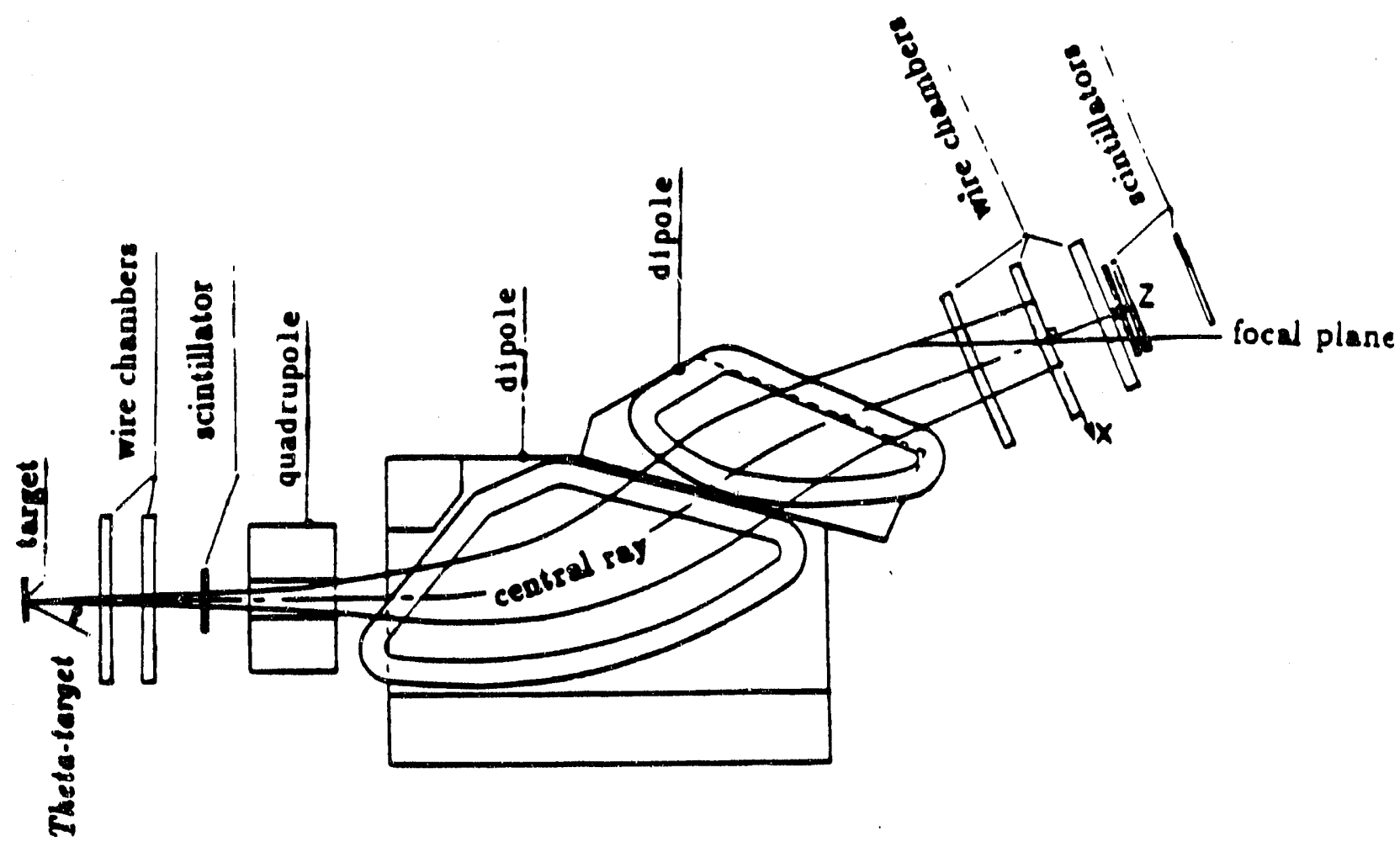




\subsubsection{EXPS 1052, 1061, 1062: NTOF Calibration and $(p, n)$ Charge Exchange Reactions at Medium Energies}
(D. Ciskowski, M. L. Barlett; LANL; OSU; Indiana; Ohio; Colorado;
UCLA; T. A. Carey, J. B. McClelland, J. Shepard, E. Sugarbaker,
T. Taddeucci, spokesmen)

The newly commissioned neutron time-of-flight (NTOF) facility at LAMPF consists of a beam line, swinger magnets, and neutron detectors which provide the capability for studying $(p, n)$ charge exchange (CEX) reactions at intermediate energies. A variable length flight path up to $600-\mathrm{m}$ is available together with a neutron detection system which allows measurement of neutron polarization. Details of the neutron detection system may be found in Refs. 1-4.

The study of $(p, n)$ charge exchange at intermediate energies provides an opportunity for several new areas of investigation. Included among these are the following: (1) direct examination of the isovector components of the effective $N N$ interaction, both spin-flip and non-spin-flip parts, (2) determination of Fermi (F) and Gamow-Teller (GT) transition form factors for nuclear structure studies, (3) searches for Fermi and GT strength in the continuum region of excitation, (4) further study of relativistic versus nonrelativistic descriptions of nuclear reactions and issues associated with these relativistic approaches such as pseudoscalar (PS) or pseudovector (PV) forms for the Lorentz invariant NN interaction, (5) direct study of the modification of the elemental two-body process $p+n \rightarrow n+p$ in the nuclear medium via quasielastic $(p, n)$ measurements, $(6)$ study of isovector giant resonances, and (7) study of direct $\Delta(1232 \mathrm{MeV})$ production in nuclei. Experiments in some of these areas have begun.

The purpose of EXP 1052 was to calibrate the neutron detection system over the full range of neutron energies available from the LAMPF accelerator using a tagged neutron beam. The differential and integral efficiencies were determined as well as position resolution and other systematic effects. The tagging was accomplished with an auxiliary proton detection arm which detected protons from elastic $n+$ $p$ ecattering using the neutron beam in area $\mathrm{BR}$ and a $\mathrm{LH}_{2}$ target. Measured recoil proton angles and momenta determined the scattered neutron kinematics, thus allowing a calibration of the neutron detector array. Neutron energies from 50 to $750 \mathrm{MeV}$ were measured. Integral efficiencies for the entire detector are roughly energy-independent and are about 0.09 . Additional studies of differential efficiencies, cross talk systematics and other systematics have been completed.

EXP 1061 obtained the first set of CEX angular distribution data with NTOF for the reaction ${ }^{15} \mathrm{~N}(\vec{p}, n){ }^{15} \mathrm{O}$ (g.s.) at $494 \mathrm{MeV}$. Because of the good energy separation $(5.2 \mathrm{MeV})$ between ground and first excited states in ${ }^{15} \mathrm{O}$, the short flight path (82-m) which was initially available provided adequate resolution for the experiment. An overall resolution of $2.7 \mathrm{MeV}$ was obtained using the beam rebuncher. The target material consisted of ${ }^{15} \mathrm{~N}$ enriched melamine of thickness $705 \mathrm{mg} / \mathrm{cm}^{2}$. 
Differential cross section and analyzing power data were obtained and are shown in Fig. 1 in comparison with a variety of theoretical predictions. The solid and dashed curves represent RIA-DWBA predictions assuming the PV and PS forms for the Lorentz invariant interaction, ${ }^{5}$ respectively. Relativistic enhancement of the lower component of the valence nucleon wave function was assumed in both calculations. The dotted curves represent NRIA-DWBA predictions. ${ }^{6}$ No available calculations are able to reproduce the forward angle analyzing power data. Further theoretical study of this reaction will continue.

The goal of EXP 1062 was to provide data for pure Fermi and GT $(p, n)$ CEX transitions for the same target via the reaction ${ }^{14} \mathrm{C}(p, n)^{14} \mathrm{~N}\left(0^{+}, 2.31 \mathrm{MeV} ; 1^{+}\right.$, $3.95 \mathrm{MeV})$. This was done ${ }^{2}$ at $0^{\circ}$ at 494,644 and $795 \mathrm{MeV}$ using the full $617-\mathrm{m}$ flight path. The purpose was to study the isovector spin-flip $\left(J_{\sigma \tau}\right)$ and non-spinflip $\left(J_{\tau}\right)$ strengths of the $\mathrm{NN}$ effective interaction and the ratio of the two as functions of energy. Discrepancies between measured strengths and the free NN amplitude values at lower energies have led to speculation about strong (weak) density dependence in $J_{\tau}\left(J_{\sigma \tau}\right)$. The data are shown in Fig. 2 where the Fermi and GT $0^{\circ}$ cross sections are shown in the lower and middle portions of the figure, respectively. In the upper portion the ratio, $R^{2}$ (essentially $\left|J_{\sigma \tau} / J_{\tau}\right|^{2}$ ) is shown. The predictions based on the free NN amplitudes are shown by the dashed lines; those based on a G-matrix calculation ${ }^{7}$ are given by the solid curves. The apparent large suppression of the Fermi strength at the higher energies is not understood at present.

Other $(p, n)$ work engaged in by our group includes, neasurements from 80 $795 \mathrm{MeV}$ of ${ }^{7} \mathrm{Li}(p, n)^{7} \mathrm{Be}(\mathrm{g}$.s. $+0.43 \mathrm{MeV})(494,644$ and $795 \mathrm{MeV}$ at NTOF $){ }^{3}$ Differential cross section angular distributions and integrated total cross sections for this reaction were obtained. Such data are needed for normalizing other CEX measurements. Also $0^{\circ}$ Fermi and GT cross section measurements at 492 and $590 \mathrm{MeV}$ for ${ }^{7} \mathrm{Li},{ }^{11} \mathrm{~B}$, and ${ }^{12,13,14} \mathrm{C}$ were made at the WNR facility at LAMPF for the purpose of studying the $J_{\sigma \tau}$ and $J_{\tau}$ strengths. $^{4}$

\section{References}

[1] D. Ciskowski, Ph. D. thesis, The University of Texas at Austin (1988).

[2] E. Sugarbaker et al., Phys. Rev. Lett. 65, 551 (1990).

[3] T. N. Taddeucci et al., Phys. Rev. C 41, 2548 (1990).

[4] J. Rapaport et al., Phys. Rev. C 39, 1929 (1989).

[5] L. Ray and J. R. Shepard, Phys. Rev. C 40, 237 (1989).

[6] T. N. Taddeucci, private communication. 
[7] W. G. Love, K. Nakayama, and M. A. Franey, Phys. Rev. Lett. 59, 1401 (1987).

\section{Figure Captions}

Figure 1. Cross section and analyzing power for $494 \mathrm{MeV}{ }^{15} \mathrm{~N}(p, n){ }^{15} \mathrm{O}($ g.s. $)$ measured at NTOF. The curves are explained in the text.

Figure 2. Values of (a) $R^{2}$, (b) the $0^{\circ} \mathrm{GT}$ cross sections, and (c) the $0^{\circ} \mathrm{F}$ cross sections for ${ }^{14} \mathrm{C}(p, n){ }^{14} \mathrm{~N}$ at energies $100-800 \mathrm{MeV}$. The curves are explained in the text.
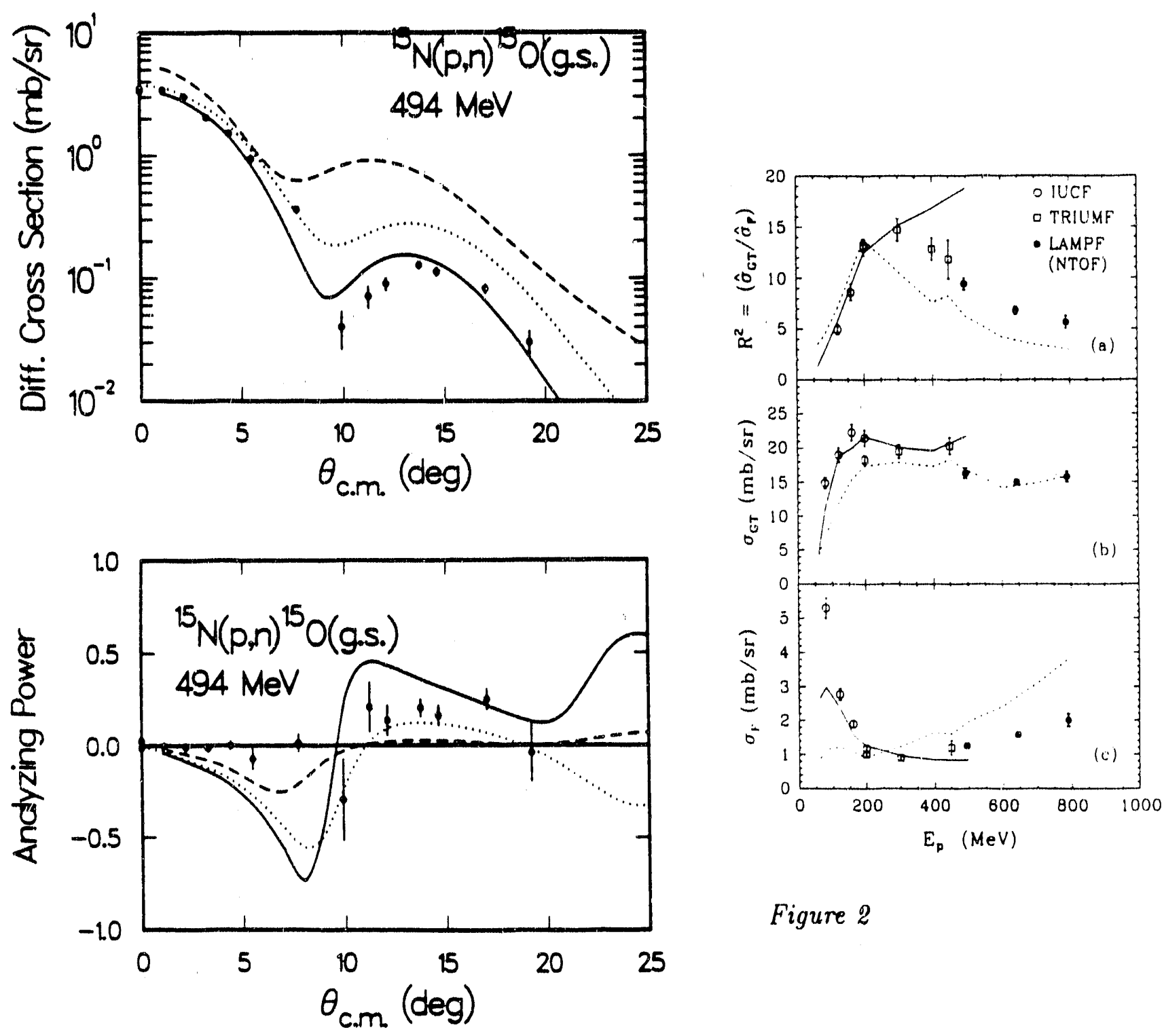

Figure 2

Figure 1 


\title{
4 Pion-Nucleus Experiments at LAMPF
}

\subsection{EXP 1023: Analyzing Powers for Pion Charge Ex- change on Polarized ${ }^{13} \mathrm{C}$}

\author{
(M. L. Barlett, G. W. Hoffmann, M. Purcell; ASU; NMSU; LANL; \\ Minnesota; Colorado; Maryland; J. Comfort, G. Kyle, spokesmen)
}

The pion-nucleon scattering amplitude has a spin-independent and a spin-orbit term. Each is complex and has isoscalar and isovector components. Models of pion-nucleus scattering generally build a theoretical description of the scattering process starting from this interaction, an assumed target nuclear density distribution, and include medium effects, etc. Of great interest is the study of the differences between the free pion-nucleon interaction and the "effective" pion-nucleon interaction experienced in the nuclear medium. An understanding of this "effective" interaction is one of the primary goals of pion-nucleus physics.

The pion-nucleus single-charge-exchange reaction (SCX) is directly sensitive to the isovector part of the effective pion-nucleon interaction. A considerable amount of SCX cross section data has been accumulated over the years, and these data are mainly sensitive to the spin-independent part of this piece of the interaction. SCX experiments with polarized nuclear targets may provide data which are useful in determining the effective isovector spin-dependent piece.

As part of a new program at LAMPF to exploit polarized nuclear targets in medium energy physics research, EXP 1023 measured analyzing powers $A_{y}$ for the $\left(\pi^{+}, \pi^{\circ}\right)$ SCX reaction on a polarized ${ }^{13} \mathrm{C}$ target at $T_{\pi^{+}}=163 \mathrm{MeV}$. The experiment spanned the center-of-momentum angular range $20^{\circ}-60^{\circ}$. The measurements were made at the Low Energy Pion Channel (LEP) at LAMPF with the LAMPF $\pi^{\circ}$ spectrometer. The polarized target was essentially the same as the one used in EXP 955 apart from different target geometry. Dummy target data and the development of peak fitting techniques allowed extraction of yields to the isobaric analog state (IAS) and combined yields to the $3 / 2^{-}, 5 / 2^{+}$doublet at $3.5 \mathrm{MeV}$.

The IAS data with theoretical curves are shown in Fig. 1 (Ref. 1). The solid curves are results of a distorted-wave impulse approximation (DWIA) calculation with a Cohen and Kurath wave function; the dashed curves are results of another DWIA calculation that used a Tiator wave function. The main difference in the results of these two calculations traces to the treatment of medium modifications to the effective pion-nucleon interaction (not to nuclear structure differences). There is reasonable qualitative agreement between experiment and theory (solid curves) in the region of the diffraction minima. The agreement is worst near $60^{\circ}$ where a problem is evident for both cross section and analyzing power. It is possible that the ${ }^{13} \mathrm{C}$ nuclear structure input requires configurations from beyond the $1 p$ shell. Theoretical work will continue to explore what new physics can be learned from 
these new pion-nucleus spin-dependent data.

\section{References}

[1] J. J. Görgen et al., Phys. Rev. Lett. (submitted).

\section{Figure Caption}

Figure 1. Cross sections and analyzing powers for the ${ }^{13} \overrightarrow{\mathrm{C}}\left(\pi^{+}, \pi^{\circ}\right){ }^{13} \mathrm{~N}(\mathrm{IAS})$ reaction at $163 \mathrm{MeV}$. The curves are results of DWIA calculations.

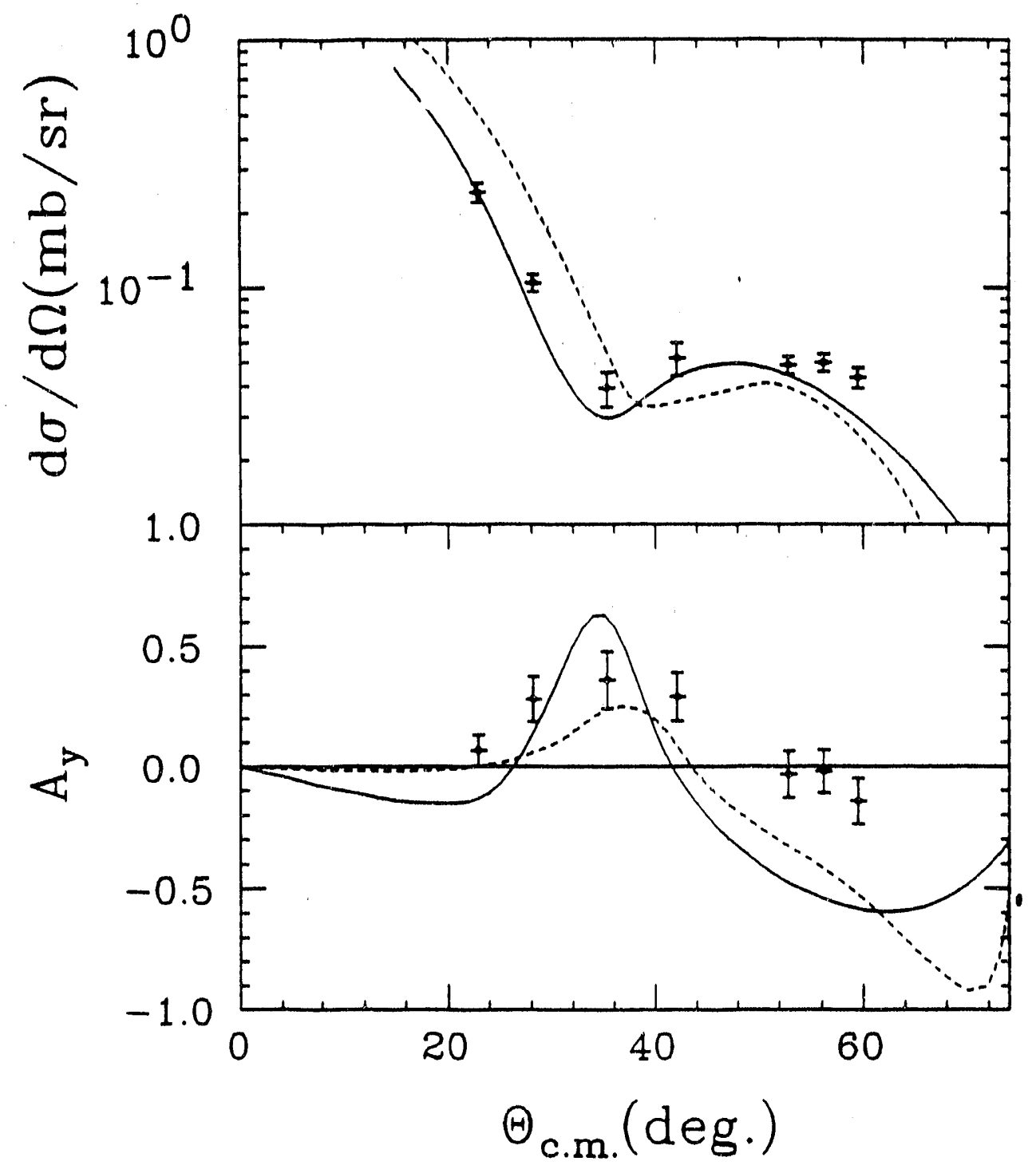




\subsection{EXP 1025: Pion Elastic/Inelastic Scattering from Po- larized ${ }^{13} \mathrm{C}$ in the Energy Region of the $[3,3]$ Reso- nance}

(M. Barlett, G. Hoffmann, M. Purcell; Minnesota; NMSU; ASU; LANL;

Penn; Colorado; Maryland; CSPU; G. Burleson, D. Dehnhard, spokesmen)

The general motivation for this experiment is much the same as that for EX.P 1023: to initiate an experimental program in pion-nucleus physics which utilizes polarized nuclear targets. The goal is to exploit the new spin degree of freedom of the target nucleus to study new parts of the pion-nucleon effective interaction.

EXP 1025 is a study of elastic and inelastic $\pi^{+} / \pi^{-}$scattering from ${ }^{13} \overrightarrow{\mathrm{C}}$ at energies around the $[3,3]$ resonance. The new observable, the analyzing power $A_{y}$, should provide data that further tests the available pion-nucleus scattering models, tests nuclear structure input used by the models, and leads to new information about $\pi$-nucleon "effective" interactions-especially the isoscalar spin-orbit piece.

The first run ${ }^{1}$ was in summer 1989 at the Low Energy Pion Channel (LEP) at LAMPF using the Large Acceptance Spectrometer (LAS) and essentially the same polarized target as developed for EXP 955 . The energy resolution of $3 \mathrm{MeV}$ was adequate to resolve elastic scattering. Differential cross sections $d \sigma / d \Omega$ and $A_{y}$ were measured for $T_{\pi^{+} / \pi^{-}}=132 \mathrm{MeV}, T_{\pi^{+}}=226 \mathrm{MeV}$, and $T_{\pi^{-}}=231 \mathrm{MeV}$. Surprisingly, analyzing powers significantly different from zero were seen only for the $132 \mathrm{MeV} \pi^{-}$data (see Fig. 1). The analyzing powers for the $231 \mathrm{MeV}$ and $226 \mathrm{MeV}$ data are almost consistent with zero over the entire angular range spanned by the data. In addition, analyzing powers were measured at momentum transfers near the second minimum of $d \sigma / d \Omega$ at several energies across the $[3,3]$ resonance. Here again, the analyzing powers, contrary to expectations, are observed to be small (see Fig. 2).

The curves shown in Fig. 1 are results of a variety of distorted wave impulse approximation calculations. ${ }^{1}$ The solid curves resulted from calculations employing a first-order optical potential generated from $\pi$-nucleon amplitudes and CohenKurath wave functions. The results labelled Mach (long-short dashed curves) are from similar calculations but include a phenomenological $\rho^{2}$ term. The shortdashed curves are from calculations that used the same model that produced the long-short dashed curves except that the wave function of Tiator was used to generate the optical potential. This result suggests strong dependence of the analyzing power on nuclear structure.

It is interesting that none of the calculations qualitatively fit the analyzing power data for $\pi^{+} / \pi^{-}$at $132 \mathrm{MeV}$, especially near the second minimum in the cross section, near $100^{\circ} \mathrm{c} . \mathrm{m}$. The standard nuclear structure models also fail to reproduce the measured magnetic form factor of ${ }^{13} \mathrm{C}$ at $\mathrm{q} \geq 1.6 \mathrm{fm}^{-1}$.

The second phase of the experiment was recently completed on the EPICS 
channel at LAMPF. Data were taken for $\pi^{+} / \pi^{-}$at $162 \mathrm{MeV}$ over the laboratory angular range $30^{\circ}-90^{\circ}$. The intention is to obtain some inelastic cross section and analyzing power angular distributions from the data taken during this run. The task is complicated by the disappointing $1.2 \mathrm{MeV}$ overall experimental resolution that is presently achieved during the offline analysis.

Experimental and theoretical work will continue to explore what new physics can be leared from these new and interesting $\pi$-nucleus analyzing power data.

\section{References}

[1] Yi-Fen Yen et al., Phys. Rev. Lett. (to be submitted).

\section{Figure Captions}

Figure 1. Cross sections and analyzing powers for $132 \mathrm{MeV} \pi^{+} / \pi^{-}$elastic scattering from ${ }^{13} \overrightarrow{\mathrm{C}}$ at $132 \mathrm{MeV}$. The curves are results from a vas iy of DWIA calculations.

Figure 2. Analyzing powers for $\pi^{-}$elastic scattering from ${ }^{13} \overrightarrow{\mathrm{C}}$ across the $[3,3]$ resonance at momentum transfers near the second minimum of the differential cross sections. 
Figure 1
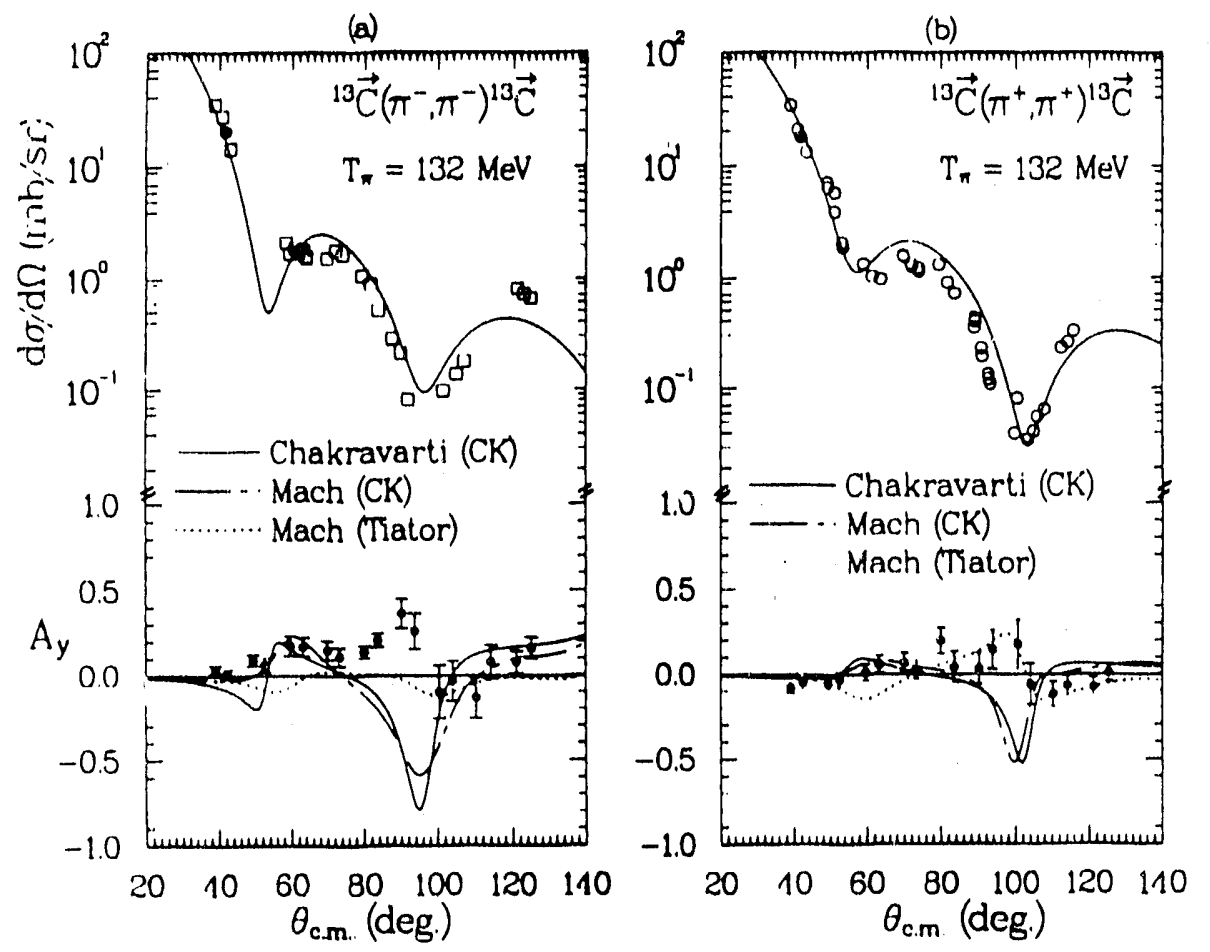

Figure 2

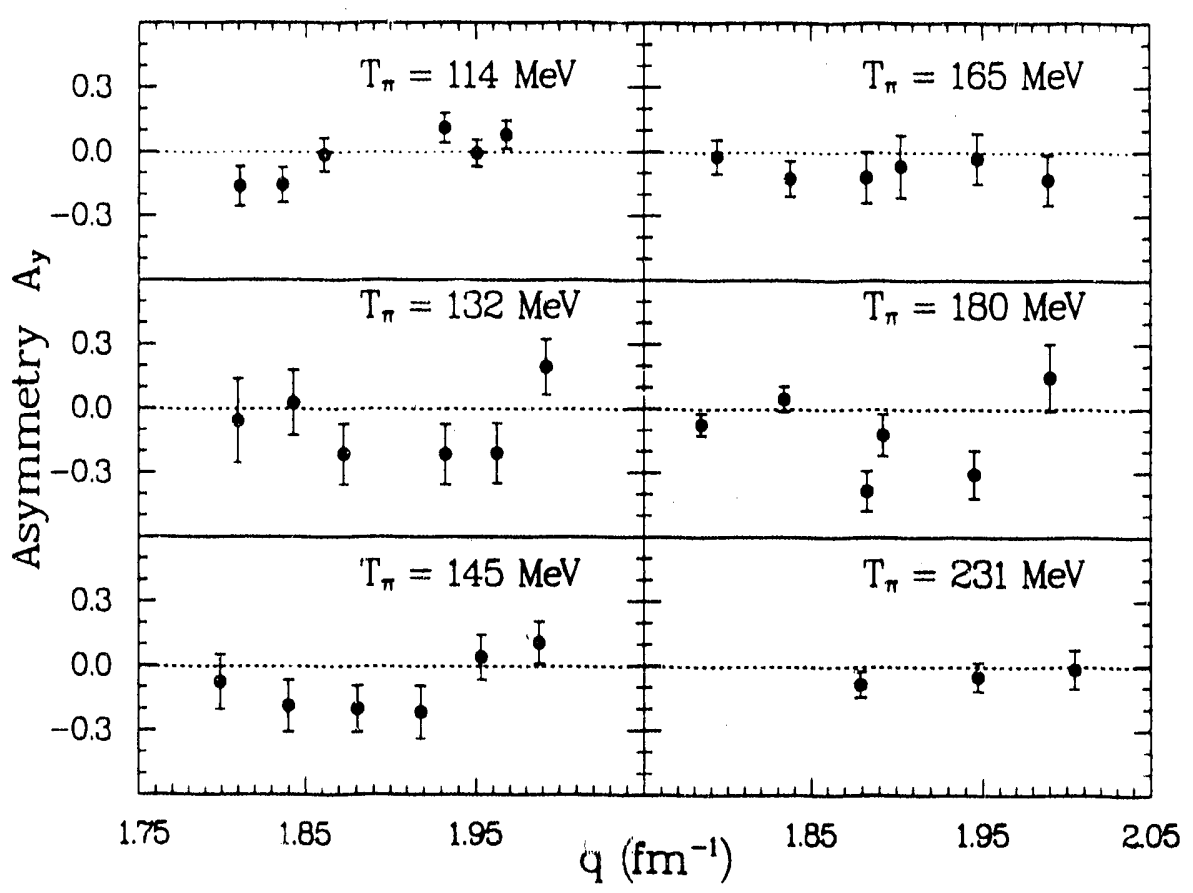




\section{Experiments at Brookhaven National Labo- ratory}

\subsection{BNL EXP 781: Search for Radiative Transitions in the Hypernucleus ${ }_{\Lambda}^{10} \mathrm{~B}$}

(M. L. Barlett, G. W. Hoffmann, E. C. Milner; BNL; Carnegie-Mellon;

Columbia; MIT; Houston; Osaka; Vassar College;

M. May and M. Deutsch, spokesmen)

The intent of BNL EXP 781 was to search for radiative transitions in the pshell hypernucleus ${ }_{\Lambda}^{10} \mathrm{~B}$ corresponding to $\mathrm{M} 1$ spin-flip transitions between members of the doublet formed by coupling an s-shell $\Lambda$ hyperon to the (A-1) nuclear core. ${ }^{1}$ For low-energy excitations of ${ }_{\Lambda}^{10} \mathrm{~B}$ the simplest single particle configuration is $(1 s)_{N}^{4}(1 p)_{N}^{5}(1 s)_{\Lambda}$. The spectrum of states associated with the $(\mathrm{A}-1)$ core will, in general, be split into a series of doublets due to the spin-dependent, $\Lambda$-core interaction. Hypernucleus shell-model calculations ${ }^{2}$ using phenomenological $\Lambda \mathrm{N}$ spin-dependent effective interactions predict doublet M1 transition energies in the range 0.1 to $1 \mathrm{MeV}$.

The experiment was conducted at the Brookhaven National Laboratory using the low-energy separated kaon beam at the Alternating Gradient Synchrotron (AGS). The observed reaction was ${ }^{10} \mathrm{~B}\left(\mathrm{~K}^{-}, \pi^{-} \gamma\right){ }_{\Lambda}^{10} \mathrm{~B}$ using $800 \mathrm{MeV} / \mathrm{c}$ kaons and covering the 4 to $12 \mathrm{MeV} \Lambda$ hyperon binding energy range in ${ }_{\Lambda}^{10} \mathrm{~B}$. The reaction pions were detected with a QQDQQ spectrometer system ("Moby Dick"). Thin target $\left(3.9 \mathrm{~g} / \mathrm{cm}^{2}\right)$ runs were made to experimentally identify bound states in ${ }_{\Lambda}^{10} \mathrm{~B}$ and establish production cross sections. The radiative decay measurements were made using a thick target $\left(12.3 \mathrm{~g} / \mathrm{cm}^{2}\right)$. Camma-rays were detected with an assembly of six germanium detectors mounted above and below the ${ }^{10} \mathrm{~B}$ target.

The $\gamma$-ray spectrum in coincidence with the ${ }_{\Lambda}^{10} \mathrm{~B}$ bound state region is shown in Figs. 1 and 2. The solid line in Fig. 1 shows the expected total number of events predicted for the $2^{-}$to $1^{-}{ }_{\Lambda}^{10} \mathrm{~B}$ ground state doublet transition. ${ }^{1}$ The dashed line in this figure represents the $5 \%$ confidence limit for that transition. There are no observable peaks which approach the predicted size in this region of the bound state spectrum. Fig. 2 shows an expanded portion of the spectrum in Fig. 1, this being the predicted region for the doublet transition according to Ref. 2. A possible $2^{-}$to $1^{-}$(g.s.) M1 transition is therefore ruled out in this energy range. These results are in sharp disagreernent with the predictions of Ref. 2 .

\section{References}

[1] R. E. Chrien et al., Phys. Rev. C 41, 1062 (1990). 
[2] D. J. Millener, A. Gal, C. B. Dover and R. H. Dalitz, Phys. Rev. C 31, 499 (1985).

\section{Figure Captions}

Figure 1. The spectrum observed for ${ }^{10} \mathrm{~B}\left(\mathrm{~K}^{-}, \pi^{-} \gamma\right){ }_{\Lambda}^{10} \mathrm{~B}$. The solid line indicates the expected event total predictcd for the ${ }_{\Lambda}^{10} \mathrm{~B}$ ground-state doublet transition. The dashed line represents the $5 \%$ confidence limit for that transition.

Figure 2. An expanded portion of Fig. 1.

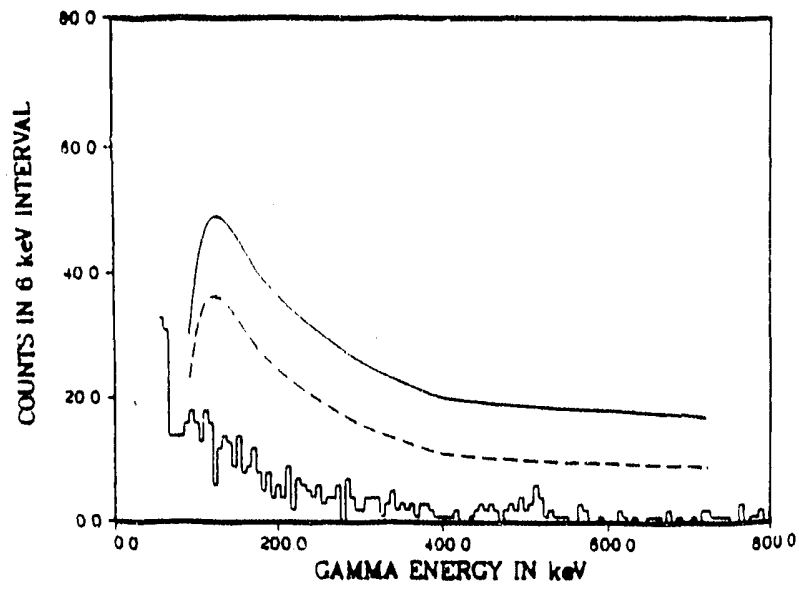

Figure 1

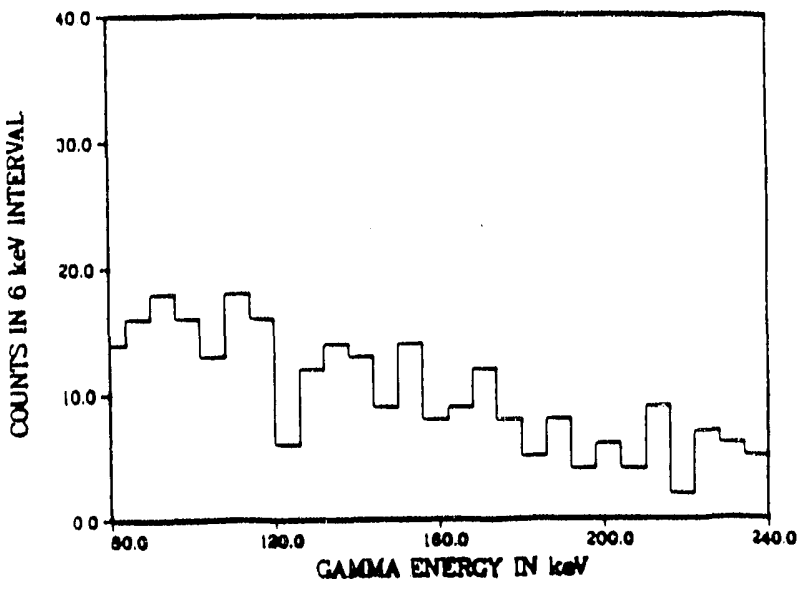

Figure 2 


\subsection{BNL EXP 791: Search for $K_{L}^{0} \rightarrow \mu e, K_{L}^{0} \rightarrow e e$}

(G. Hoffmann, J. McDonough; UCI; UCLA; LANL; Stanford; Temple;

Texas; William and Mary; R. Cousins, W. Molzen, spokesmen)

The present description of the fundamenti.l particles and their electroweak and strong interactions is the Minimal Standard Model (MSM). Although this model has never been shown to be wrong, because of its many arbitrary parameters, it is suspected to be incomplete. Measurements performed in recent years make it clear that this incompleteness must originate with physics at very large mass scales. Rare decay physics probes some of these higher mass scales.

In the MSM the decay $K_{I}^{0} \rightarrow \mu e$ is forbidden by conservation of electron and muon additive quantum numbers. Its observation would be the first direct evidence of physics beyond the MSM. The decays $K_{L}^{0} \rightarrow \mu \mu$ and $K_{L}^{0} \rightarrow e e$ are permitted in the MSM, but are highly suppressed. $K_{L}^{G} \rightarrow e e$ has not been seen, and observation of a branching ratio for this process that is significantly above MSM predictions would ulso be evidence for new physics.

BNL EXP 791 is a high sensitivity search for $K_{L}^{0} \rightarrow \mu e$ and $K_{L}^{0} \rightarrow e e$. The spectrometer, located on the B5 beam line of the Alternating Giadient Synchrotron facility of the Brookhaven National Laboratory, is shown schematically in Fig. 1. This spectrometer provides precise tracking with good (redundant) particle identification. Very high rate capability is achieved using custom buiit front-end electronics, a large parallel readout architecture, and a multi-level trigger system utilizing SLAC 3081/E emulators.

The experiment has had three production runs spanning $1988-1990$. We joined the collaboration in 1989 and participated in the 18 week 1990 run. In 1989 our intuial contrihution to the experiment was the design and prototyping of an efficient, low mass, "upstream trigger hodoscope" for use between the two analyzing magnets shown in Fig. 1. After the 1990 production run we were instrumental in testing a beam dump installed in the second dipole magnet to investigate whether such a concept could be utilized for a new, factor of 10 type improvement, experiment. We assumed considerable responsibility for this plug test and the interpretation of the results. Based on the results of the plug test we are presently designing a new plug for additional tests to be made in early 1991.

The first stage (and most computer intensive) of the offline analysis of the 1990 data is now complete. This analysis involved processing all data tapes using event reconstruction algorithms. From this analysis we can already give a preliminary sensitivity of the 1990 data set for a singi. $K_{L}^{0} \rightarrow \mu e$ event. The single event sensitivity is about $3 \times 10^{-11}$. When all three data sets are combined it is expected that this single event sensitivity will be about $1.5 \times 10^{-11}$ (implying a final limit $\leq 4 \times 10^{-11}$ at the $90 \%$ confidence level).

In addition to providing the world's best limit on $K_{L}^{0} \rightarrow \mu e$, the experiment has accimulated a large number of $K_{L}^{0} \rightarrow \mu \mu$ events. It is anticipated that the 
combined 1988 - 1990 runs will give a total number of about 600 events (350 from the 1990 run). This will imply knowledge of the branching ratio to about $5 \%$.

\section{References}

[1] C. Mathiazhagan et al., Phys. Rev. Lett. 63, 2185 (1989).

[2] C. Mathiazhagen et al., Phys. Rev. Lett. 63, 2181 (1989).

\section{Figure Caption}

Figure 1. Schematic drawing of the kaon beam line and detection apparatus for BNL EXP 791.

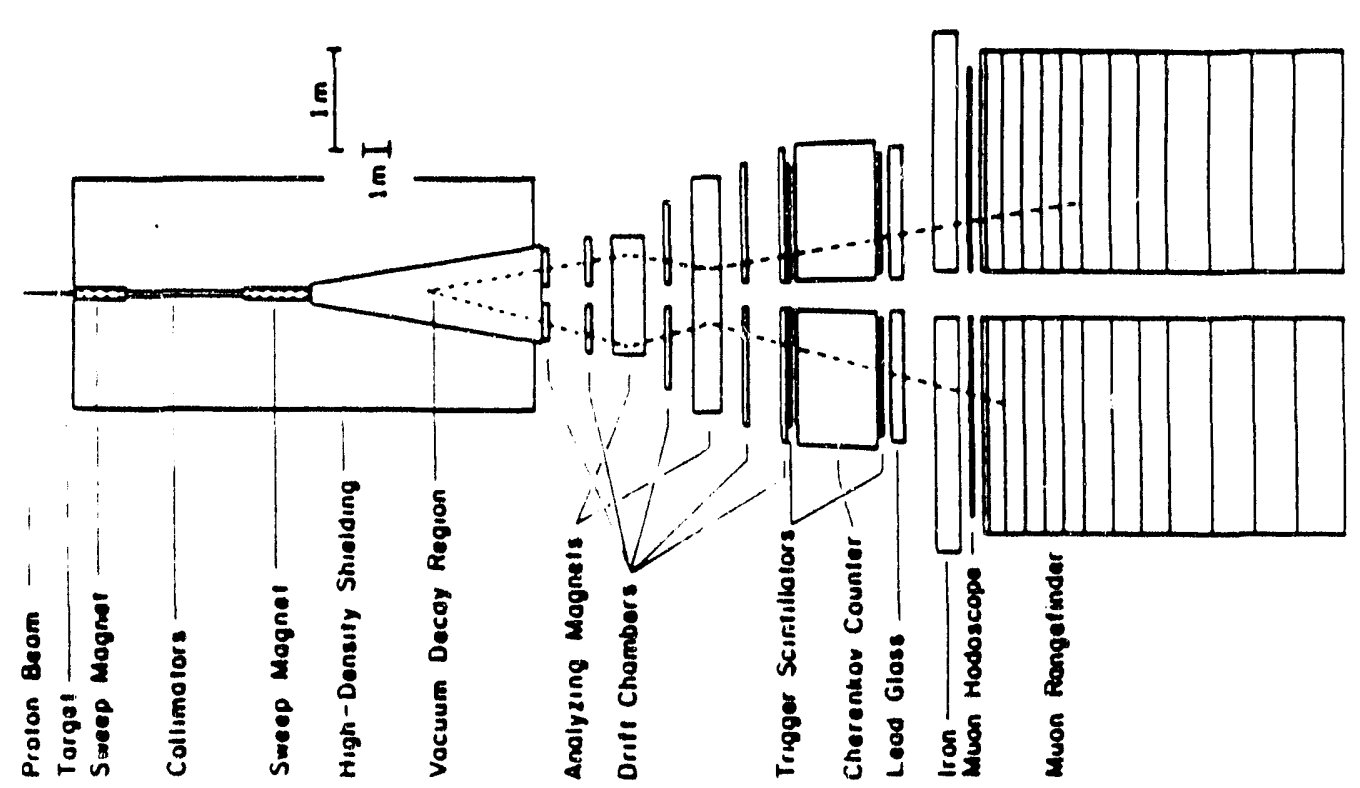




\section{Experiments at Fermilab}

\subsection{FNAL EXP 772: Study of the Nuclear Antiquark Sea Via $p+\mathrm{N} \rightarrow$ Dimuons}

(M. L. Barlett, G. W. Hoffmann; LANL; FNAL; Illinois; Northern

Illinois; SUNY at Stony Brook; and Case Western Reserve;

J. Moss, spokesman)

The European Muon Collaboration (EMC) was first to observe a modification of the quark distribution in nuclei compared to that in free nucleons. ${ }^{1}$ Many explanations have been offered but, as yet, a generally accepted consensus on the origin of this effect has not emerged. For the region of fractional quark momenta $0.3 \leq x \leq 1.0$ the EMC data are most straightforwardly accounted for with Fermi motion and nuclear binding effects. The region $x \leq 0.1$ remains active both experimentally and theoretically.

An independent measure of the quark structure of nuclei is provided by continuum dimuon production in high energy hadron-nucleus collisions via the Drell-Yan (DY) process, $q+\bar{q} \rightarrow \ell^{+} \ell^{-}$. For $x_{F} \geq 0.2$ this process is dominated by beam quark - t,arget antiquark annihilation and is thus sensitive to the nuclear antiquark distribution. This complements the deep inelastic lepton scattering which is sensitive to both quark and antiquark distributions in nuclei.

In FNAL EXP 772 a precise measurement of the A dependence of dimuon production induced by $800 \mathrm{GeV}$ protons from targets of ${ }^{2} \mathrm{H}, \mathrm{C}, \mathrm{Ca}, \mathrm{Fe}$ and $\mathrm{W}$ was made. ${ }^{2-4}$ Over 450,000 muon pairs with invariant mass $\mathrm{M} \geq 4 \mathrm{GeV}$ were recorded. The experiment was run in the Meson Easi beam line at Fermilab using the beam line, spectrometer, and beam stop of FNAL EXP 605 (with modifications). ${ }^{3}$ Systematic errors were minimized by interchanging the solid targets with the ${ }^{2} \mathrm{H}$ target every few minutes. Total systematic errors in the data ratios are less than $2 \%$.

Fig. 1 shows a composite dimuon yield spectrum for all targets revealing very clear $J / \psi$ and $\psi^{\prime}$ peaks, the broad DY continuum from $4 \leq \mathrm{M} \leq 9 \mathrm{GeV}$ and a partial resolution of the $\Upsilon$ states. Fig. 2 shows the ratios of integrated yields per nucleon for the nuclear targets relative to deuterium for the $J / \psi, \psi^{\prime}$ and $\Upsilon$ resonances and the DY continuum. The strong $A$ dependence of the quarkonium resonances contrasts sharply with the A independence of the DY continuum. Fig. 3 shows the ratios of DY yield per nucleon for each target to ${ }^{2} \mathrm{H}$ as a function of $x_{t}$ for muon pairs with positive $x_{F}(0.26)$. The curves shown for $\mathrm{Fe} /{ }^{2} \mathrm{H}$ are predictions of various models of the EMC effect. No nuclear mass dependence is found for $x_{t}>0.1$ but a slight suppression at $x_{t}<0.1$ (shadowing) is observed for the heavier targets. Models of the EMC effect which postulate a significant pion excess or hadron clusters are apparently ruled out. 'The strong mass dependence of the $J / \psi, \psi^{\prime}$ and $\Upsilon \Upsilon$ states cannot be explained by small- $x$ shadowing or hadronic attenuation models. 


\section{References}

[1] J. J. Aubert et al., Phys. Lett. B 163, 275 (1983).

[2] M. R. Adams et al., in Contributions to the Int. Conf. on Particles and Nuclei, PANIC XII, Mass. Inst. Tech., 1990, p. VIII-1 .

[3] D. M. Alde et al., Phys. Rev. Lett. 64, 2479 (1990).

[4] D. M. Alde et al., Phys. Rev. Lett. (1990) (submitted).

\section{Figure Captions}

Figure 1. Dimuon event distribution measured in E772.

Figur: 2 . Measured A-dependence of integrated yields for $J / \psi, \psi^{\prime}, \Upsilon(1 s)$ and Drell-Yan production.

Figure 3. Ratios of the Drell-Yan dimuon yield per nucleon for positive $x_{F}$. The curves are discussed in the text. 

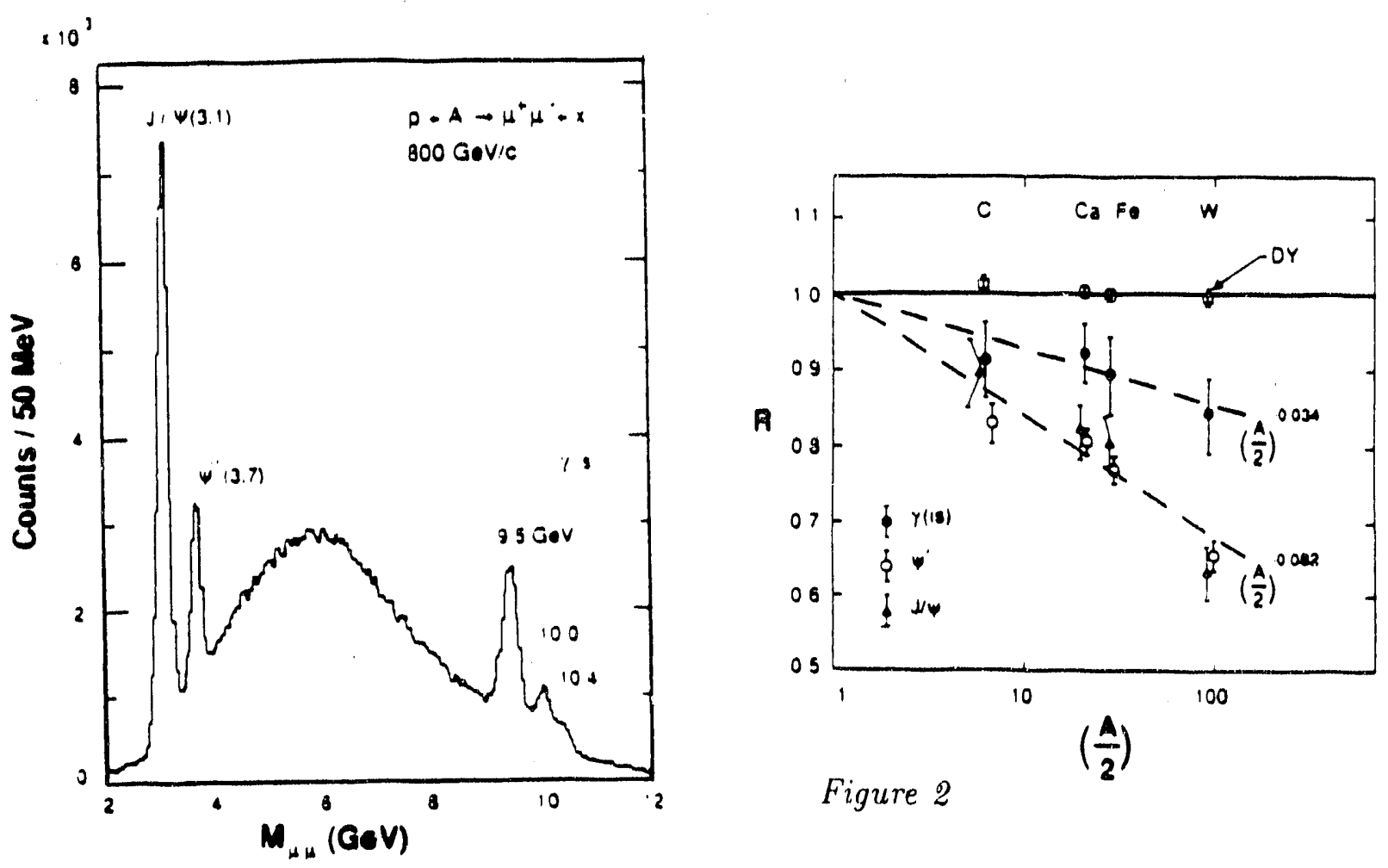

Figure 1

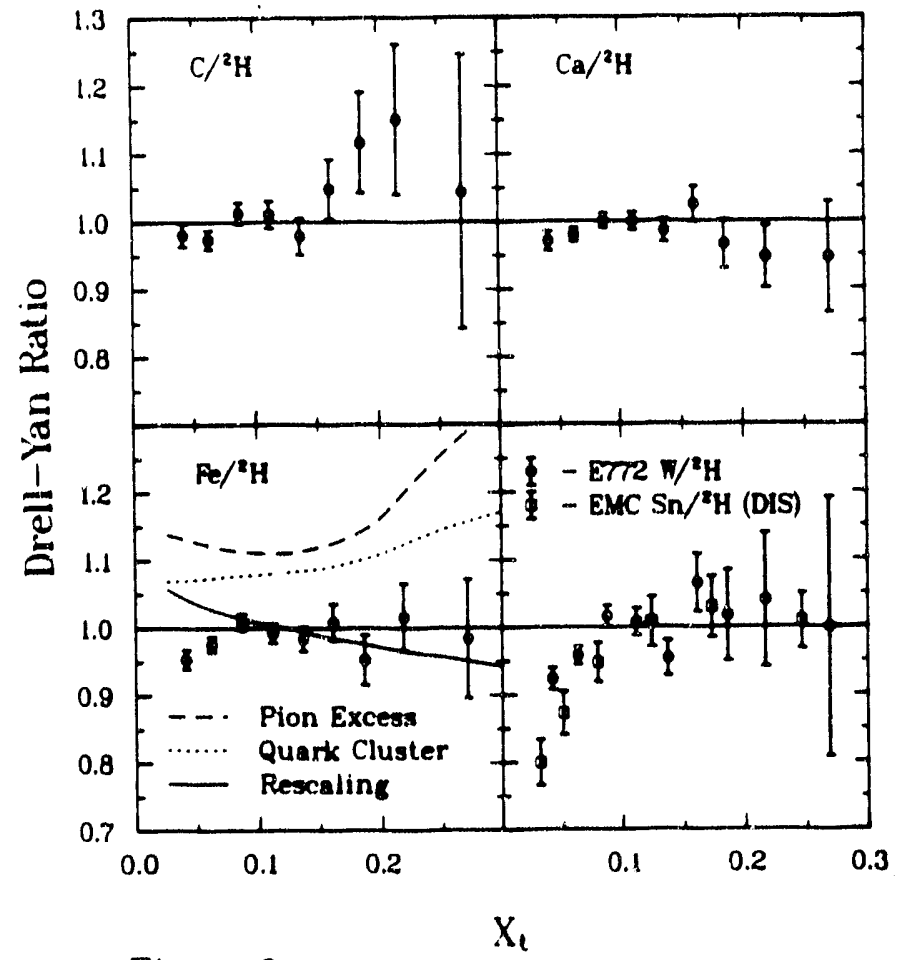

Figure 3 


\title{
7 Polarized Nuclear Target Development Work
}

\author{
(G. Hoffmann, M. Barlett, M. Purcell, S. Worm, D. Read; LANL; ANL)
}

Because of broad interest in exploring the new physics that can be learned through use of polarized nuclear targets in medium energy scattering experiments, and as an initial step in the realization of such experiments, we have polarized ${ }^{13} \mathrm{C}$ and ${ }^{19} \mathrm{~F}$ using dynamic nuclear polarization techniques (DNP) ${ }^{1,2}$ The ${ }^{13} \mathrm{C}$ work led to the development of the polarized target constructed at $\mathrm{LAMPF}^{3}$ and used for experiments 955, 1023, and 1025. This target was fabricated from one of the LAMPF setups originally designed to provide a polarized ${ }^{1} \mathrm{H}$ target for the LAMPF nucleon-nucleon program. Physically this setup (cryostat plus supporting equipment such as vacuum pumps, dewars, NMR hardware, microwave hardware, etc.) is large and considerable manpower and overhead is required in its use for $p+$ nucleus and $\pi+$ nucleus scattering experiments.

For the past 15 years the PSI group in Switzerland, headed by Prof. S. Mango, has been developing compact ${ }^{3} \mathrm{He}$ evaporation $/{ }^{3} \mathrm{He}-{ }^{4} \mathrm{He}$ dilution refrigerators ${ }^{4}$ for use with the polarized proton/deuteron experiments performed at PSI. Such compact setups would be ideal for the programs that seem to be starting up at LAMPF with polarized nuclear targets. Because of our longer term interest in performing medium energy experiments which utilize polarized nuclear targets, we have begun construction of a copy of the PSI target setup. Schematic diagrams of the cryostat, the ${ }^{3} \mathrm{He}$ evaporation unit, and the ${ }^{3} \mathrm{He}^{-4} \mathrm{He}$ dilution refrigerator unit are shown in Figs. 1-3. PSI provided us with a complete set of engineering drawings for the system. They are also consulting with us concerning this technically difficult project.

The cryostat construction is complete and approximately $50 \%$ of the ${ }^{3} \mathrm{He}$ evaporation unit has been made at this time. All components for the $70 \mathrm{GHz}$ microwave system required for DNP (at a magnetic field of $2.5 \mathrm{~T}$ ) have been acquired. We have also acquired most of the components required for the NMR system. The microvax-CAMAC controlled NMR system developed for EXP 955 will be updated and used for the new system. We are presently procuring the ${ }^{3} \mathrm{He}$ purnping system, the ${ }^{3} \mathrm{He}$ storage system, as well as a $2.5 \mathrm{~T}$ superconducting split-coil magnet for the target.

The intention is that the setup will serve to test new materials for polarization feasibility as well as serve as the actual target for experiments at LAMPF and elsewhere.

The target is designed to be service and user friendly. The sample holding device can be removed or inserted into the cryostat at $4 \mathrm{~K}$. PSI experience is that the target can be installed in the time period of one day. The comparable time period for the LAMPF setup is one month. The availability of the new PSI designed target will greatly benefit future polarized nuclear target experiments at LAMPF. 


\section{References}

[1] J. J. Jarmer et al., Nucl. Inst. and Methds. A250, 576 (1986).

[2] D. Hill et al., Nucl. Inst. and Methds. A277, 319 (1989).

[3] S. I. Penttilä et al., Eighth Int. Symp. of High-Energy Spin Physics, American Institute of Physics Conf. Proc. No. 187, New York, 1989, p. 1281.

[4] B. van den Brandt, J. A. Konter, and S. Mango, Nucl. Inst. and Methods. A289, 526 (1990).

\section{Figure Captions}

Figure 1. Schematic of PSI standard cryostat with central tube for ${ }^{3}$ He evaporation insert (Fig. 2) or dilution unit (Fig. 3).

Figure 2. Schematic of PSI ${ }^{3}$ Hie evaporation unit and sample holding device.

Figure 3. Schematic of PSI ${ }^{3} \mathrm{He}^{-4} \mathrm{He}$ dilution refrigerator unit with corresponding sample holding device.

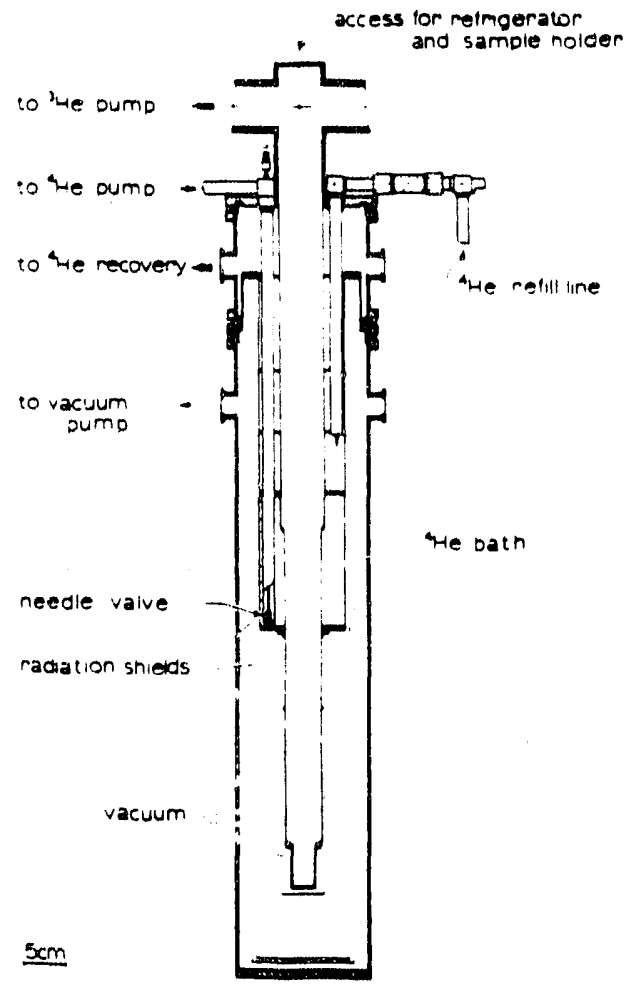

Figure 1

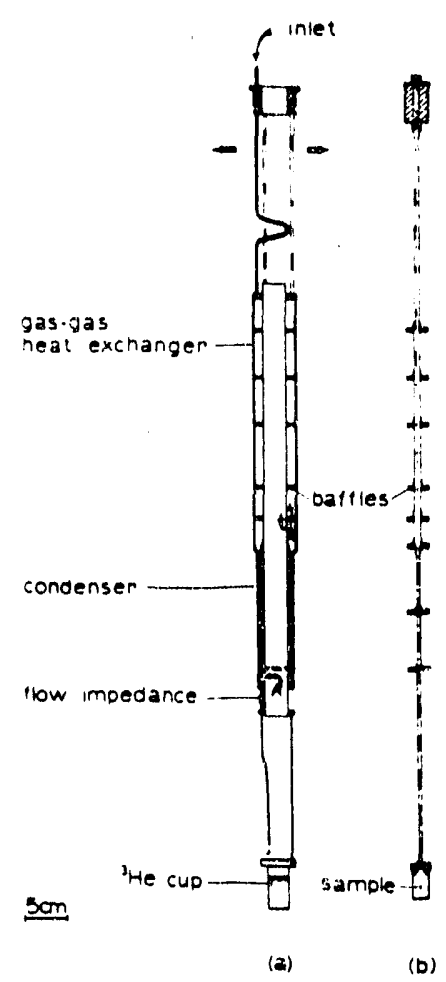

Figure 2

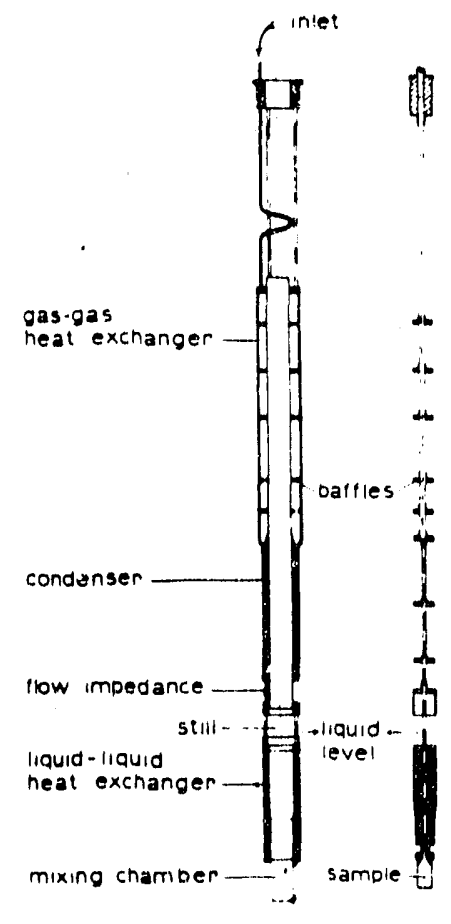

(a) (b)

Figure 3 


\section{Theoretical Work}

\subsection{Spin-Flip Cross Sections for ${ }^{13} \mathrm{C}(\vec{p}, \vec{n}){ }^{13} \mathrm{~N}(\mathrm{~g} \cdot \mathrm{s})$ at 500 $\mathrm{MeV}$}

(L. Ray and J. R. Shepard (University of Colorado))

During the last few years $(p, n)$ charge exchange (CEX) data have been taken at the Neutron Time-of-Flight (NTOF) facility at LAMPF. The first set of data obtained was for the reaction ${ }^{15} \mathrm{~N}(\vec{p}, n){ }^{15} \mathrm{O}$ (g.s.) at $500 \mathrm{MeV}$. Analyses have shown that the analyzing power cannot be explained by current theoretical models. ${ }^{1}$

Understanding this failure is made difficult by the fact that each component of the isovector NN effective interaction contributes significantly to the cross section, analyzing power, and polarization transfer $\left(D_{i j}\right)$ observables in a mixed and complicated manner. We have developed a method for attacking this problem by utilizing certain linear combinations of the $(\vec{p}, \vec{n})$ polarization transfer observables which allows one to focus attention on the individual dynamical elements of the reaction model. ${ }^{2}$ The method was illustrated with relativistic distorted wave impulse approximation ${ }^{3}$ (RDWBA) calculations for the ${ }^{13} \mathrm{C}(\vec{p}, \vec{n})^{13} \mathrm{~N}(\mathrm{~g} . \mathrm{s})$ reaction at $500 \mathrm{MeV}$. In this approach the RDWBA transition amplitude is determined by the relativistic distorted waves, the $1 p_{\frac{1}{2}}$ valence bound state wave function, and the isovector components of the nucleon-nucleon (NN) Lorentz invariant amplitudes. Both Fermi (F) and Gamow-Teller (GT) transitions contribute. Assuming isospin invariance the transition amplitude is given $b^{2}$

$$
F_{C E X}=A_{F}+B_{F} \sigma_{n}^{p}+A_{G T} \sigma_{n}^{t}+B_{G T} \sigma_{n}^{p} \sigma_{n}^{t}+C_{G T} \sigma_{p}^{p} \sigma_{p}^{t}+D_{G T} \sigma_{q}^{p} \sigma_{q}^{t},
$$

where superscripts $(p)$ and $(t)$ refer to the projectile nucleon and target nucleus, respectively. The unit vectors $\hat{n}, \hat{p}$, and $\hat{q}$ (denoted by subscripts) correspond to directions normal to the scattering plane and perpendicular and parallel to the momentum transfer, respectively. The longitudinal and transverse spin-flip cross sections, obtained from the above amplitudes by the following: ${ }^{2}$

$$
\sigma S_{F P}^{L}=\left|D_{G T}\right|^{2}, \quad \sigma S_{F P}^{T}=\left|C_{G T}\right|^{2},
$$

depend predominantly on just the isovector-pseudoscalar and isovector-tensor components of the Lorentz invariant interaction as shown in Figs. 1 and 2. A non-spinflip cross section, $\sigma_{0}=\left|A_{F}\right|^{2}+\left|A_{G T}\right|^{2}$, is predominantly sensitive to part of the Fermi interaction at forward angles. By studying these particular cross sections, along with $d \sigma / d \Omega, A_{y}$, and $D_{i j}$, the different parts of the $N N$ isovector effective interaction in combination with the structure of the valence nucleon wave function can be studied one at a time in an optimum fashion.

It is intended that analyses of this type will provide an important tool for diagnosing the apparent inadequacies with the theoretical description of intermediate energy $(p, n)$ CEX from odd-mass nuclei. 


\section{References}

[1] D. Ciskowski et al., Bull. Am. Phys. Soc. 33, 1583 (1988); D. Ciskowski, Ph. D. thesis, The University of Texas at Austin, 1989; T. N. Taddeucci, private communication.

[2] L. Ray and J. R. Shepard, Phys. Rev. C 40, 237 (1989).

[3] L. Ray et al., Phys. Rev. C 37, 1169 (1988).

\section{Figure Captions}

Figures 1 and 2. RDWBA predictions for the transverse and longitudinal spin-flip cross sections. The solid curves (same in each respective figure) represent calculations assuming the pseudovector (PV) form for the $N N$ interaction and a relativistic lower component, $\lambda^{R e l}$, for the valence nucleon wave function. In the upper portion the dotted and dashed curves are from calculations that assumed the PV and pseudoscalar (PS) forms, respectively, where the nonrelativistic limit $f$ the valence nucleon lower component, $\lambda^{N R}$, was assumed. In the middle portion the dashed and dashed-dotted curves represent RDWBA calculations with the PS and tensor components of the NN interaction omitted, respectively. The dashed and dashed-dotted curves in the lower portion are similar except that the axial-vector and space-like vector components were omitted, respectively.
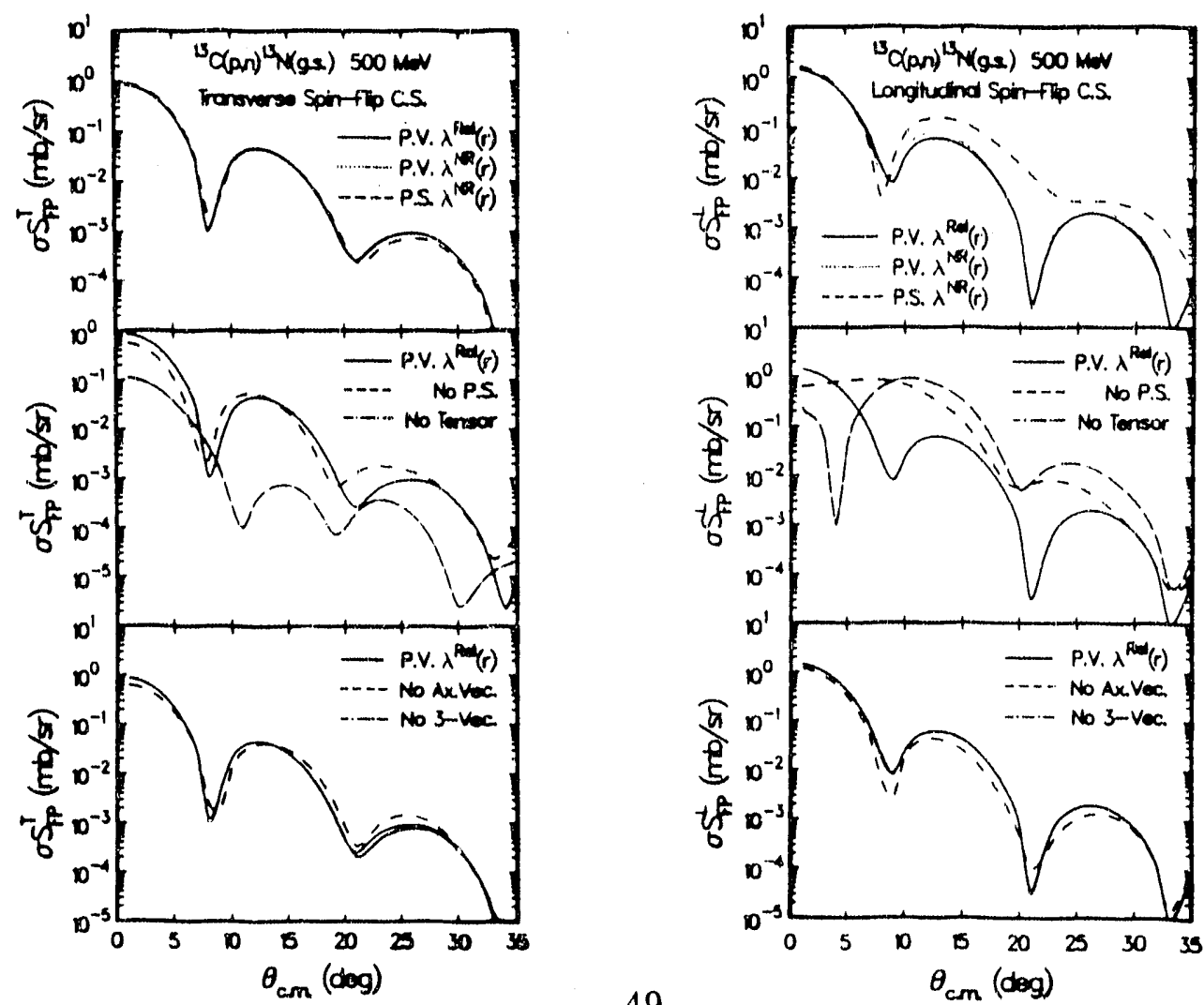


\title{
8.2 Multiple Scattering Description of Intermediate En- ergy Deuteron-Nucleus Scattering
}

\author{
(L. Ray)
}

Recent deuteron-nucleus $(d A)$ elastic scattering data from Saturne ${ }^{1,2}$ have dramatized the need for theoretical models for composite projectile - nucleus scattering which are appropriate for intermediate energy, which are based on microscopic, many-body theory, and which are readily calculable. In the past, studies of $d A$ elastic scattering have been carried out at low energies and have relied on the nonrelativistic folding model derived by Watanabe. ${ }^{3}$ It is important to realize that this model is based on an effective Hamiltonian for the $d A$ many-body system which uses nucleon-nucleus optical potentials and will therefore omit a number of multiple scattering processes. These can only be properly included by a microscopic reduction of the $(A+2)$-body problem.

In this work ${ }^{4}$ the nonrelativistic multiple scattering formalism of Watson ${ }^{5}$ and $\mathrm{KMT}^{6}$ was applied to $d A$ elastic scattering. An optical potential was obtained through second-order in the elemental nucleon-nucleon (NN) $t$ matrix and was organized such that the usual Watanabe folding potential appears as the leading term. This latter folding potential consists of a convolution of the nucleon-nucleus optical potentials, evaluated at half the deuteron incident kinetic energy, with the square of the s-state wave function of the deuteron. Corrections arising from deuteron-target nucleon double scattering, the deuteron $d$ state, virtual deuteron break-up, additional nuclear medium effects, additional target nucleon correlation effects, and possible relativistic effects were explicitly identified. These corrections arise even when accurate, well understood nucleon-nucleus optical potentials are employed in the folding model. They are of the same order in the NN $t$ matrix as the deuteron break-up corrections which are often included in calculations. ${ }^{7}$ Most of these corrections are small, however the additional medium effects are comparable to the break-up corrections. ${ }^{7}$ The leading term in the optical potential was obtained from Dirac phenomenolcgy for $p+{ }^{40} \mathrm{Ca}$ at $362 \mathrm{MeV}$.

Fredictions for $d+{ }^{58} \mathrm{Ni}$ at $700 \mathrm{MeV}$ using the lowest-order, folding potential are shown in Fig. 1 in comparison with data. ${ }^{1}$ Predictions for $d+{ }^{40} \mathrm{Ca}$ at $700 \mathrm{MeV}$ including corrections due to the $d$ state of the deuteron, target-nucleon - deuteron double scattering, and correlation effects beyond that accounted for in the nucleonnucleus phenomenology, are indicated by the solid curves in Fig. 2. The effects of the additional medium corrections are shown by the dashed curves in Fig. 2. Overall, the theoretical description of the data is fairly good. Virtual deuteron break-up corrections should further improve the descriptions of these data, particularly the differential cross sections.

The results of this work demonstrate that nonrelativistic multiple scattering approaches provide an efficient model for organizing the complex, deuteron-nucleus scattering problem into a manageable, effective one-body problem which is readily 
amenable to numerical analysis.

\section{References}

[1] N. Van Sen et al., Phys. Lett. B 156, 185 (1985).

[2] N. Van Sen et al., Nucl. Phys. A464, 717 (1987).

[3] S. Watanabe, Nucl. Phys. 8, 484 (1958).

[4] L. Ray, Phys. Rev. C 38, 2743 (1988).

[5] K. M. Watson, Phys. Rev. 89, 575 (1953).

[6] A. K. Kerman, H. McManus, and R. M. Thaler, Ann. Phys. (N.Y.) 8, 551 (1959).

[7] M. Yahiro et al., Prog. Theo. Phys. Suppl. 89, 32 (1986); Phys. Lett. B 182, 135 (1986).

\section{Figure Captions}

Figure 1. Predictions for $d+{ }^{58} \mathrm{Ni}$ at $700 \mathrm{MeV}$ are compared with data.

Figure 2. Predictions (solid curves) for $d+{ }^{40} \mathrm{Ca}$ at $700 \mathrm{MeV}$ which include corrections due to $d$ state of deuteron, double scattering, and correlations are compared with data. Including the additional medium effects results in the dashed curves. 
Figure 1

Figure 2
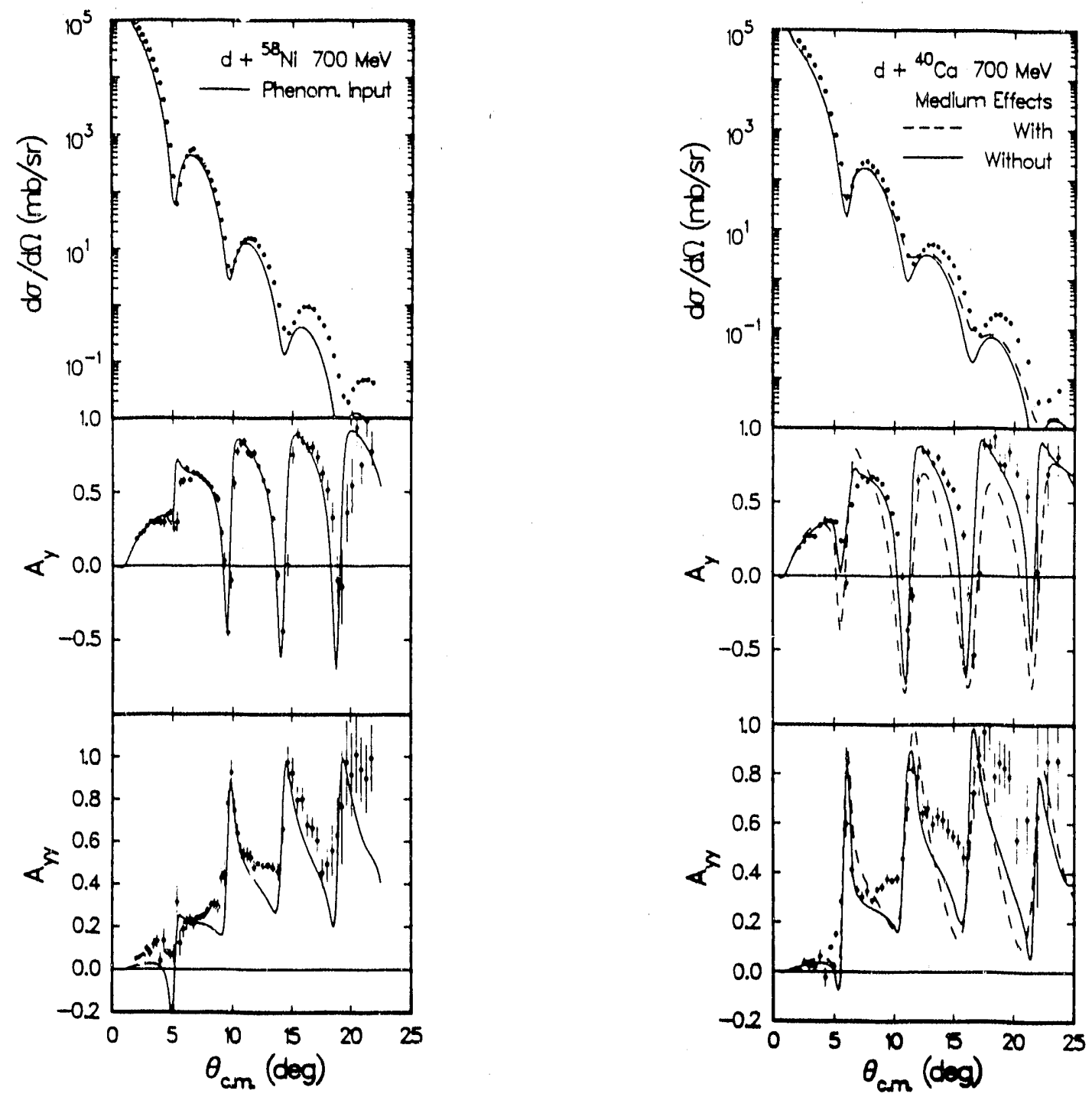


\title{
8.3 Coupled-Channels Dirac Equation Treatment of Large Angle $\vec{p}+{ }^{40} \mathrm{Ca}$ at $500 \mathrm{MeV}$
}

\author{
(L. Ray and G. W. Hoffmann; Ohio State; IBM; Saclay; Alberta)
}

As part of our study of relativistic effects in proton-nucleus scattering we have conducted a series of coupled-channels Dirac equation calculations for $\vec{p}+{ }^{40} \mathrm{Ca}$ elastic and inelastic scattering at $500 \mathrm{MeV}{ }^{1.2} \mathrm{~A}$ longstanding problem in intermediate energy proton-nucleus physics concerns the inability of microscopic, theoretical models, either relativistic or nonrelativistic, to qualitatively reproduce angular distributions beyond momentum transfers of $3 \mathrm{fm}^{-1}$. It was originally suggested ${ }^{3}$ that coupling to strong, collective states was responsible for the disagreement with data. However, the necessity of including relativistic dynamics and deformed spinorbit coupling hindered the success of coupled-channels calculations for several years.

We have now overcome these difficulties and have performed calculations using relativistic and nonrelativistic impulse approximation models for the optical potential and a collective model for inelastic transitions. Our calculations include coupling to strong collective states, rclativistic dynamics, and deformed spin-orbit coupling and were made with the codes ECIS87 and CENITH. ${ }^{5}$

The effects of channel-coupling in the RIA - Dirac equation coupled-channels model for elastic scattering are shown in Figs. 1 and 2, where the solid (dashed) curves include (do not include) coupling to the first $3^{-}$state in ${ }^{40} \mathrm{Ca}$. With channelcoupling, the RIA - Dirac model is now able to reproduce the elastic scattering data ${ }^{6}$ through 10 orders of magnitude! In Figs. 3 and 4 RIA (solid curves) and NRIA (dashed curves) coupled-channels predictions for elastic scattering are compared with data. The nonrelativistic predictions are simple, first-order results (i.e., no medium corrections or correlations). The differences between the dashed and solid curves display the effects of relativistic dynamics. The corresponding relativistic (solid curves) and nonrelativistic (dashed curves) predictions for the first few collective excitations in ${ }^{40} \mathrm{Ca}$ are shown in Figs. 5 and 6 in comparison with data. $^{7}$ The superiority of the relativistic predictions is evident. Two publications ${ }^{1,2}$ have resulted from this work.

\section{References}

[1] S. Shim, B. C. Clark, S. Hama, E. D. Cooper, R. L. Mercer, L. Ray, and G. W. Hoffmann, Phys. Rev. C 38, 1968 (1988).

[2] S. Shim, B. C. Clark, E. D. Cooper, S. Hama, R. L. Mercer, L. Ray, and J. Raynal, Phys. Rev. C (1990), in press.

[3] R. D. Amado and D. A. Sparrow, Phys. Rev. C 29, 932 (1984). 
[4] J. Raynal, Phys. Lett. B 196, 7 (1987).

[5] R. L. Mercer, Phys. Rev. C 15, 1786 (1977).

[6] G. W. Hoffmann et al., Phys. Rev. C 37, 1307 (1988); A. Rahbar et al., Phys. Rev. Lett. 47, 1811 (1981).

[7] M. L. Barlett, G. W. Hoffmann and L. Ray, Phys. Rev. C 35, 2185 (1987).

\section{Figure Captions}

Figure 1. The solid curve is the result of a coupled-channels RIA-Dirac equation model calculation for $500 \mathrm{MeV} \vec{p}+{ }^{40} \mathrm{Ca}$ elastic scattering. The dashed curve results when coupling to the $3^{-}$state is omitted.

Figure 2. Same as Fig. 1 except the analyzing power and spin rotation function are shown.

Figure 3. The RIA (solid curves) and NRIA (dashed curves) coupled-channels results for $500 \mathrm{MeV} \vec{p}+{ }^{40} \mathrm{Ca}$ are compared with cross section data.

Figure 4. Same as Fig. 3 except the analyzing power and spin rotation function.

Figure 5. The relativistic (solid) and nonrelativistic (dashed) coupled-channels results for the first few collective excitations in $500 \mathrm{MeV} \vec{p}+{ }^{40} \mathrm{Ca}$ are compared with cross section data.

Figure 6. Same as Fig. 5 except analyzing power. 

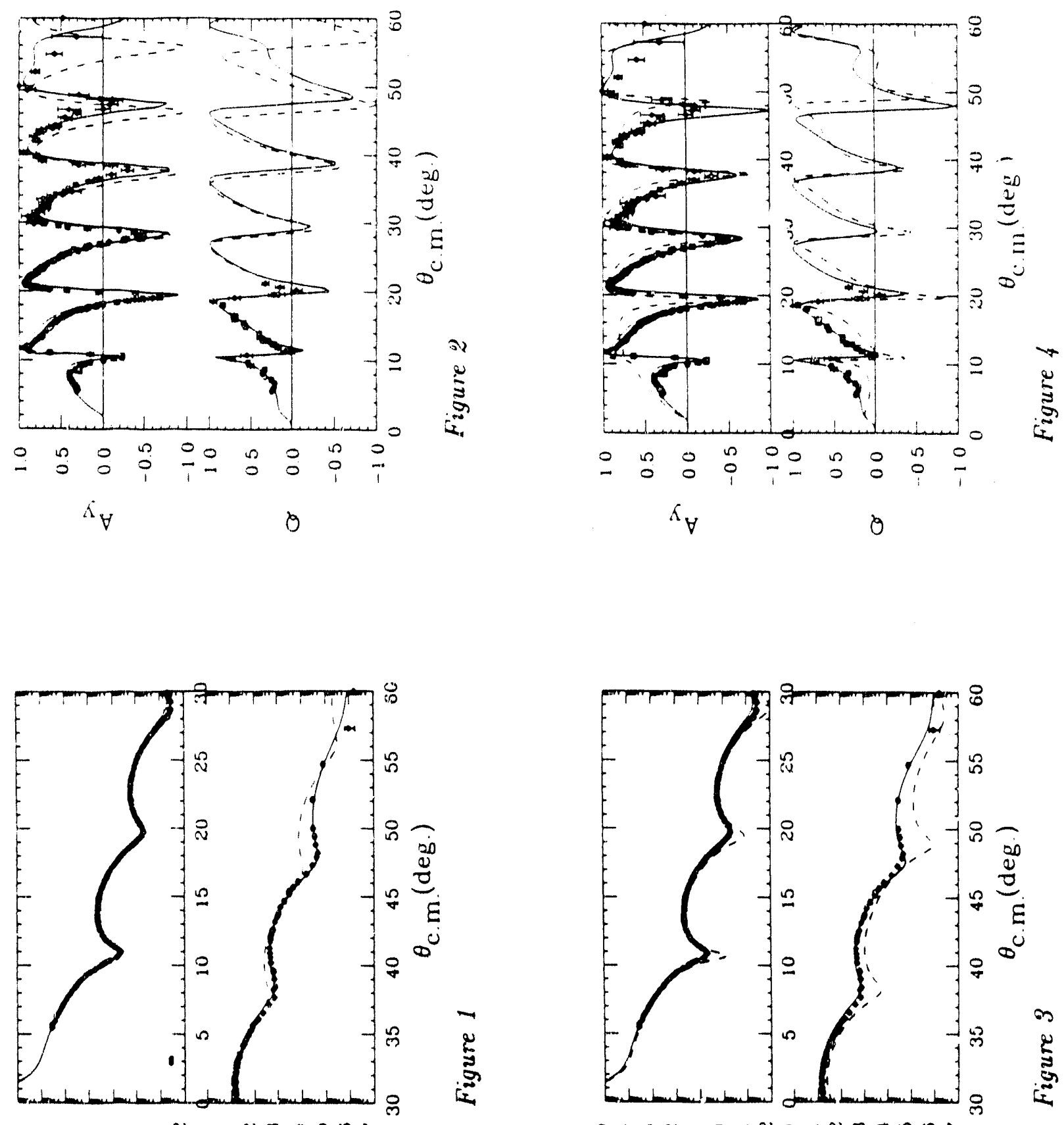

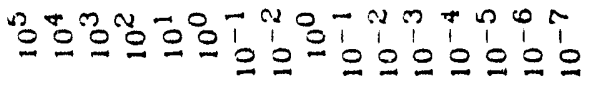

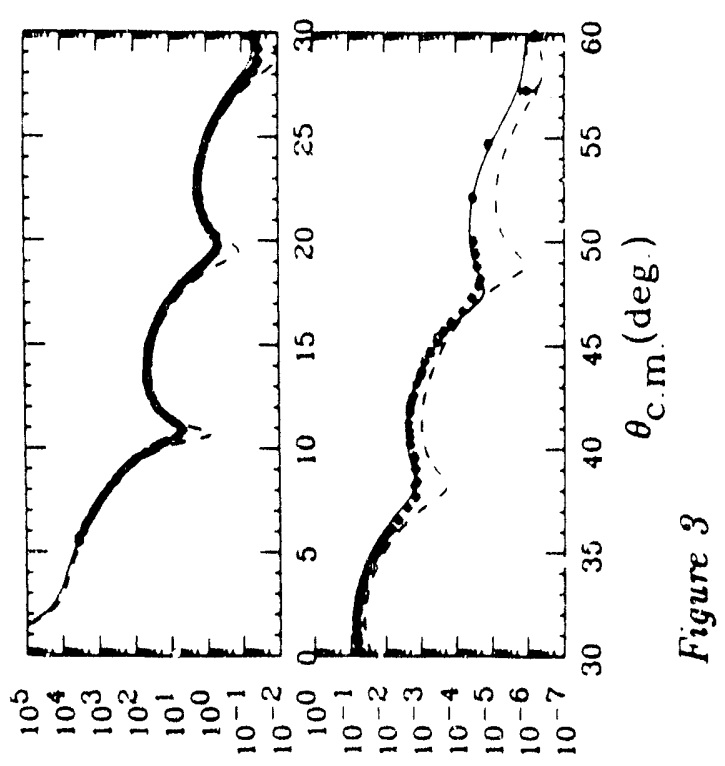

(ss/qu) up/op

(ss/qu) UP/OP 

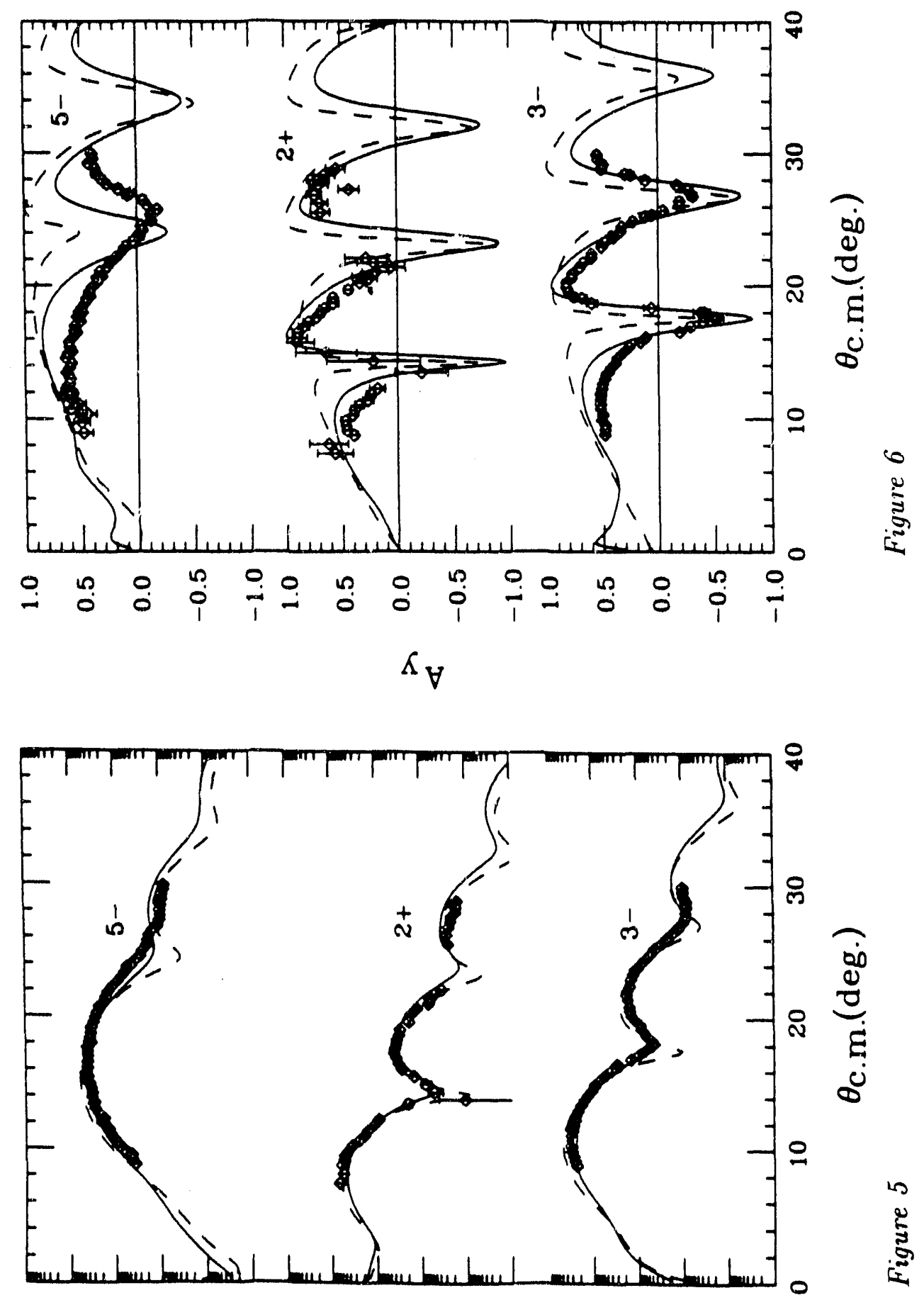

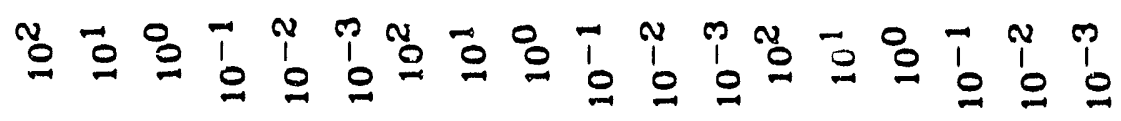

(Is/qu) Up/op 


\subsection{Antisymmetrization Correction for Nucleon-Nucleus Elastic Scattering}

(L. Ray)

hs part of our gener.' 'study of the nucleon-nucleus optical potential and the effective nucleon-nucleon interaction in intermediate energy proton-nucleus scattering we have evaluated the leading antisymmetrization correction which results in full, $(A+1)$-body antisymmetrized theories of the optical potential. Traditional multiple scattering theories, ${ }^{1}$ which are most often applied in nucleon-nucleus scattering calculations, do not account for the identical nature of the projectile and the nucleon constituents of the target nucleus except at the two-body level according to the prescription of Takeda and Watson (TW). ${ }^{2}$ In this work ${ }^{3}$ a fully antisymmetrized nucleon-nucleus optical potential formalism ${ }^{4}$ was used to evaluate the leading antisymmetrization correction to the TW prescription. The new term is a three-body exchange potential which is linear in the NN effective interaction operator. From Ref. 3 this term is

$$
P U_{A} P \simeq-\sum_{i \neq j} P T_{i} E_{0 j} P,
$$

where $P$ projects the unsymmetrized elastic channel, $T_{i}$ is the projectile $-i^{\text {th }}$. target-nucleon $t$ matrix, and $E_{0 j}$ exchanges the projectile nucleon $(0)$ and target nucleon $(j)$. In applications of the unsymmetrized theory, the proper symmetry between target nucleons $(i)$ and $(j)$ is included in the antisymmetrized target wave function while that involving particles $(0)$ and $(i)$ is correctly treated by way of the $\mathrm{NN}$ effective interaction. The new term correcis the unsymmetrized optical potential by including the exchange of particies $(0)$ and $(j)$ while particles $(0)$ and (i) interact.

Calculations were performed for $p+{ }^{16} \mathrm{O}$ and ${ }^{40} \mathrm{Ca}$ over a range of incident laboratory kinetic energies. Selected results are summarized in Table I which lists for each case the volume per nucleon of the empirical real, spin-independent protun + nucleus optical potential $\left(U_{\text {cent }}\right)$, that of the local equivalent approximation to the antisymmetrization correction $\left(U_{A, \text { Local }}^{00}\right)$, and the absolute value of the ratio of the two in percent. The correction diminishes rapidly with increasing energy, however, below $30 \mathrm{MeV}$ the effect is several percent. Therefore for incident energies of several tens to hundreds of $\mathrm{MeV}$ the $\mathrm{TW}$ prescription is completely adequate. The dominant effect of the Pauli exclusion principle in nucleon-nucleus scattering continues to be that due to the blocking effect on the $\mathrm{NN}$ intermediate scattering states. 


\section{References}

[1] K. M. Watson, Phys. Rev. 89, 575 (1953); A. K. Kerman, H. McManus, and R. M. Thaler, Ann. Phys. (N. Y.) 8, 551 (1959).

[2] G. Takeda and K. M. Watson, Phys. Rev. 97, 1336 (1955).

[3] L. Ray, Phys. Rev. C 39, 1170 (1989).

[4] A. Picklesimer and R. M. Thaler, Phys. Rev. C 23, 42 (1981).

TABLE I. Optical potential volumes.

\begin{tabular}{lccc}
\hline \multicolumn{1}{c}{ Reaction } & $\begin{array}{c}R E\left[\left\langle U_{\text {cent }}\right\rangle\right] / A \\
\left(\mathrm{MeV} \mathrm{fm}^{3}\right)\end{array}$ & $\begin{array}{c}R E\left[\left\langle U_{\text {A, local }}^{00}\right)\right] / A \\
\left(\mathrm{MeV} \mathrm{fm}^{3}\right)\end{array}$ & $\begin{array}{c}\text { Absolute value } \\
\text { of ratio }(\%)\end{array}$ \\
\hline$p+{ }^{16} \mathrm{O}, 20.8 \mathrm{MeV}$ & -483.4 & 11.8 & 2.4 \\
$p+{ }^{16} \mathrm{O}, 30.4 \mathrm{MeV}$ & -449.2 & 4.7 & 1.05 \\
$p+{ }^{16} \mathrm{O}, 39.7 \mathrm{MeV}$ & -454.5 & 0.027 & 0.006 \\
$p+{ }^{16} \mathrm{O}, 49.5 \mathrm{MeV}$ & -419.7 & -0.29 & 0.07 \\
$p+{ }^{+0} \mathrm{Ca}, 9.86 \mathrm{MeV}$ & & & 3.9 \\
$p+{ }^{40} \mathrm{Ca}, 20.6 \mathrm{MeV}$ & -497.5 & 19.6 & 2.0 \\
$p+{ }^{+0} \mathrm{Ca}, 30.3 \mathrm{MeV}$ & -388.1 & 7.65 & 0.39 \\
$p+{ }^{+0} \mathrm{Ca}, 40 \mathrm{MeV}$ & -402.3 & -1.56 & 0.54 \\
$p+{ }^{40} \mathrm{Ca}, 49 \mathrm{MeV}$ & -392.5 & -2.12 & 0.31 \\
$p+{ }^{+0} \mathrm{Ca}, 76 \mathrm{MeV}$ & -302.8 & -0.94 & 0.007 \\
\hline \hline
\end{tabular}




\title{
8.5 Spin-One Kemmer-Duffin-Petiau Equation and Inter- mediate-Energy Deuteron-Nucleus Scattering
}

\author{
(L. Ray; LANL; OSU; IBM)
}

The success of relativistic approaches in intermediate energy proton-nucleus ( $p A)$ scattering encourages the application of relativistic equations to other probes of nuclei, such as the deuteron. We used the 10 dimensional, spin-one representation of the Kemmer-Duffin-Petiau (KDP) equation ${ }^{1}$ as the basis for a relativistic treatment of deuteron-nucleus scattering. The KDP formalism yields a first-orcier, multi-component relativistic equation for spin-1 particles given by

$$
\left(i \beta^{\mu} \partial_{\mu}-m-U\right) \psi=0,
$$

where $\beta^{\mu}$ represent a set of $10 \times 10$ matrices. ${ }^{1}$ We chose the KDP potential, $U$, to be a sum of scalar and vector terms, each of which was obtained by folding the Dirac phenomenological scalar and vector optical potentials for $p \mathrm{~A}$ scattering with the deuteron wave function. The KDP equation was manipulated into Schrödinger-like form resulting in effective central, spin-orbit, and tensor potentials.

In Fig. 1 the elastic scattering observables calculated with the KDP model are compared with $400 \mathrm{MeV} d+{ }^{58} \mathrm{Ni}$ differential cross section and vector and tensor analyzing power data. ${ }^{2}$ The predictions qualitatively describe the data. In Fig. 2, KDP predictions (solid curves) and the results of a Schrödinger equation-Watanabe model calculation ${ }^{3}$ (dashed curves) are compared with data ${ }^{2}$ for $700 \mathrm{MeV} d+{ }^{40} \mathrm{Ca}$ elastic scattering. Both calculations use the same Dirac phenomenological scalar and vector optical potential obtained by fitting $p+{ }^{40} \mathrm{Ca}$ elastic scattering data at $362 \mathrm{MeV}$. For the nonrelativistic calculations the Schrödinger equivalent central and spin-orbit potentials corresponding to the Dirac phenomenology for $p+{ }^{40} \mathrm{Ca}$ were folded with the deuteron wave function to produce the $d+{ }^{40} \mathrm{Ca}$ optical potential for use in the Schrödinger equation. Comparison of these two sets of calculations provides a concise delineation of the differences between the relativistic KDP and nonrelativistic Schrödinger equation approaches to deuteron-nucleus scattering. We find that the KDP method provides a reasonable starting point for describing such data.

\section{References}

[1] R. E. Kozack, B. C. Clark, S. Hama, V. K. Mishra, G. Käibermann, R. L. Mercer, and L. Ray, Phys. Rev. C 37, 2898 (1988); R. E. Kozack, B. C. Clark, S. Hama, V. K. Mishra, R. L. Mercer, and L. Ray, Phys. Rev. C 40, 2181 (1989).

[2] N. Van Sen et al., Phys. Lett. B 156, 185 (1985). 
[3] L. Ray, Phys. Rev. C 38, 2743 (1988).

\section{Figure Captions}

Figure 1. Elastic scattering observables calculated with the KDP mocel are compared with data for $400 \mathrm{MeV} d+{ }^{58} \mathrm{Ni}$.

Figure 2. KDP predictions (solid) and Schrödinger equation - Watanabe model results (dashed) are compared with data for $700 \mathrm{MeV} d+{ }^{40} \mathrm{Ca}$ elastic scattering.
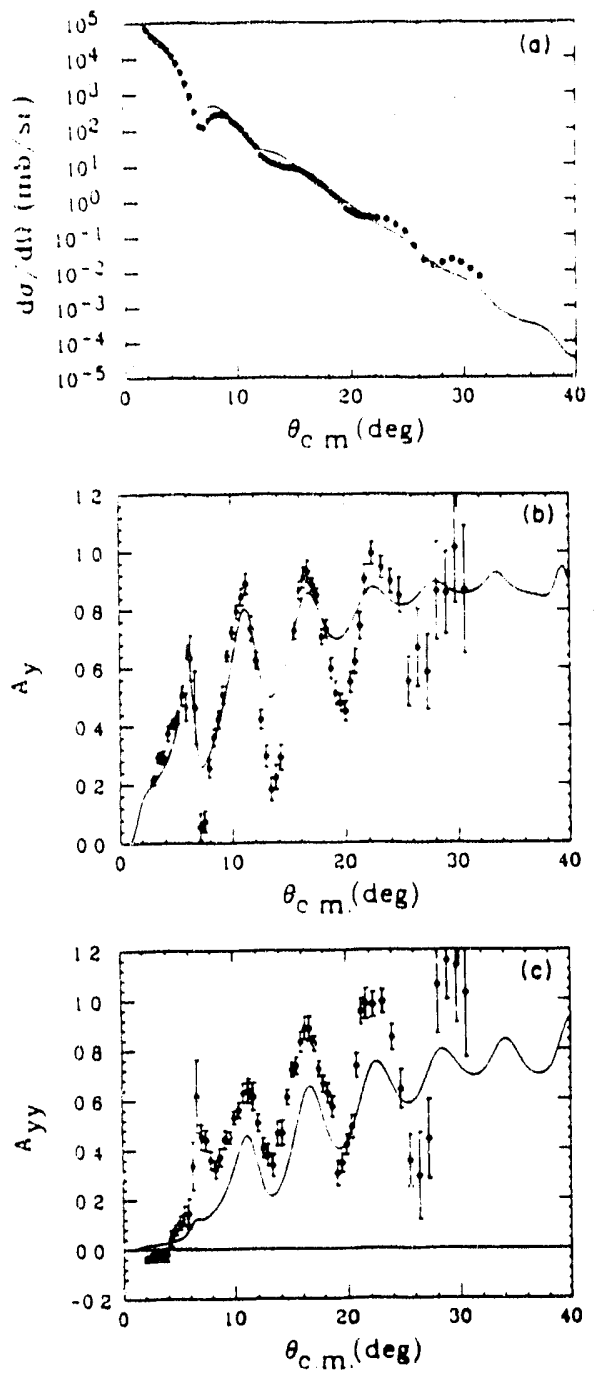

Figure 1
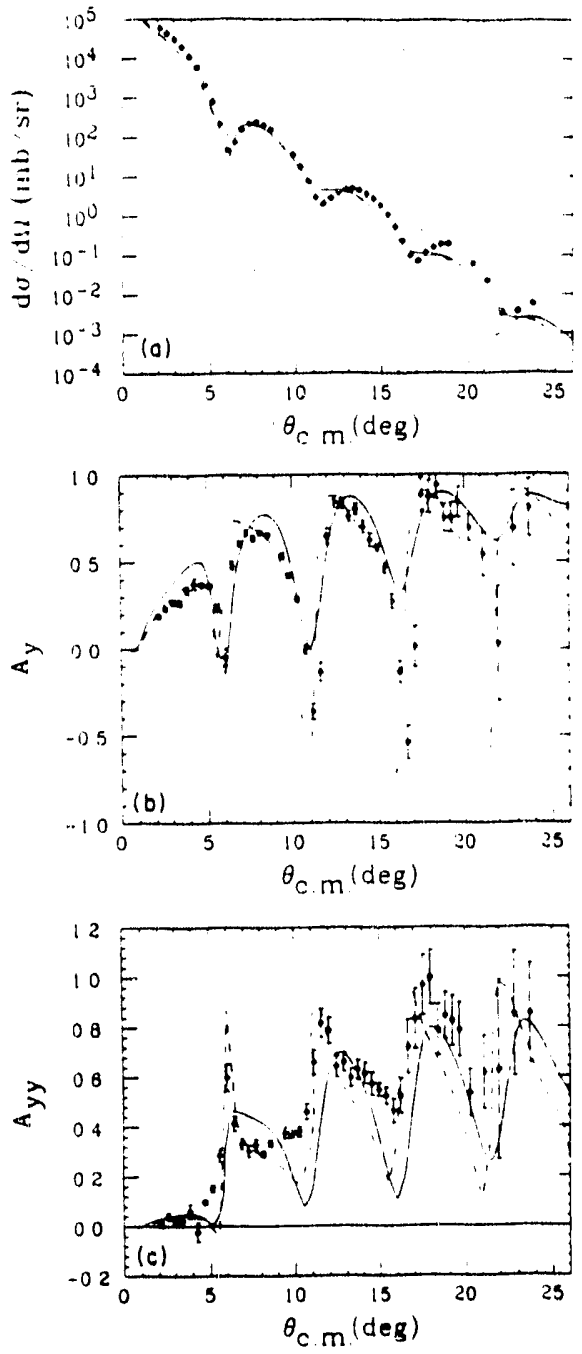

Figure 2 


\subsection{Effective Interactions for Nucleon-Nucleus Scattering Above $300 \mathrm{MeV}$}

(L. Ray)

Recently a major calculation was completed of the $\mathrm{NN}$ effective interaction in the nuclear medium at intermediate energies. ${ }^{1}$ The principal motivation for this work was the fact that all previous nonrelativistic calculations of nucleon-nucleus scattering at energies above $400 \mathrm{MeV}$ relied on the impulse approximation (IA); such models fail to quantitatively describe data, particularly the spin observables. Medium effects play an important role in describing nucleon-nucleus scattering data at lower energies and it is possible that such corrections might be significant at higher energies also. Furthermore the success of the relativistic Dirac equation models and the contrasting failure of the nonrelativistic IA predictions have brought into question the value of a nonrelativistic description of nuclei. Before such conclusions can be drawn the systematic corrections in both theories must be studied in more detail.

The present work involves a calculation of Pauli blocking and intermediate binding energy corrections to the nonrelativistic $N N$ effective interaction above $300 \mathrm{MeV}$. The nonrelativistic NN coupled-channels isobar model of Ref. 2 was used. This model is based on a meson exchange interaction together with nucleon-isobar $\left[\Delta(1232 \mathrm{MeV})\right.$ and $\left.N^{*}(1440 \mathrm{MeV})\right]$ coupling and short-range phenomenology. It successfully describes the $J \leq 6, T=0$ and $1 \mathrm{NN}$ scattering states from 0 to $1000 \mathrm{MeV}^{3}$

The nonrelativistic NN effective interaction of the Watson multiple scattering theory ${ }^{4}$ was approximately evaluated. It is given in $t$ matrix operator form by

$$
t^{W}=v+v G Q t^{W}
$$

where $v$ represents the $N N$ interaction (unmodified in the nuclear medium), Q projects all antisymmetric states of the target except the ground state, and the propagator, $\mathrm{G}$, is given by

$$
G=\left(E-H_{0}-H_{A}+i \epsilon\right)^{-1} .
$$

In the energy denominator $H_{0}$ is the kinetic energy operator and $H_{A}$ is the nuclear Hamiltonian.

Exact evaluation of $t^{W}$ represents a very formidable task. In this work the projected propagator $G Q$ for finite nuclei was replaced by that corresponding to two interacting nucleons in infinite nuclear matter with Fermi momentum $k_{F}$ where $N N, N \Delta$, and $N^{*}$ channels were included. Operator $Q$ becomes the usual Pauli blocking factor. The energy denominator in this approximation contains an intermediate binding potential $U$ which acts on only the target nucleon. This potential was assumed to have the velocity dependent form, $U\left(k^{2}\right)=A+B k^{2}$ where the parameters $A$ and $B$ were determined from optical model phenomenology. 
The free NN $t$ matrix is given by

$$
t=v+v g t
$$

where

$$
g=\left(E_{0}-H_{0}+i \epsilon\right)^{-1} .
$$

The free $\mathrm{NN} t$ matrix and that of the Watson theory are related by

$$
t^{W}=t+t(G Q-g) t^{W} .
$$

In these calculations the $\mathrm{NN}$ model was used to evaluate the second term on the right-hand-side of this expression. The evaluation of $t^{W}$ was facilitated by introducing a wave function $\psi$ defined by

$$
t^{W} \phi=v \psi
$$

where $\phi$ is a NN plane wave. The wave function $\psi$ was obtained by solving the integral equation

$$
\psi=\phi+G Q v \psi
$$

in coordinate space using standard partial wave expansions. The final, estimated density dependent on-momentum-shell $t$ matrix was obtained from the definition

$$
\left\langle t^{W}\left(k_{F}\right)\right\rangle_{\text {Final }} \equiv\langle t\rangle_{\text {Arndt }}+\left\langle t^{W}\left(k_{F}\right)-t^{W}\left(k_{F}=0\right)\right\rangle_{\text {Model }},
$$

where $\langle t\rangle_{\text {Arndt }}$ denotes the free $\mathrm{NN} t$ matrix obtained from the Arndt phase shift analysis. ${ }^{3}$

The proton-nucleus optical potentials were evaluated by folding the density dependent interactions with the nuclear density, using the local density approximation (LDA). The proton densities were obtained from electron scattering and the neutron densities from Hartree-Fock-Bogoliubov models. ${ }^{5}$ Second-order optical potential terms were also included arising from Pauli, short-range repulsion and center-of-mass correlations. ${ }^{6}$ Calculations were performed for $p+{ }^{16} \mathrm{O},{ }^{40} \mathrm{Ca}$, and ${ }^{208} \mathrm{~Pb}$ at proton incident energies of $300,320,400,500,613,650$, and $800 \mathrm{MeV}$. Differential cross sections, analyzing powers and spin rotations were computed and compared with available data.

The results for $p+{ }^{40} \mathrm{Ca}$ are shown in Figs. 1-3 where calculations with (without) density dependence are shown by the solid (dashed) curves. Medium effects tend to fill-in the minima in the differential cross sections, producing generally better agreement with the data. The structure in the analyzing powers is better reproduced. Problems remain in describing the forward angle $A_{y}$ data, but much of the original discrepancy in the IA predictions has been removed. Substantial improvement in the spin rotation predictions results when medium correcrions are included. These results are consistent with the medium effect calculations of von Geramb ${ }^{7}$ at the lower energies of 300 and $400 \mathrm{MeV}$. At higher energies further 
improvement results when electromagnetic spin-orbit effects are included. ${ }^{8}$ Similar results for ${ }^{16} \mathrm{O}$ and ${ }^{208} \mathrm{~Pb}$ are shown in Ref. 1 .

It is also important to evaluate the impact of medium corrections on the determination of nuclear structure which has been conducted over the last 10 years using the high quality, $800 \mathrm{MeV}$ elastic scattering data from the HRS at LAMPF. One such study involves fitting the cross section data for $p+{ }^{40} \mathrm{Ca}$ over a range of energies by varying the neutron density distribution. Fig. 4 shows the resulting neutron - proton root-mean-square radius difference (where $\Delta r_{n p} \equiv\left\langle r_{n}^{2}\right\rangle^{\frac{1}{2}}-\left\langle r_{p}^{2}\right\rangle^{\frac{1}{2}}$ ) in ${ }^{40} \mathrm{Ca}$ as a function of energy where medium corrections were included (solid dots) and where they were not included (crosses). The dashed line in the figure indicates the expected value. Medium corrections improve the agreement, however some unwanted energy dependence remains and the overall values of the extracted radii are still too small by about $0.2-0.3 \mathrm{fm}$, indicating that some dynamics is still missing in the model. Extraction of neutron isotopic density differences from nonrelativistic impulse approximation analyses of the $800 \mathrm{MeV}_{\text {data }}{ }^{6,9}$ was, however, shown to be unaffected by medium corrections.

This work shows that medium corrections to the intermediate proton-nucleus optical potential continue to be significant up to $800 \mathrm{MeV}$. Pauli blocking effects diminish quickly above energies of a few hundred $\mathrm{MeV}$ as expected. However, binding potential effects persist to higher energies. This work makes it clear that new physics issues associated with relativistic dynamics, possible changes in the bound nucleon form factors, variation in meson masses in the nuclear medium, etc., can only be reasonably studied when the physics included in conventional NR multiple scattering theory has been fully exploited in "he numerical applications.

\section{References}

[1] L. Ray, Phys. Rev. C 41, 2816 (1990).

[2] L. Ray, Phys. Rev. C 35, 1072 (1987).

[3] R. A. Arndt et al., Phys. Rev. D 28, 97 (1983).

[4] K. M. Watson, Phys. Rev. 89, 575 (1953).

[5] J. Dechargé and D. Gogny, Phys. Rev. C 21, 1568 (1980).

[6] L. Ray, Phys. Rev. C 19, 1855 (1979).

[7] L. Rikus, K. Nakano and H. V. von Geramb, Nucl. Phys. A414, 413 (1984).

[8] W. R. Coker and L. Ray, Phys. Rev. C 42, 659 (1990).

[9] L. Ray et al., Phys. Rev. C 23, 828 (1981). 


\section{Figure Captions}

Figures 1-3. Results of calculations for $p+{ }^{40} \mathrm{Ca}$ elastic scattering from 300$800 \mathrm{MeV}$. The solid curves show the results using density dependence and the dashed curves are the results without density dependence.

Figure 4. The deduced neutron - proton rms radius difference in ${ }^{40} \mathrm{Ca}$ as a function of energy. The solid dots indicate the results using the density dependent interaction; the crosses are the results without density dependence.

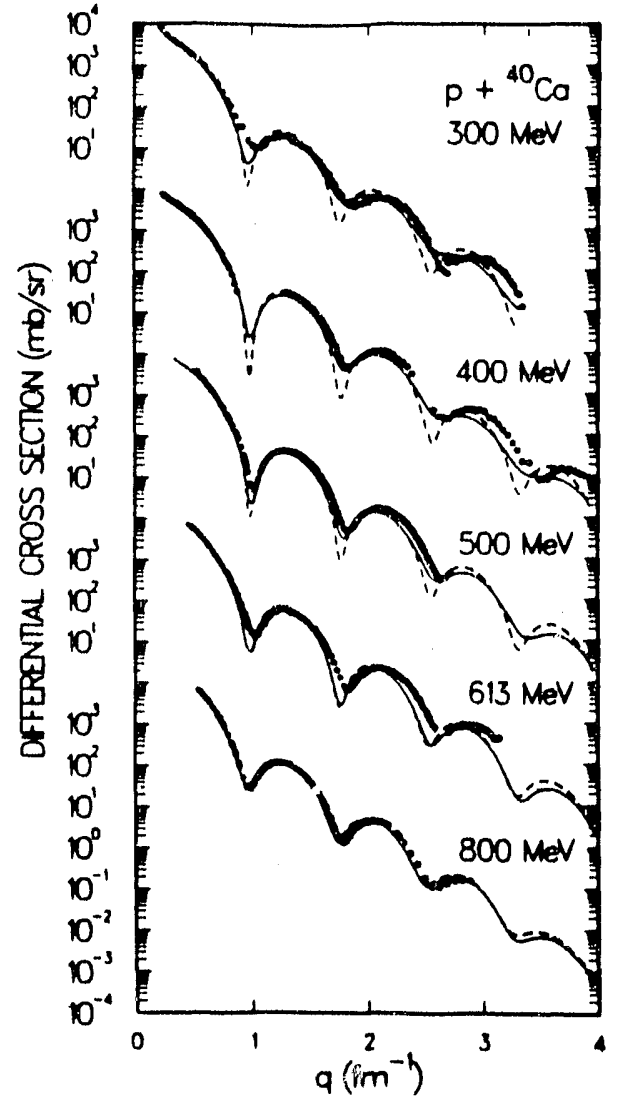

Figure 1

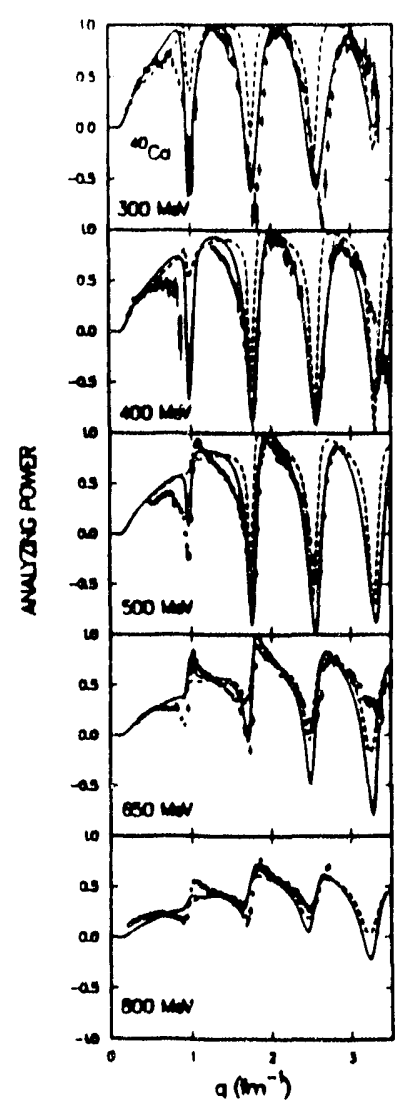

Figure 2 
Figure 3

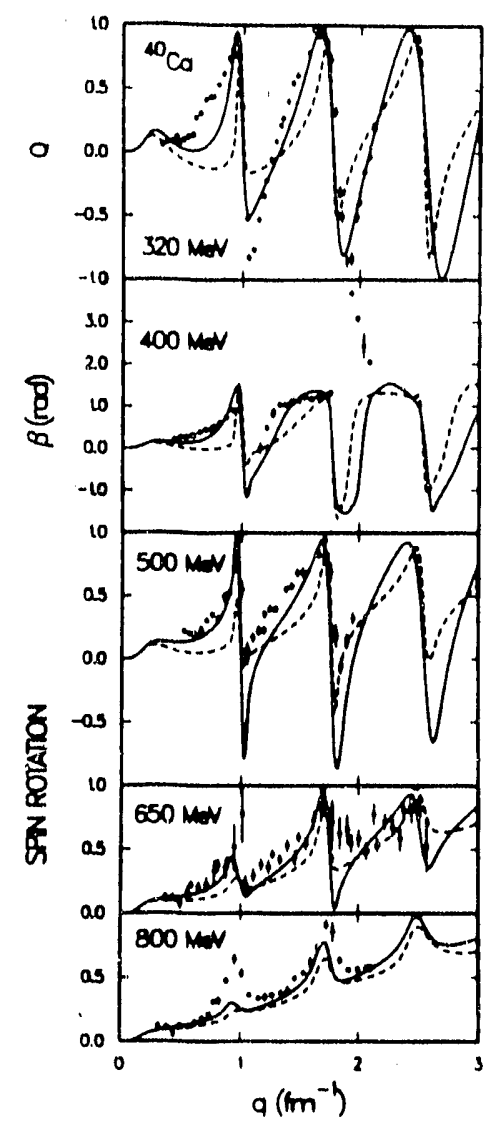

Figure 4

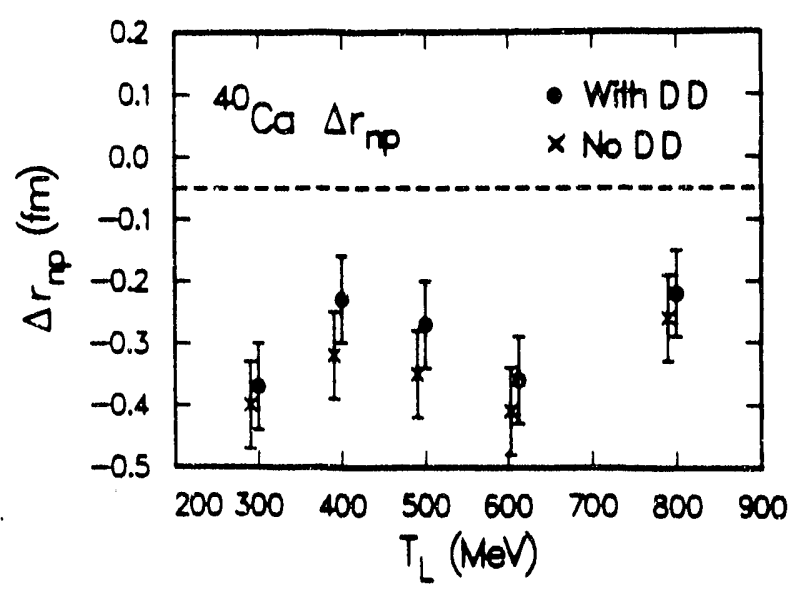




\subsection{Analyzing Powers for $\vec{p}+{ }^{208} \mathrm{~Pb}$ at Large Momentum Transfers}

(L. Ray; UCLA; LANL; Minnesota)

Analysis of large angle $\left(22^{\circ}-37^{\circ}\right.$ c.m. $)$ analyzing power data for $800 \mathrm{MeV} \vec{p}+{ }^{208} \mathrm{~Pb}$ elastic scattering and inelastic excitation to the $3^{-}, 2.61 \mathrm{MeV}$ state were carried out. ${ }^{1}$ The new measurements extend the momentum transfer range (to $4.7 \mathrm{fm}^{-1}$ ) and improve the statistical quality of previous data for this target. ${ }^{2}$ The data were taken with the HRS at LAMPF and are consistent, within statistics, with previous measurements. ${ }^{2}$ Analyses of large-angle differential cross-section and analyzing power data can in principle provide new information about off-shell, correlation, and multistep effects.

The new data and theoretical predictions are shown in the figure along with the previous data (solid squares) of Ref. 2. The dashed and solid curves for elastic scattering represent theoretical predictions that use nonrelativistic impulse approximation (NRIA) - Schrödinger equation and relativistic impulse approximation (RIA) - Dirac equation models, respectively. Both calculations use Arndt SP87 NN amplitudes and a proton density determined by electron scattering. Neutron densities were varied in order to fit the forward angle differential cross-section data. Scalar densities for the relativistic model were obtained from relativistic mean field models. ${ }^{3}$ The overall structure of the elastic analyzing power data is better reproduced by the RIA model.

The predictions for inelastic scattering were made by using the standard DWBA collective model with deformed spin-orbit coupling. For the RIA prediction the Schrödinger equivalent potential of the Dirac optical potential provided the input for the DWBA calculations. The RIA based predictions are somewhat better.

\section{References}

[1] M. G. Moshi, C. A. Whitten, Jr., B. Aas, G. Igo, D. Lopiano, L. Ray, K. Jones, and M. Gazzaly, Phys. Rev. C 41, 1279 (1990).

[2] G. W. Hoffmann et al., Phys. Rev. C 24, 541 (1981).

[3] C. J. Horowitz and B. D. Serot, Nucl. Phys. A368, 503 (1981). 


\section{Figure Caption}

Figure 1. New analyzing power data for $800 \mathrm{MeV} \vec{p}+{ }^{208} \mathrm{~Pb}$. The solid curves are the relativistic model predictions, while the dashed curves are the nonrelativistic model predictions.
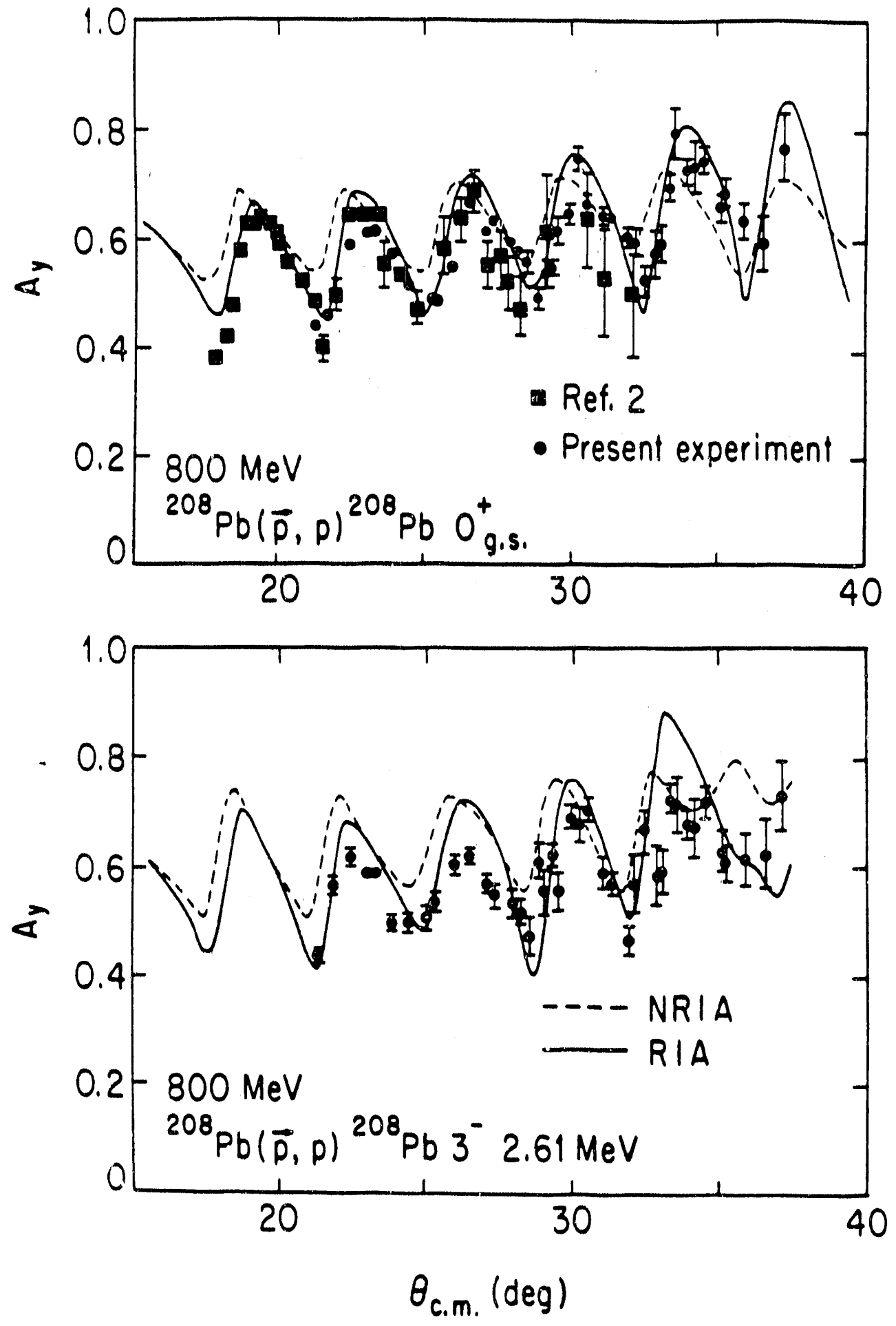


\subsection{Comparison of Dirac and Schrödinger Descriptions of Spin Observables for Proton-Nucleus Elastic Scatter- ing at 650 and $800 \mathrm{MeV}$}

(L. Ray and W. R. Coker (Univ. of Texas))

The significant improvement in the nonrelativistic (NR) Schrödinger equation description of proton-nucleus elastic scattering brought about by medium corrections to the effective NN interaction ${ }^{1}$ warrant further investigation of NR scattering models. At 650 and $800 \mathrm{MeV}$ electromagnetic effects due to the coupling between the projectile proton's magnetic moment and the current induced by the motion of the target nucleus electric charge (in the c.m.) qualitatively affect the forward angle spin observable predictions. ${ }^{2} \mathrm{NR}$ calculations have been made ${ }^{3}$ in which medium effects, correlations, and the electromagnetic spin-orbit (EMSO) potential were all included. These were compared with data and with predictions of the relativistic impulse approximatio i (RIA) - Dirac equation approach ${ }^{4}$ where the proton anomalous magnetic moment term was included.

The NR calculations including medium corrections and correlations are described in section 8.6 of this document and in Ref. 1. The electromagnetic spinorbit interaction adds a $1 / q$ dependent term to the single spin-flip amplitude, $c(q) \vec{\sigma}^{p} \cdot \hat{n}$, which leads to a long-range contribution to the proton-nucleus spinorbit optical potential which is of the form,

$$
U_{E M S O} / r^{3} \vec{\sigma} \cdot \vec{\ell},
$$

where $U_{E M S O}$ is a complex number. This long-range potential was handled in the calculations by ray of a Gaussian convergence factor procedure. ${ }^{3}$

The RIA calculations were carried out as in Ref. 4 except a corrected form for the nuclear tensor potential was used ${ }^{5}$ and the proton anomalous magnetic moment term was included. Both calculations used the SP89 NN phase shift solution of Arndt and the same proton and neutron (vector) densities determined from electron scattering analyses and Hartree-Fock-Bogoliubov theory, respectively. The scalar and tensor densities for the RIA calculations were taken from relativistic mean field theory.

Calculations were done for $p+{ }^{16} \mathrm{O},{ }^{40} \mathrm{Ca}$ and ${ }^{208} \mathrm{~Pb}$ at 650 and $800 \mathrm{MeV}$. The results for ${ }^{40} \mathrm{Ca}$ and ${ }^{208} \mathrm{~Pb}$ are shown in Figs. 1 and 2, respectively. The solid curves are the nonrelatvistic predictions, including the EMSO potential, medium corrections, and correlations. The dashed curves indicate the RIA predictions while the dashed-dotted curves represent NR predictions without the EMSO term, but including correlations and medium effects.

For both nuclei the EMSO potential has significant effects at forward angles (especially for ${ }^{208} \mathrm{~Pb}$ ) and, for the model discussed here, results in improved descriptions of the data. Overall, both the NR and RIA predictions are in good 
agreement with earh other and the data. The same is true for the ${ }^{16} \mathrm{O}$ cases. ${ }^{3} \mathrm{Ap}$ plication of the NR, density-dependent optical potential with the EMSO potential will be don for cases at lower energies.

\section{References}

[1] L. Ray, Phys. Rev. C 41, 2816 (1990).

[2] R. W. Fergerson et al., Phys. Rev. C 33, 239 (1986).

[3] W. R. Coker and L. Ray, Phys. Rev. C 42, 659 (1990).

[4] L. Ray and G. W. Hoffmann, Phys. Rev. C 31, 538 (1985).

[5] W. R. Coker and L. Ray, Phys. Rev. C (1990), in press.

\section{Figure Captions}

Figures 1-2. The solid curves are predictions from the nonrelativistic model, including EMSO potential, medium corrections and correlations. The dashed curves are the corresponding relativistic model predictions. The dashed-dotted curves are the nonrelativistic model results when the $\mathrm{EMSO}$ effect is omitted, but medium effects and correlations are included. 
Figure 1
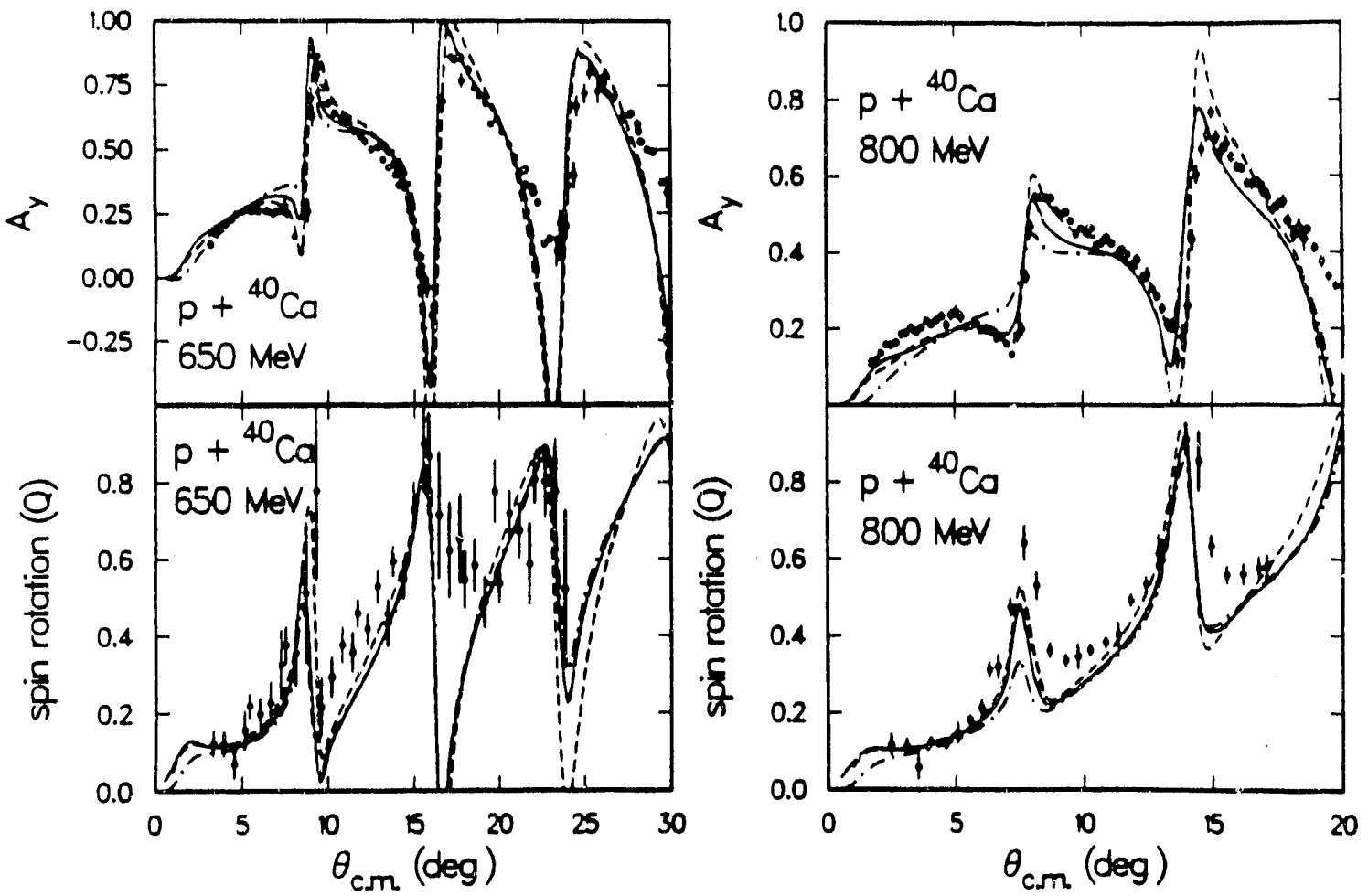

Figure 2
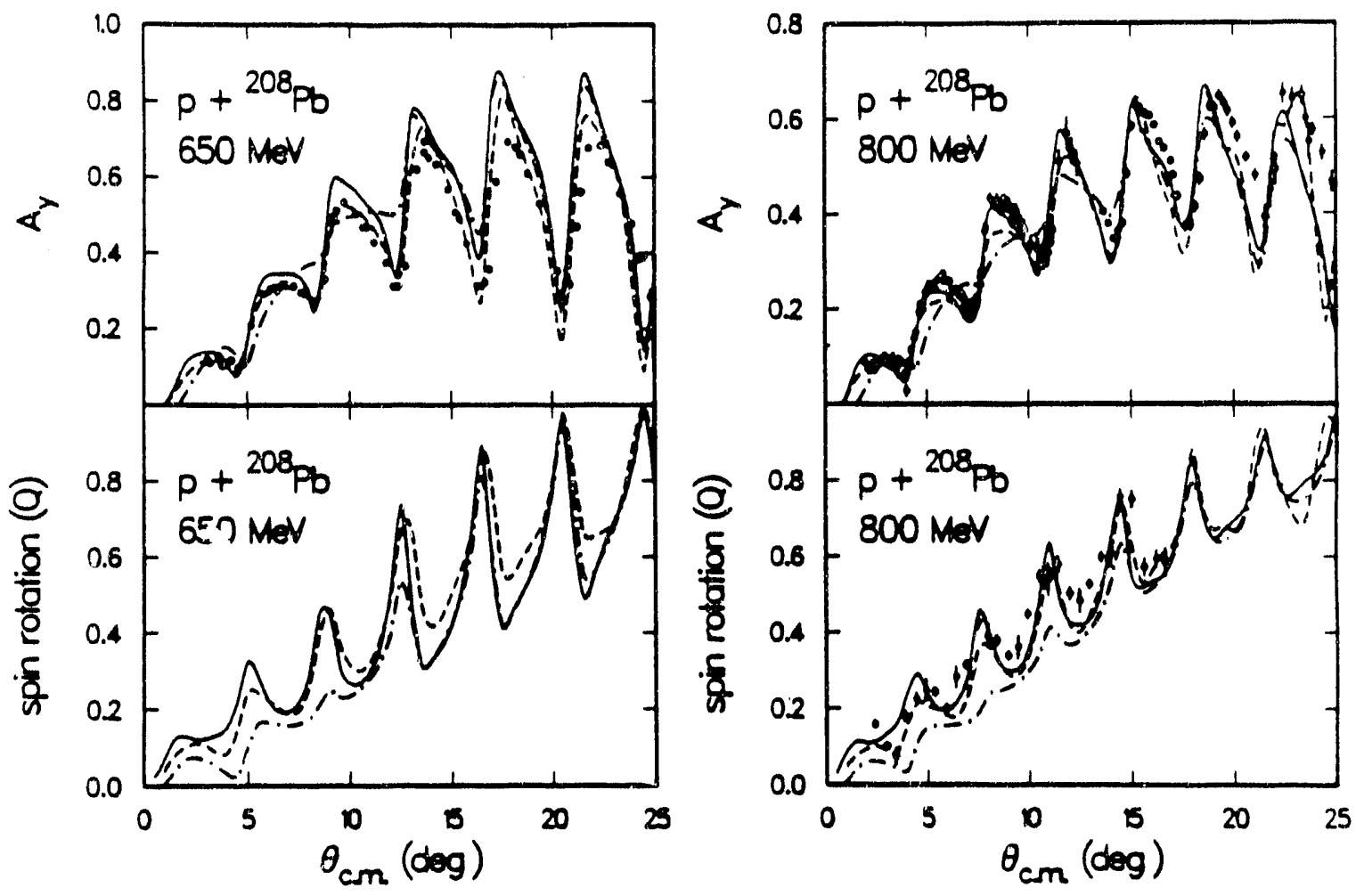


\subsection{Nonrelativistic Density-Dependent Calculations for $\vec{p}+{ }^{13} \overrightarrow{\mathrm{C}}$}

(L. Ray)

The nonrelativistic density-dependent effective interaction model developed by our group $^{1}$ has been used to predict the spin observables for polarized proton elastic scattering from polarized ${ }^{13} \mathrm{C}$. The calculations use a NR-DWBA formalism, similar to that developed in Ref. 2 for the relativistic $p+{ }^{13} \mathrm{C}$ calculation. The nonrelativistic DWBA matrix element for the valence nucleon contribution to the $p+{ }^{13} \mathrm{C}$ elastic scattering amplitude can be evaluated using four component forms for the distorted waves and bound state wave functions together with the five term, McNeil-Ray-Wallace ${ }^{3}$ form for the Lorentz invariant $N N$ interaction. This is true provided all lower components of wave functions are set to their nonrelativistic limits. ${ }^{2}$ The RIA-DWBA computer code (DIAMOND) can then be directly used for nonrelativistic density-dependent caiculations, when suitably modified for the density-dependent interaction.

Calculations for $500 \mathrm{MeV} \vec{p}+{ }^{13} \overrightarrow{\mathrm{C}}$ elastic scattering, $\hat{n}$-typ $\epsilon$ polarized target spin observables $A_{\text {ovon }}$ and $A_{\text {oonn }}$ are shown in Figs. 1 and 2. The solid curves indicate the NRIA-DWBA calculations also given in Ref. 4. The dashed curves represent the same NRIA-DWBA calculation except the newer, SP89 NN phase shift solution was used. The dashed-dotted curves are the nonrelativistic densitydependent prerjictions using the SP89 NN phase shift solution and the densitydependent ccrrections from Ref. 1 for each component of the NN force. Both the upgrade to more recent phase shift solutions (i.e., from SP82 to SP89) and density-dependence have only modest effects on the observables, compared to the differences between theory and data.

\section{References}

[1] L. Ray, Phys. Rev. C 41, 2816 (1990).

[2] J. Ray et al., Phys. Rev. C 37, 1169 (1988).

[3] J. A. McNeil, L. Ray and S. J. Wallace, Phys. Rev. C 27, 2123 (1983).

[4] G. W. Hoffmann et ril., submitted to Phys. Rev. Lett. (1990). 


\section{Figure Captions}

Figures 1-2. The solid curves are the NRIA-DWBA results of Ref. 4 for $500 \mathrm{MeV} \vec{p}+{ }^{13} \overrightarrow{\mathrm{C}}$ observables $\mathrm{A}_{\text {ooon }}$ and $\mathrm{A}_{\text {oonn }}$. The dashed curves resulted when newer SP89 NN amplitudes were used. The dashed-dotted curves result from using the SP89 amplitudes and density dependent corrections.

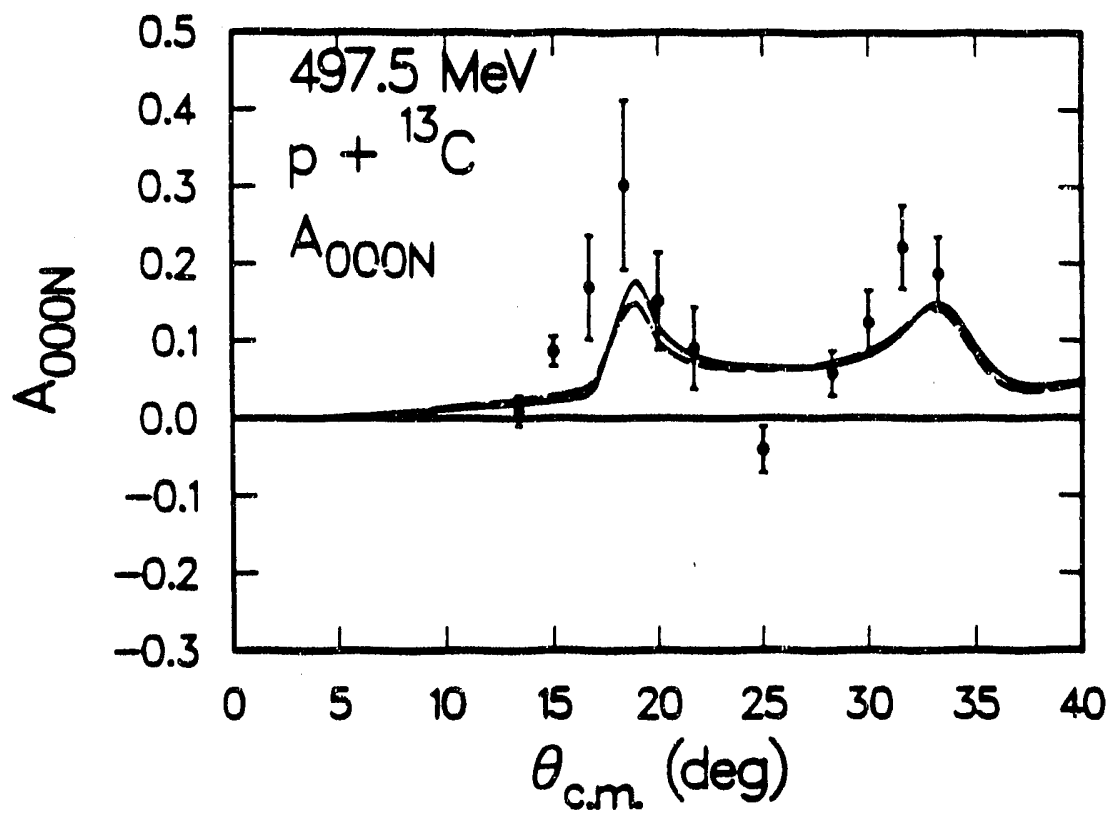

Figure 1

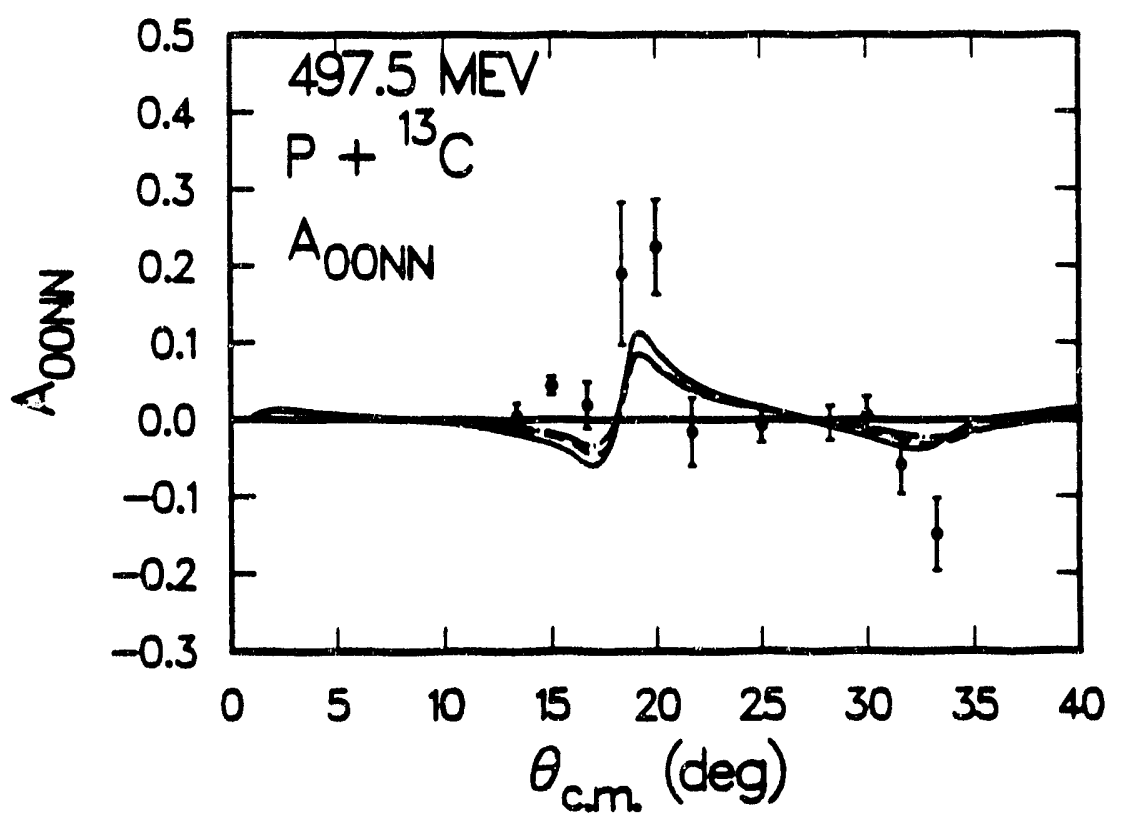

Figure 2 


\section{Publications (1988 - 1990)}

\subsection{Journal and Conference Proceedings in Print}

1. G. W. Hoffmann, M. L. Barlett, R. W. Fergerson, J. A. Marshall, J. A. McGill, E. C. Milner, L. Ray, and J. F. Amann, "Differential Cross Section and Analyzing Power Measurements for $p+p$ Elastic Scattering at 497.5 MeV," Phys. Rev. C 37, 397 (1988).

2. G. S. Blanpied, B. G. Ritchie, M. L. Barlett, R. W. Fergerson, J. A. McGill, and B. H. Wildenthal, "Elastic and Inelastic Scattering of $0.8 \mathrm{GeV}$ Protons from ${ }^{40}$ Ar," Phys. Rev. C 37, 1304 (1988).

3. L. Ray, G. W. Hoffmann, M. L. Barlett, J. D. Lumpe, B. C. Clark, S. Hama, and R. L. Mercer, "Relativistic Impulse Approximation Description of Polarized Proton Elastic Scattering from Yolarized ${ }^{13}$ C," Phys. Rev. C 371169 (1988).

4. G. S. Blanpied, B. G. Ritchie, M. L. Barlett, G. W. Hoffmann, J. A. McGill, E. C. Milner, K. W. Jones, S. K. Nanda, and R. de Swiniarski, "Elastic and Inelastic Scattering of $0.8 \mathrm{GeV}$ Polarized Protons from ${ }^{24} \mathrm{Mg}$ and ${ }^{26} \mathrm{Mg}$," Phys. Rev. C 37, 1987 (1988).

5. N. M. Hintz, D. Cook, M. Gazzaly, M. A. Franey, M. L. Barlett, G. W. Hoffmann, R. Fergerson, J. McGill, J. B. McClelland, and K. W. Jones, "Energy Dependence of Neutron-Proton Matrix Element Ratios Derived from 25-800 MeV Energy Proton Scattering," Phys. Rev. C 37, 692 (1988).

6. L. Ray, G. W. Hoffmann, M. L. Barlett, and N. Ottenstein, "Relativistic Effects in Forward Angle Proton-Nucleus Elastic Scattering," Phys. Rev. C 37, 224 (1988).

7. G. W. Hoffmann, M. L. Barlett, G. Pauletta, L. Ray, J. F. Amann, K. Jones, J. B. McClelland, R. W. Fergerson, M. M. Gazzaly, B. C. Clark, and R. L. Mercer, "Large Angle $\vec{p}+{ }^{40} \mathrm{Ca}$ Elastic Scattering at $497.5 \mathrm{MeV}$," Phys. Rev. C 37, 1307 (1988).

8. R. E. Kozack, B. C. Clark, S. Hama, V. K. Mishra, G. Kälbermann, R. L. Mercer, and L. Ray, "Relativistic Deuteron-Nucleus Scattering in the Kemmer-Duffin-Petiau Formalism," Phys. Rev. C 37, 2898 (1988).

9. N. Tanaka, M. M. Gazzaly, G. Pauletta, B. Aas, D. Adams, H. Fujisawa, S. Greene, H. Hasai, G. Igo, S. Isagawa, S. Ishimoto, K. Iwatani, K. Jones, D. Lopiano, A. Masaike, F. Nishiyama, Y. Ohashi, A. Okihana, S. Okumi, F. Sperisen, G. Weston, and C. Whitten, "Spin Dependence of $p p$ Inelasticities at Intermediate Energies," Phys. Rev. C 37, 2071 (1988). 
10. B. E. Bonner, J. A. Buchanan, D. C. Carey, J. M. Clement, R. N. Coleman, M. D. Corcoran, J. D. Cossairt, A. A. Derevshchikov, D. P. Grosnick, D. A. Hill, K. Imai, F. Lehar, A. de Lesquen, D. Lopiano, F. C. Luehring, J. W. Kruk, K. Kuroda, T. Maki, S. Makino, A. Masaike, Yu. A. Matulenko, A. P. Meshchanin, A. Michalowicz, H. E. Miettinen, D. H. Miller, K. Miyake, A. H. Mohammadzadeh, G. S. Mutchler, T. Nagamine, F. Nessi-Tadaldi, M. Nessi, S. B. Nurushev, C. Nguyen, Y. Ohashi, G. Pauletta, A. Penzo, G. C. Phillips, A. L. Read, J. B. Roberts, L. van Rossum, G. Salvato, P. Schiavon, T. Shima, V. L. Solovyanov, H. M. Spinka, R. W. Stanek, P. M. Stevenson, R. Takashima, F. Takeuchi, D. G. Underwood, A. N. Vasiliev, A. Villari, J. L. White, A. Yokosawa, T. Yoshida, A. Zanetti, and Q. Zhu, "Analyzing-Power Measurement in Inclusive $\pi^{0}$ Production at High $x_{F}$," Phys. Rev. Lett. 61, 1918 (1988).

11. G. Pauletta, M. Gazzaly, N. Tanaka, B. Aas, D. Adams, H. Fujisaw:, S. Greene, H. Hasai, G. Igo, S. Ishimoto, K. Jones, D. Lopiano, A. Masaike, K. Iwatani, Y. Ohashi, S. Okumi, L. Santi, F. Sperisen, and C. Whitten, " $A_{L L}$ at Small Momentum Transfers for the First Complete Determination of the Forward pp Scattering Amplitudes," Phys. Lett. B 211, 19 (1988).

12. S. Shim, B. C. Clark, S. Hama, E. D. Cooper, R. L. Mercer, L. Ray, and G. W. Hoffmann, "Dirac Coupled Channels Calculation for $p+{ }^{40} \mathrm{Ca}$ Inelastic Scattering Using the Relativistic Impulse Approximation," Phys. Rev. C 38, 1968 (1988).

13. R. Fergerson, J. McGill, C. Glashausser, K. Jones, S. Nanda, Sun Zuxun, M. Barlett, G. Hoffmann, J. Marshall, and J. McClelland, "Polarization Transfer for Inclusive Proton-Nucleus Inelastic Scattering at $800 \mathrm{MeV}$," Phys. Rev. C 38, 2193 (1988).

14. G. S. Blanpied, B. G. Ritchie, M. L. Barlett, R. W. Fergerson, G. W. Hoffmann, J. A. McGill, and B. H. Wildenthal, "Elastic and Inelastic Scattering of $0.8 \mathrm{GeV}$ Protons from ${ }^{20} \mathrm{Ne}$ and ${ }^{22} \mathrm{Ne}$," Phys. Rev. C 38, 2180 (1988).

15. L. Santi, M. Barlett, D. Ciskowski, R. Garfagnini, M. M. Gazzaly, G. W. Hoffmann, K. W. Jones, M. A. Nasser, G. Pauletta, C. Smith, N. Tanaka, and R. Whitney, "Evidence for Narrow Structure in the Analyzing Power of the ${ }^{3} \mathrm{He}(\vec{p}, d) \mathrm{X}$ Reaction," Phys. Rev. C 38, 2466 (1988).

16. G. S. Adams, B. Höistad, J. M. Laget, B. Aas, K. Jones, J. A. McGill, G. S. Blanpied, C. S. Mishra, W. K. Mize, G. Pignault, C. S. Whisnant, S. M. Whitlow, and G. Pauletta, "Analyzing Powers for the Reaction ${ }^{2} \mathrm{H}(\vec{p}, \gamma){ }^{3} \mathrm{He}$ at $800 \mathrm{MeV}, "$ Phys. Rev. C 37, 1341 (1988).

17. L. Ray and J. R. Shepard, "Spin-Flip Cross Sections for ${ }^{13} \mathrm{C}(\vec{p}, \vec{n}){ }^{13} \mathrm{~N}$ (g.s.) at $500 \mathrm{MeV}$," Phys. Rev. C 40, 237 (1989). 
18. L. Ray, "Relativistic Dynamics in Polarized Proton Scattering from Polarized ${ }^{13} \mathrm{C}$," in the proceedings of the Workshop on Relativistic Nuclear Many-Body Physics, Ohio State University, Columbus, Ohio, June 6 - 9, 1988, edited by B. C. Clark, R. J. Perry, and J. P. Vary, (World Scientific, Singapore, 1989), pg. 427 .

19. L. Ray, "Multiple Scattering Description of Internediate Energy DeuteronNucleus Elastic Scattering," Phys. Rev. C 38, 2743 (1988).

20. D. Hill. T. Kasprzyk, J. J. Jarmer, S. Penttilä, M. Krumpolc, G. W. Hoffmann, and M. Purcell, "Dynamic Polarization of ${ }^{19} \mathrm{~F}$ in a Flourinated Alcohol," Nuclear Instruments and Methods in Physics Research A277, 319 (1989).

21. G. S. Adams, B. Höistad, J. M. Laget, B. Aas, K. Jones, J. A. McGill, G. S. Blanpied, C. S. Mishra, G. Pignault, C. S. Whisnant, and G. Pauletta, "Analyzing Powers for $\vec{p}+d \rightarrow{ }^{3} \mathrm{He}+\pi^{0}$ at $800 \mathrm{MeV}$," Phys. Lett. B 219, 399 (1989).

22. L. Ray, "Antisymmetrization Correction for Nucleon-Nucleus Elastic Scattering," Phys. Rev. C 39, 1170 (1989).

23. R. E. Kozack, B. C. Clark, S. Hama, V. K. Mishra, G. Kälbermann, R. L. Mercer, and L. Ray, "The Kemmer-Duffin-Petiau Formalism and Intermediate Energy Deutron-Nucleus Scattering," in the proceedings of the Workshop on Relativistic Nuclear Many-Body Physics, Ohio State University, Columbus, Ohio, June 6 - 9, 1988, edited by B. C. Clark, R. J. Perry, and J. P. Vary, (World Scientific, Singapore, 1989), pg. 476.

24. J. Rapaport, P. W. Lisowski, J. L. Ullmann, R. C. Byrd, T. A. Carey, J. B. McClelland, L. J. Rybarcyk, T. N. Taddeucci, R. C. Haight, N. S. P. King, G. L. Morgan, D. A. Clark, D. E. Ciskowski, D. A. Lind, R. Smythe, C. D. Zafiratos, D. Prout, E. R. Sugarbaker, D. Marchlenski, W. P. Alford, and W. G. Love, "Fermi and Gamow-Teller Strength in $p$-Shell Nuclei from $(p, n)$ Reactions at 492 and $590 \mathrm{MeV}$," Phys. Rev. C 39, 1929 (1989).

25. M. M. Gazzaly, M. Barlett, R. Garfagnini, G. W. Hoffmann, K. Jones, M. A. Nasser, G. Pauletta, L. Santi, C. Smith, N. Tanaka, and R. Whitney, "Narrow Structure in the Analyzing Power of the ${ }^{3} \mathrm{He}(\vec{p}, d) \mathrm{X}$ Reaction at 0.8 $\mathrm{GeV}$," Proceedings of the 8th International Symposium on High Energy Spin Physics, Sept. 10-17, 1988, Minneapolis, Minnesota, American Institute of Physics Conf. Proc. No. 187, Particles and Fields Series 37, edited by Kenneth J. Heller, (AIP, New York, 1989), p. 641.

26. S. I. Penttilä, J. F. Amann, J. J. Jarmer, K. W. Jones, N. Tanaka, M. L. Barlett, G. W. Hoffmann, W. F. Kielhorn, G. Pauletta, and M. Purcell, 
"First Polarization Results from the LAMPF ${ }^{13} \overrightarrow{\mathrm{C}}$ Target," Proceedings of the 8th International Symposium on High Energy Spin Physics, Sept. 10-17, 1988, Minneapolis, Minnesota, American Institute of Physics Conf. Proc. No. 187, Particles and Fields Series 37, edited by Kenneth J. Heller, (AIP, New York, 1989), p. 1281.

27. D. Hill, T. Kasprzyk, J. J. Jarmer, S. Penttilä, M. Krumpolc, G. W. Hoffmann, and $M$ : Purcell, "Dynamic Polarization of ${ }^{19} \mathrm{~F}$ in a Flourinated Alcohol," Proceedings of the 8th International Symposium on High Energy Spin Physics, Sept. 10-17, 1988, Minneapolis, Minnesota, American Institute of Physics Conf. Proc. No. 187, Particles and Fields Series 37, edited by Kenneth J. Heller, (AIP, New York, 1989), p. 1268.

28. G. Pauletta, "Tests of a Coulomb-Nuclear Polarimeter," Proceedings of the 8th International Symposium on High Energy Spin Physics, Sept. 10-17, 1988, Minneapolis, Minnesota, American Institute of Physics Conf. Proc. No. 187, Particles and Fields Series 37, edited by Kenneth J. Heller, (AIP, New York, 1989), p. 1366.

29. T. Yoshida, K. Imai, A. Konaka, S. Makino, A. Masaike, T. Nakano, K. Miyake, T. Nagamine, N. Tamura, F. Takeuchi, R. Takashima, T. Maki, K. Kuroda, A. Michalowicz, D. Grosnick, D. Hill, D. Lopiano, Y. Ohashi, T. Shima, H. Spinka, R. Stanek, D. Underwood, A. Yokosawa, D. Carey, R. Coleman, D. Cossairt, L. Read, F. Luehring, D. Miller, M. Corcoran, F. Nessi-Tedaldi, M. Nessi, G. Phillips, J. Roberts, Q. Zhu, A. de Lesquen, L. van Rossum, F. Lehar, A. Derevschikov, A. Meschanin, V. Solovyanov, Y. Matulenko, S. Nurushev, A. Vasiliev, A. Penzo, G. Salvato, P. Schiavon, A. Villari, A. Zanetti, and G. Pauletta, "Primakoff Polarimeter at Fermilab Polarized Proton/Antiproton Beam Facility," Proceedings of the 8th International Symposium on High Energy Spin Physics, Sept. 10-17, 1988, Minneapolis, Minnesota, American Institute of Physics Conf. Proc. No. 187, Particles and Fields Series 37, edited by Kenneth J. Heller, (AIP, New York, 1989), p. 1360.

30. W. F. Kielhorn, E. Gülmez, J. J. Jarmer and S. I. Penttilä, "Polarization of a Polarized Deuteron Target from NMR Data," Proceedings of the 8th International Symposium on High Energy Spin Physics, Sept. 10-17, 1988, Minneapolis, Minnesota, American Institute of Physics Conf. Proc. No. 187, Particles and Fields Series 37, edited by Kenneth J. Heller, (AIP, New York, 1989), p. 1254.

31. J. C. Peng, D. M. Alde, H. W. Baer, T. A. Carey, G. T. Garvey, A. Klein, C. Lee, M. J. Leitch, J. W. Lillberg, P. L. McGaughey, C. S. Mishra, J. M. Moss, C. N. Brown, W. Cooper, Y. B. Hsiung, R. Guo, D. M. Kaplan, R. L. McCarthy, G. Danner, M. J. Wang, M. Adams, M. L. Barlett, and G. 
W. Hoffmann, "Lepton-Pair Production in Hadron-Nucleus Collisions," in Nuclear and Particle Physics on the Light Cone, Proceedings of the LAMPF Workshop on Nuclear and Particle Physics on the Light Cone, Los Alamos, NM, July 18-22, 1988, edited by M. B. Johnson and L. S. Kisslinger, (World Scientific, Singapore, 1989), p. 65.

32. R. E. Kozack, B. C. Clark, S. Hama, V. K. Mishra, R. L. Mercer, and L. Ray, "Spin-One Kemmer-Duffin-Petiau Equations and Intermediate Energy Deuteron-Nucleus Scattering," Phys. Rev. C 40, 2181 (1989).

33. M. L. Barlett, G. W. Hoffmann, L. Ray, G. Pauletta, K. H. McNaughton, J. F. Amann, K. W. Jones, J. B. McClelland, M. W. McNaughton, R. Fergerson, and D. Lopiano, "Inclusive and Frclusive Quasielastic $\vec{p}+{ }^{2} \mathrm{H}$ Spin Observables at 647 and $800 \mathrm{MeV},{ }^{n}$ Phys. Rev. C 402697 (1989).

34. L. Ray, "Effective Interactions for Nucleon-Nucleus Scattering Above $300 \mathrm{MeV}$," Phys. Rev. C 41, 2816 (1990).

35. M. G. Moshi, C. A. Whitten, Jr., B. Aas, G. J. Igo, D. Lopiano, L. Ray, K. Jones, and M. Gazzaly, "Analyzing Powers for $\vec{p}+{ }^{208} \mathrm{~Pb}$ at Large Momentum Transfers," Phys. Rev. C 41, 1279 (1990).

36. G. W. Hoffmann, M. L. Barlett, D. Ciskowski, G. Pauletta, M. Purcell, L. Ray, J. F. Amann, J. J. Jarmer, K. W. Jones, S. Penttilä, N. Tanaka, M. M. Gazzaly, J. R. Comfort, B. C. Clark, and S. Hama, "Cross Sections, Analyzing Powers, and Spin Rotation Depolarization Observables for $500 \mathrm{MeV}$ Proton Elastic Scattering from ${ }^{12} \mathrm{C}$ and ${ }^{13} \mathrm{C}$," Phys. Rev. C 41, 1651 (1990).

37. W. R. Coker and L. Ray, "Comparison of Dirac and Schrödinger Descriptions of Spin Observables for Proton-Nucleus Elastic Scattering at 650 and $800 \mathrm{MeV}$," Phys. Rev. C 42, 659 (1990).

38. D. M. Kaplan, R. Guo, C. N. Brown, W. E. Cooper, M. J. Wang, T. A. Carey, M. J. Leitch, P. L. McGaughey, C. S. Mishra, J. M. Moss, J. C. Peng, M. L. Barlett, and G. W. Hoffmann, "Test of Scaling of the Massive-Dihadron Cross Section," Phys. Rev. D 41, 2334 (1990).

39. R. E. Chrien, S. Bart, M. May, P. H. Pile, R. J. Sutter, P. Barnes, B. Bassalleck, R. Eisenstein, G. Franklin, R. Grace, D. Marlow, R. Rieder, J. Seydoux, J. Szymanski, W. Wharton, J. Derderian, Y. Civelekoglu, M. Deutsch, J. Prater, C. Chu, R. Hackenburg, E. Hungerford, T. Kishimoto, T. Fukuda, M. Barlett, G. Hoffmann, E. C. Milner and R. L. Stearns, "Search for Radiative Transitions in the Hypernucleus ${ }_{\Lambda}^{10} \mathrm{~B},{ }^{n}$ Phys. Rev. C 41, 1062 (1990).

40. G. Hoffmann, M. Barlett, W. Kielhorn, G. Pauletta, M. Purcell, L. Ray, J. Amann, J. Jarmer, K. Jones, S. Penttilä, N. Tanaka, G. Burleson, J. Faucett, 
M. Gilani, G. Kyle, L. Stevens, T. Mack, K. Mihailidis, D. Dehnhard, T. A vereti, J. Comfort, J. Georgen, J. Tinsley, B. Clark, and S. Hama, "Proton Elastic Scattering from Polarized ${ }^{13} \mathrm{C}$ at $500 \mathrm{MeV}$," PANIC XII, International Conference on Particles and Nuclei, M.I.T., June 25-29, 1990, p. II-10.

41. R. Guo, D. M. Kaplan, D. M. Alde, T. A. Carey, M. J. Leitch, P. L. McGaughey, C. S. Mishra, J. M. Moss, J. C. Peng, C. N. Brown, W. E. Cooper, M. J. Wang, M. L. Barlett, and G. W. Hoffmann, "Improved Limit on Axion Production in 800-GeV Hadronic Showers," Phys. Rev. D 41, 2924 (1990).

42. J. Görgen, J. Comfort, T. Averett, J. DeKorse, B. Franklin, B. Ritchie, J. Tinsley, G. Kyle, B. Berman, G. R. Burleson, K. Cranston, A. Klein, J. A. Faucett, J. Jarmer, J. N. Knudson, S. Penttilä, N. Tanaka, B. Brinkmoeller, D. Dehnhard, Y. F. Fen, B. Breuer, M. A. Khandaker, D. L. Naples, B. S. Flanders, D. Zhang, M. Barlett, G. W. Hoffmann, M. Prucell, and S. Hoibraten, "Asymmetry Measurements for the $\left(\pi^{+}, \pi^{0}\right)$ Reaction on Polarized ${ }^{13} \mathrm{C}$," PARIS 90, 7th International Conference on Polarization Phenomena in Nuclear Physics, Paris, France, 9-13 July, 1990, p. 10F.

43. J. Görgen, J. Comfort, T. Averett, J. DeKorse, B. Franklin, B. Ritchie, J. Tinsley, G. Kyle, B. Berman, G. R. Burleson, K. Cranston, A. Klein, J. A. Faucett, J. Jarmer, J. N. Knudson, S. Penttilä, N. Tanaka, B. Brinkmoeller, D. Dehnhard, Y. F. Fen, B. Breuer, M. A. Khandaker, D. L. Naples, B. S. Flanders, D. Zhang, M. Barlett, G. W. Hoffmann, M. Prucell, and S. Hoibraten, "Polarization Asymmetries for the reaction $\pi^{-} \vec{p} \rightarrow \pi^{0} n$ at $T_{\pi^{-}}$ $=161 \mathrm{MeV}$," PARIS 90, 7th International Conference on Polarization Phenomena in Nuclear Physics, Paris, France, 9-13 July, 1990, p. 1A.

44. Yi-Fen Yen, B. Brinkmoller, S. Chakravarti, D. Dehnhard, S. M. Sterbenz, Yi-Ju Yu, B. Berman, G. R. Burleson, K. Cranston, A. Klein, G. S. Kyle, R. Alarcon, T. Averett, J. R. Comfort, J. Görgen, B. Ritchie, J. M. Tinsley, G. W. Hoffmann, K. Johnson, M. Purcell, H. Ward, A. Williams, J. A. Faucett, S. J. Greene, J. Jarmer, J. A. McGill, C. L. Morris, S. Penttilä, N. Tanaka, H. T. Fortune, E. Insko, R. Ivie, J. M. O'Donnell, D. Smith, S. Hoibraten, M. Khandaker, "Asymmetry Measurements of Pion Elastic Scattering from Polarized ${ }^{13} \mathrm{C}$," PANIC XII, International Conference on Particles and Nuclei, M.I.T., June 25-29, 1990, p. III-65.

45. D. M. Alde, H. W. Baer, T. A. Carey, G. T. Garvey, A. Klein, C. Lee, M. J. Leitch, J. W. Lillberg, P. L. McGaughey, C. S. Mishra, J. M. Moss, J. C. Peng, C. N. Brown, W. E. Cooper, Y. B. Hsiung, M. R. Adams, G. Guo, D. M. Kaplan, R. L. McCarthy, G. Danner, M. J. Wang, M. L. Barlett, and G. W. Hoffmann, "Nuclear Dependence of Dimuon Production at $800 \mathrm{GeV}$;" Phys. Rev. Lett. 64, 2479 (1990). 
46. M. R. Adams, D. M. Alde, H. W. Baer, M. L. Barlett, C. N. Brown, T. A. Carey, W. E. Cooper, G. Danner, G. T. Garvey, R. Guo, Y. B. Hsiung, G. W. Hoffmann, D. M. Kaplan, A. Klein, C. Lee, M. J. Leitch, J. W. Lillberg, R. L. McCarthy, P. L. McGaughey, C. S. Mishra, J. M. Moss, J. C. Peng, and M. J. Wang, "Precision A-Dependence of Dirnucn Production at $800 \mathrm{GeV} / \mathrm{c}$," PANIC XII, International Conference on Particles and Nuclei, M.I.T., June 25-29, 1990, p. VIII-1.

47. Yi-Fen Yen, B. Brinkmoller, D. Dehnhard, S. M. Sterbenz, Y'i-Ju Yu, B. Berman, G. R. Burleson, K. Cranston, A. Klein, G. S. Kyle. R. Alarcon, T. Averett, J. R. Comfort, J. Görgen, B. Ritchie, J. M. Tinsley, G. W. Hoffmann, K. Johnson, M. Purcell, H. Ward, A. Williams, J. A. Faucett, S. J. Greene, J. Jarmer, J. A. McGill, C. L. Morris, S. Penttilä, N. Tanaka, H. T. Fortune, E. Insko, R. Ivie, J. M. O'Donnell, D. Smith, S. Hoibraten, M. Khandaker, S. Chakravarti, "Pion Elastic Scattering from Polarized ${ }^{13} \mathrm{C}$ in the Energy Region of the (3,3) Resonance," PARIS 90, 7th International Conference on Polarization Phenomena in Nuclear Physics, Paris, France, 9-13 July, 1990, p. 12F.

48. E. Sugarbaker, D. Marchlenski, T. N. Taddeucci, L. J. Rybarcyk, J. B. McClelland, T. A. Carey, R. C. Byrd, C. D. Goodman, W. Huang, J. Rapaport, D. Mercer, D. Prout, W. P. Alford, E. Gülmez, C. A. Whitten, and D. Ciskowski, "Isovector Effective Interactions from ${ }^{14} \mathrm{C}(p, n)^{14} \mathrm{~N}$ Studies between 500 and $800 \mathrm{MeV}$," Phys. Rev. Lett. 65, 551 (1990).

49. T. N. Taddeucci, W. P. Alford, M. L. Barlett, R. C. Byrd, T. A. Carey, D. E. Ciskowski, C. C. Foster, C. Gaarde, C. D. Goodman, C. A. Goulding, E. Gülmez, W. Huang, D. J. Horen, J. Larsen, D. Marchlenski, J. B. McClelland, D. Prout, J. Rapaport, L. J. Rybarcyk, W. C. Sailor, E. Sugarbaker, and C. A. Whitten, Jr., "Zero-degree Cross Sections for the ${ }^{7} \mathrm{Li}(p, n)^{7} \mathrm{Be}($ g.s. $+0.43 \mathrm{MeV})$ Reaction in the Energy Range 80-795 MeV," Phys. Rev. C 41, 2548 (1990).

50. T. N. Taddeucci, R. C. Byrd, T. A. Carey, D. E. Ciskowski, C. C. Foster, C. Gaarde, C. D. Goodman, E. Gülmez, W. Huang, D. J. Horen, J. Larsen, D. Marchlenski, J. B. McClelland, D. Prout, J. Rapaport, L. J. Rybarcyk, E. Sugarbaker, I. J. Van Heerden, and C. A. Whitten, Jr., "Gamow-Teller Transition Strengths from the ${ }^{11} \mathrm{~B}(p, n){ }^{11} \mathrm{C}$ Reaction in the Energy Range 160-795 MeV," Phys. Rev. C 42, 935 (1990). 


\subsection{Journal Articles Subrnitted for Publication}

1. W. R. Coker and L. Ray, "The Nuclear Tensor Term in the Relativistic Impulse Approximation for Proton-Nucleus Elastic Scattering," Phys. Rev. C (1990) (in press).

2. S. Shim, B. C. Clark, E. D. Cooper, S. Hama, R. L. Mercer, L. Ray, J. Raynal, and H. S. Sherif, "Comparison of Relativistic and Nonrelativistic Approaches to the Collective Model Treatment of $p+{ }^{40} \mathrm{Ca}$ Inelastic Scattering," Phys. Rev. C (1990) (in press).

3. V. K. Mishra, S. Hama, B. C. Clark, R. E. Kozack, R. L. Mercer, and L. Ray, "Implications of Various Spin-One Relativistic Wave Equations for Intermediate Energy Deuteron-Nucleus Scattering," Phys. Rev. C (1990) (submitted).

4. G. W. Hoffmann, M. L. Barlett, W. Kielhorn, G. Pauletta, M. Purcell, L. Ray, J. F. Amann, J. J. Jarmer, K. W. Jones, S. Penttilä, N. Tanaka, G. Burleson, J. Faucett, M. Gilani, G. Kyle, L. Stevens, T. Mack, D. Mihailidis, T. Averett, J. Comfort, J. Görgen, J. Tinsley, B. C. Clark, S. Hama, and R. L. Mercer, "Polarized Proton Elastic Scattering from Polarized ${ }^{13} \mathrm{C}$," Phys. Rev. Lett. (1990) (submitted).

5. J. J. Görgen, J. R. Comfort, T. Avereti, J. DeKorse, B. Franklin, B. G. Ritchie, J. Tinsley, G. Kyle, B. Berman, G. Burleson, K. Cranston, A. Klein, J. A. Faucett, J. J. Jarmer, J. N. Knudson, S. Penttilä, N. Tanaka, B. Brinkmoller, D. Dehnmard, Y. F. Yen, S. Hoibraten, H. Breuer, B. S. Flanders, M. A. Khandaker, D. L. Naples, D. Zhang, M. L. Barlett, G. W. Hoffmann, and M. Purcell, "Polarization Asymmetries for the reaction $\pi^{-} \vec{p} \rightarrow \pi^{0} n$ at $T_{\pi^{-}}=161 \mathrm{MeV}$," Phys. Rev. D (1990) (in press).

6. N. Tanaka, J. Amann, J. Jarmer, K. Jones, S. Penttilä, M. Barlett, G. Hoffmann, W. Kielhorn, G. Pauletta, M. Purcell, L. Ray, G. Burleson, J. Faucett, M. Gilani, G. Kyle, L. Stevens, T. Mack, K. Mihailidis, D. Dehnhard, T. A verett, J. Comfort, J. Görgen, J. Tinsley, B. Clark, and S. Hama, "Proton Elastic Scattering from Polarized ${ }^{13} \mathrm{C}$ at $500 \mathrm{MeV}$," 9th International Symposium on High Energy Spin Physics, September 10-15, 1990, Physics, Institute, Bonn University, Bonn, Federal Republic of Germany.

7. J. J. Görgen, J. R. Comfort, J. R. Tinsley, T. Averett, J. DeKorse, B. Franklin, B. G. Ritchie, G. Kyle, A. Klein, B. Berman, G. Burleson, K. Cranston, J. A. Faucett, J. J. Jarmer, J. N. Knudson, S. Penttilä, N. Tanaka, B. Brinkmöller, D. Dehnhard, Y. F. Yen, S. Hoibraten, H. Breuer, B. S. Flanders, M. A. Khandaker, D. L. Naples, D. Zhang, M. L. Barlett, G. W. Hoffmann, and M. Purcell, "Analyzing Powers for Pion Charge Exchange on Polarized ${ }^{13} \mathrm{C}$," Phys. Rev. Lett. (1990) (submitted). 
8. D. M. Alde, H. W. Baer, T. A. Carey, G. T. Garvey, A. Klein, C. Lee, M. J. Leitch, J. Lillberg, P. L. McGaughey, C. S. Mishra, J. M. Moss, J. C. Peng, C. N. Brown, W. E. Cooper, Y. B. Hsiung, M. R. Adams, R. Guo, D. M. Kaplan, R. L McCarthy, G. Danner, M. Wang, M. Barlett, and G. W. Hoffmann, "A-Dependence of $J / \psi$ and $\psi^{\prime}$ Production at $800 \mathrm{GeV} / \mathrm{c}$," Phys. Rev. Lett. (1990). (submitted).

9. E. Gülmez, S. Beedoe. A. G. Ling, C. A. Whitten, Jr., M. W. McNaughton, J. R. Santana, D. L. Adams, V. R. Cupps, A. J. Simon, M. L. Barlett, K. H. McNaughton, and P. J. Riley, "Absolute Differential Cross-Section Measurements for Proton-Deuteron Elastic Scattering at 641.3 and $792.7 \mathrm{MeV}$," Phys. Rev. C (1990) (submitted). 


\subsection{Abstracts}

1. G. W. Hoffmann, J. F. Amann, M. L. Barlett, D. Ciskowski, B. C. Clark, J. Comfort, R. W. Fergerson, M. Gazzaly, D. Hill, J. Jarmer, K. W. Jones, R. Mercer, G. Pauletta, S. Penttilä, L. Ray, and N. Tanaka, "Intermediate energy proton + polarized target elastic scattering at LAMPF," Bull. Am. Phys. Soc. 33, 1471 (1988).

2. L. Ray, G. W. Hoffmann, M. L. Barlett, and N. Ottenstein, "Studying relativistic effects in nuclear systems with forward angle proton-nucleus elastic scattering," Bull. Am. Phys. Soc. 33, 1471 (1988).

3. M. L. Barlett, G. W. Hoffmann, L. Ray, G. Pauletta, P. J. Riley, K. H. McNaughton, J. F. Amann, K. W. Jones, J. B. McClelland, M. W. McNaughton, R. Fergerson, and D. Lopiano, "Spin observables for inclusive quasielastic $\vec{p}+{ }^{2} \mathrm{H}$ scattering at intermediate energies," Bull. Am. Phys. Soc. 33, 1471 (1988).

4. G. W. Hoffmann, L. Ray, M. L. Barlett, and N. Ottenstein, "Relativistic Effects in Forward Angle Proton-Nucleus Elastic Scattering," Bull. Am. Phys. Soc. 33, 1079 (1988).

5. M. L. Barlett, G. W. Hoffmann, L. Ray, G. Pauletta, P. J. Riley, K. H. McNaughton, J. F. Amann, K. W. Jones, J. B. McClelland, M. W. McNaughton, R. Fergerson, ara D. Lopiano, "Inclusive and Exclusive Quasielastic $\vec{p}+{ }^{2} \mathrm{H}$ Spin Observables at 647 and $800 \mathrm{MeV}$," Bull. Am. Phys. Soc. 33, 961 (1988).

6. D. Ciskowski, M. L. Barlett, T. A. Carey, J. B. McClelland, L. J. Rybarcyk, T. N. Taddeucci, E. Sugarbaker, D. Marchlenski, C. D. Goodman, W. Huang, E. Gülmez, J. Rapaport, and D. Prout, "Analyzing Power for the ${ }^{15} \mathrm{~N}(p, n){ }^{15} \mathrm{O}$ (g.s.) at $200 \mathrm{MeV}$ and $494 \mathrm{MeV}$," Bull. Am. Phys. Soc. 33, 1583 (1988).

7. T. N. Taddeucci, T. A. Carey, J. B. McClelland, L. J. Rybarcyk, D. E. Ciskowski, M. L. Barlett, E. Sugarbaker, D. Marchlenski, C. D. Goodman, W. Huang, E. Gülmez, J. Rapaport, and D. Prout, "Kinematics, cross sections, and analyzing powers for ${ }^{7} \mathrm{Li}(p, n)$ and ${ }^{12} \mathrm{C}(p, n)$ quasifree scattering at $494 \mathrm{MeV}$," Bull. Am. Phys. Soc. 33, 1597 (1988).

8. L. Ray, "Application of Nonrelativistic Multiple Scattering Theory to DeuteronNucleus Elastic Scattering," Bull. Am. Phys. Soc. 33, 1080 (1988).

9. V K. Mishra, B. C. Clark, S. Hama, R. E. Kozack, R. L. Mercer, and L. Ray, "Relativistic Equations for Deuteron-Nucleus Scattering at Medium Energies," Bull. Am. Phys. Soc. 33, 1080 (1988). 
10. L. Ray and J. R. Shepard, "Spin-Flip Cross Sections for ('harge Exchange Reactions at 500 MeV." Bull. Am. Phys. Soc. 33. Lisi (1988).

11. V. K. Mishra, B. C. Clark, S. Hama, R. E. Kozack, R. L. Mercer, and L. Ray, "Comparison of Relativistic Wave Equations for Deuteron-Nucleus Scattering at Medium Energies," Bull. Am. Phys. Soc. 33, 1607 (1988).

12. V. K. Mishra, B. C. Clark. S. Hama, R. E. Kozack, R. L. Mercer, and L. Ray, "Relativistic Wave Equations for Deuteron-Nucleus Scattering at Medium Energies," Bull. Am. Phys. Soc. 33, 1804 (1988).

13. A. Sethi, D. Cook, M. Franey, M. Gazzaly, E. Heide, N. Hintz, T. Mack, D. Lopiano, S. Nanda, G. Pauletta, and E. Shera, "Inelastic Proton Scattering from Transitional Nuclei and the IBA Model," Bull. Am. Phys. Soc. 33, 1597 (1988).

14. E. Gülmez, S. Beedoe, A. G. Lirg, C. A. Whitten, Jr., M. W. Mc.vaughton, J. R. Santana, M. L. Barlett, K. H. Mcivaughton, P. J. Riley, V. R. Cupps, D. L. Adams, and A. J. Simon, "The Absolute $p-d$ Elastic Scattering Cross Sections at 650 and $800 \mathrm{MeV}, "$ Bull. Am. Phys. Soc. 34. 1140 (1989).

15. S. Shim, B. C. Clark, S. Hama, L. Ray and R. L. Mercer, "The Coupled Channel Calculation for Inelastic Proton-Nucleus Scattering in the Dirac Formalism," Bull. Am. Phys. Soc. 34, 1141 (1989).

16. L. Prout, C. Zafiratos, D. Lind. D. Mercer, M. Braunstein, J. Ullmann, P. Lisowski, G. Morgan, 'T. Taddeucci, L. Rybarcyk, T. Carey, R. Byrd, J. McClelland, R. Jeppesen, W.Amian, E. Sugarbaker, D. Marchlenski, J. Kruzan, P. Kernan, E. Gülmez, C. Whitten, C. Goodman, W. Huang, D. Ciskowski, J. Rapaport, and P. Alford, "Quasi-Free Scattering and Delta Production in $800 \mathrm{MeV}(p, n)$ Reactions," Bull. Am. Phys. Soc. 34, 1142 (1989).

17. G. W. Hoffmann, "Spin Observables for Polarized Proton + Polarized ${ }^{13} \mathrm{C}$ Elastic Scattering at $500 \mathrm{MeV}, "$ Bull. Am. Phys. Soc. 34, 1211 (1989).

18. L. Ray, "Antisymmetrization Correction for Nucleon-Nucleus Elastic Scattering," Bull. Am. Phys. Soc. 34, 1244 (1989).

19. M. J. Wang, H. W. Baer, G. Danner, D. Alde, J. Boissevain, T A. Carey, G. Garvey, J. Kapustinsky, A. Klein, M. J. Leitch, C. Lee, J. W. Lillberg, P. L. McGaughey, C. S. Mishra, J. M. Moss, J. C. Peng, W. E. Sondheim, C. N. Browil, W. E. Cooper, Y. B. Hsiung, K. Luk, M. Adams, R. S. Guo, D. Mí. Kaplan, M. L. Barlett, G. W. H^ffmann, and R. L. McCarthy, "Precision A -dependence of $p+\mathrm{A} \rightarrow \mu^{+} \mu^{-}+\mathrm{X}$ at $800 \mathrm{GeV}, "$ Bull. Am. Phys. Soc. 34, 1804 (1989). 
20. L. Ray, "Nuclear Medium Modifications to the NN Effective $t$ matrix above the Pion Production Threshold," Bull. Am. Phys. Soc. 34, 1822 (1989).

21. D. G. Marchlenski, P. Kernan, J. Kruzan, E. Sugarbaker, R. C. Byrd, T. A. Carey, J. B. McClelland, L. J. Rybarcyk, T. N. Taddeucci, C. D. Goodman, W. Huang, J. Rapaport, D. E. Ciskowski, D. Mercer, D. Prout, E. Gülmez, C. Whitten, and W. P. Alford, "Energy Dependence of Fermi/GT Interactions Via the ${ }^{14} \mathrm{C}(p, n)^{14} \mathrm{~N}$ Reaction at Intermediate Energies," Bull Am. Phys. Soc., 34, 1829 (1989).

22. G. Hoffmann, M. Barlett, W. Kielhorn, G. Pauletta, M. Purcell, L. Ray, J. Amann, J. Jarmer, K. Jones, S. Penttilä, N. Tanaka, G. Burleson, J. Faucett, M. Gilaini, G. Kyle, L. Stevens, T. Mack, D. Mihailidis, T. Avereti, J. Comfort, J. Georgen, J. Tinsley, B. Clark, S. Hama, and R. Mercer, "500 MeV Elastic $\vec{p}+{ }^{13} \overrightarrow{\mathrm{C}}$ Analyzing Power Measurements," Bull. Am. Phys. Soc. 34, $1829(1989)$.

23. S. Shim, B. C. Clark, E. D. Cooper, S. Hama, R. L. Mercer, L. Ray, and J. Raynal, "Comparison of KMT and RIA Results for Collective Model Calculations of Inelastic Proton Scattering from Spherical Targets," Bull. Am. Phys. Soc. 35, 1018 (1990).

24. G. Hoffmann, M. Barlett, W. Kielhorn, G. Pauletta, M. Purcell, L. Ray, J. Amann, J. Jarmer, K. Jones, S. Penttilä, N. Tanaka, G. Burleson, J. Faucett, M. Gilaini, G. Kyle, L. Stevens, T. Mack, D. Mih vilidis, T. Avereti, J. Comfort, J. Georgen, J. Tinsley, B. Clark, S. Hama, R. Mercer, "500 MeV Elastic $\vec{p}+{ }^{13} \overrightarrow{\mathrm{C}}$ Analyzing Power Measurements," Bull. Am. Phys. Soc. 35, 1038 (1990).

25. W. Rory Coker and L. Ray, "Comparison of Dirac and Schrödinger Descriptions of Spin Observables for Proton-Nucleus Elastic Scattering at 650 and $800 \mathrm{MeV}$," Bull. Am. Phys. Soc. 35, 1038 (1990). 

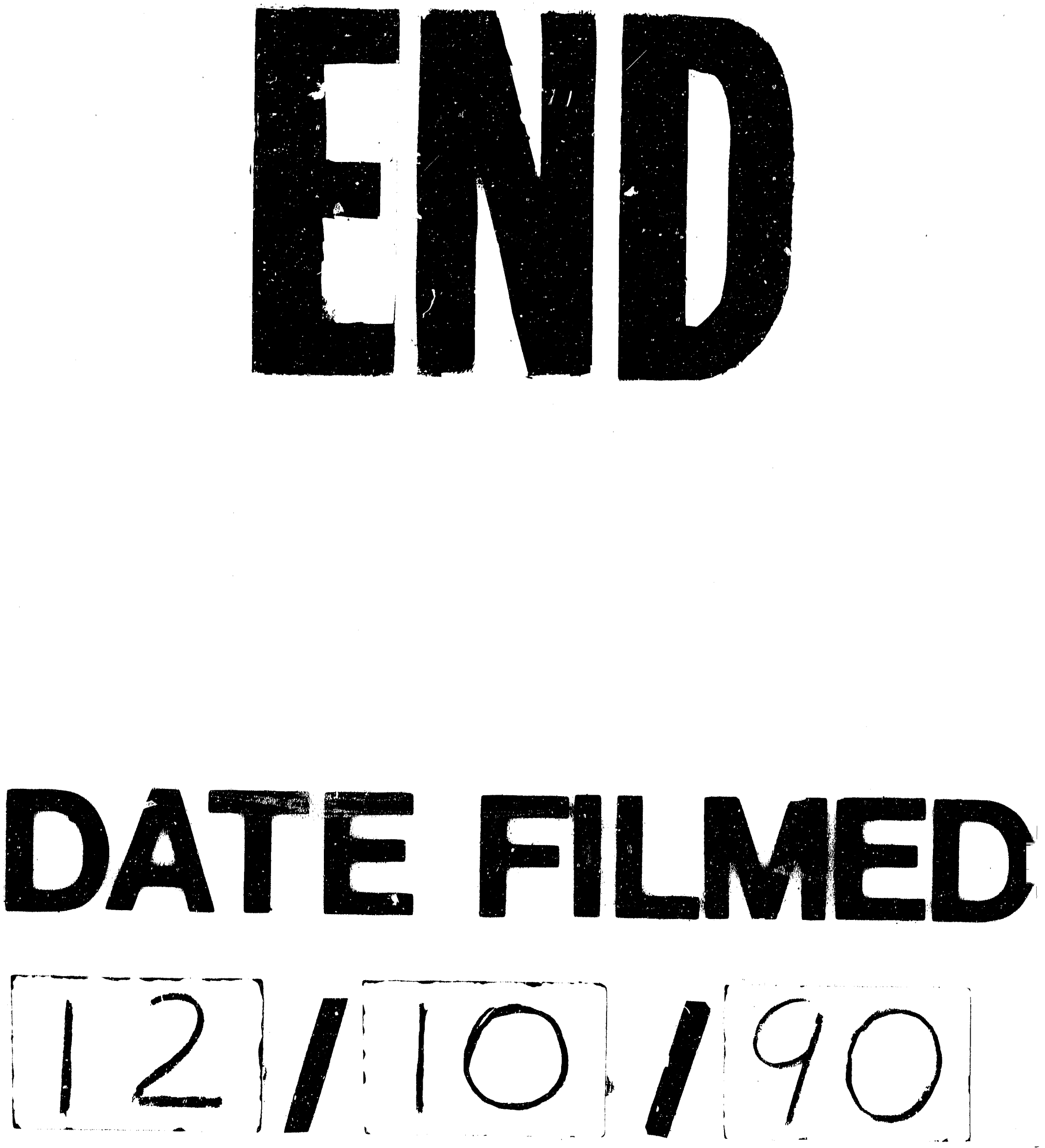
